\title{
Using Concept Mapping to Identify Action Steps for Physical Activity Promotion in Cancer Treatment
}

\author{
Sean J. Fitzpatrick \\ West Virginia University
}

Follow this and additional works at: https://researchrepository.wvu.edu/etd

\section{Recommended Citation}

Fitzpatrick, Sean J., "Using Concept Mapping to Identify Action Steps for Physical Activity Promotion in Cancer Treatment" (2012). Graduate Theses, Dissertations, and Problem Reports. 3582.

https://researchrepository.wvu.edu/etd/3582

This Dissertation is protected by copyright and/or related rights. It has been brought to you by the The Research Repository @ WVU with permission from the rights-holder(s). You are free to use this Dissertation in any way that is permitted by the copyright and related rights legislation that applies to your use. For other uses you must obtain permission from the rights-holder(s) directly, unless additional rights are indicated by a Creative Commons license in the record and/ or on the work itself. This Dissertation has been accepted for inclusion in WVU Graduate Theses, Dissertations, and Problem Reports collection by an authorized administrator of The Research Repository @ WVU.

For more information, please contact researchrepository@mail.wvu.edu. 
Using Concept Mapping to Identify Action Steps for Physical Activity Promotion in Cancer Treatment

Sean J. Fitzpatrick

Dissertation submitted to the College of Physical Activity and Sport Sciences at West Virginia University in partial fulfillment of the requirements for the degree of:

Doctorate in Philosophy: Sport and Exercise Psychology

\author{
Samuel Zizzi, Ed.D., Chair \\ Sean Bulger Ed.D. \\ Reagan Curtis. Ph.D. \\ Kathryn Schmitz, Ph.D., MPH \\ Jack Watson II, Ed.D.
}

Morgantown, WV

April, 162012

Keywords: cancer, exercise, concept-mapping 


\begin{abstract}
Using Concept Mapping to Identify Action Steps for Physical Activity Promotion in Cancer Treatment
\end{abstract}

Sean J. Fitzpatrick

The benefits of exercise during and after cancer treatment represent research areas that have received increased attention throughout the past two decades. Numerous benefits have been observed for cancer survivors who are physically active (Speck, Courneya, Masse, Duval \& Schmitz, 2010). Yet oncologists have been slow to incorporate exercise counseling into practice (Daley et al., 2007). The objective of this study was to gather input from both researchers and oncologists as to what needs to happen for exercise to become part of standard care once treatment ends. The concept mapping process developed by Trochim and Kane (2007) was utilized to realize this goal. A total of 37 unique ideas were produced by participants and were grouped into six-clusters: "Education," "Inclusion of Exercise Professionals," "Changes within the Current Oncology Environment," "Research," "Needed Components of Exercise Programs," and "Patient Focused." Each of these clusters represents an area of concentration where changes are suggested for further promotion of PA for cancer survivors. Participants also rated each idea on its importance and feasibility. The "Education" cluster included many of the ideas that were rated highly on both of these variables and as such may serve as an ideal starting point for increasing cancer survivors' exercise levels. Additionally, results revealed differences between researchers and oncologists with regard to how they ranked the ideas in terms of importance and feasibility. 


\section{ACKNOWLEDGEMENTS}

People always say that it takes a village to raise a child; I suppose this is true though I don't yet have children so I can't speak to the reality of this axiom. However, this project has been my baby of sorts for the past year and I can say without hesitation that it would have never been completed without the help of those I am fortunate enough to have around me.

I want to begin with my committee. Each and every single member of my committee was chosen for a distinct purpose and I could not be more thankful for the help and support of each member.

Dr. Watson: In many ways I approached you for your participation on this project to complete the circle of my higher education experience. Without your influence over the past ten years I would not be here today. You were the first professor I had that challenged me to actually dedicate myself to my studies and without your subtle encouragement years ago there is a betterthan-not chance that I would have continued to skirt by. I owe you a lifetime of thank yous for your inspiration and your belief in me.

Dr. Bulger: As a two-time member of my committee I have come to value your input and approach. I consider myself to be very lucky to have been able to work with you in many different arenas that past few years and I have always admired your consummate professionalism as well as your ability and willingness to take on various roles both small and large. You have provided quite the model for me as I begin my professional career.

Dr. Curtis: I like to joke that I have a cognate in the "Curtis Research Methods" as I believe I have taken every single one of your research courses. You have helped me shape my research view and approach. More specifically you opened my eyes to Concept Mapping years ago. Your willingness to offer an independent study opportunity to teach me this method two summers ago served as the incubator for this project.

Dr. Schmitz: One of the most important events that led to me pursuing this project was the phone call you agreed to take a year and a half ago. I contacted you completely out of the blue and you could have very easily ignored my request; instead you not only agreed to chat, you spoke with me for an hour, offered your invaluable insight and encouraged me to pursue this study. This altruistic act on your part is topped only by your willingness to serve on this committee and your continued support and guidance throughout the project. I can only hope that one day I will be able to emulate your kindness and compassion to a young researcher such as myself. I hope that one day we can actually meet!

And this leaves Dr. Z. For me to properly put my thoughts into words would require space that I know the ETD office would never commission and would fly counter to your continued push for parsimony. You've been my advisor for literally a decade. Throughout this 
time you've served countless roles in my life and have done so with a grace that cannot be overstated. You've always known when to push, when to let me be, and when to call me out. I have disagreed with a number of your suggestions and decisions; yet in each and every single one of these instances you proved to be right. I cannot fathom how I could have made it through the past few years without your guidance, not just in terms of school but life in general. You have taught me more than you will ever know and I am continually amazed at how often I notice your influence on my life.

To my classmates at WVU, though I feel a need to thank pretty much every one of you individually, I will just say that I feel very fortunate to have been able to learn from all of you and share so many memories with you. West Virginia will always be home to me in a special way.

Lastly, to Colleen: I've only had one sister but I can't imagine that there could ever be a better one. Throughout my whole life I have always looked up to you. The sacrifices you made when you moved back home allowed me to pursue my dreams and I will forever be indebted to you in a way that I doubt you'll ever understand. Your dedication to mom and dad is the absolute essence of love. You are, and always will be, my best friend. 


\section{DEDICATION}

This, as is everything I do, is for my mom and dad. Every day I miss both of you. I will always remember your pride for Colleen when she received her Ph.D. and I can only image the scene you both would have created when your second child receives his as well. 
Table of Contents $\quad$ pg\#

$\begin{array}{lr}\text { Introduction } & 1\end{array}$

Methods

Participant Recruitment - Generating the Ideas 6

$\begin{array}{ll}\text { Generating the Ideas } & 7\end{array}$

Participant Recruitment - Structuring the Statements 9

$\begin{array}{ll}\text { Structuring the Statements } & 10\end{array}$

Concept Mapping Analysis $\quad 12$

Results

Demographics

Brainstorming 13

$\begin{array}{ll}\text { Structuring } & 14\end{array}$

Brainstorming \& Structuring the Statements 14

Comparisons between Oncologists and Researchers 16

Discussion

$\begin{array}{ll}\text { Major Findings } & 17\end{array}$

Discrepancy Data between Oncologists and Researchers 22

Limitations $\quad 24$

$\begin{array}{ll}\text { Future Directions } & 27\end{array}$

$\begin{array}{lr}\text { References } & 29\end{array}$

Table 1: Brainstorming Demographics $\quad 35$

Table 2: Structuring Demographics $\quad 36$

Table 3: List of Statements $\quad 37$

Table 4: Statements by Cluster $\quad 39$

Figure 1: Recruitment flow chart for the brainstorming process $\quad \mathbf{4 1}$

Figure 2: Recruitment flow chart for the structuring process 42

Figure 3: Point map of the statement data 43

Figure 4: Six cluster solution cluster map $\quad \mathbf{4 4}$

Figure 5: Four cluster solution cluster map $\quad 45$

Figure 6: Five cluster solution map $\quad 46$

$\begin{array}{ll}\text { Figure 7: Seven cluster solution map } & 47\end{array}$

$\begin{array}{ll}\text { Figure 8: Eight cluster solution map } & 48\end{array}$

Figure 9: Pattern-match display depicting importance and
feasibility ratings of the six clusters

Figure 10: Cluster rating map for importance $\quad 50$

Figure 11: Cluster rating map for feasibility $\quad 51$

Figure 12: Go-zone display depicting the average rating of
every statement on importance and feasibility 
Figure 13: Pattern match display comparing cluster ratings on importance between researchers and oncologists

Figure 14: Pattern match display comparing cluster ratings on feasibility between researchers and oncologists

Figure 15: Go-zone display comparing oncologists’ and researchers' ratings for importance

Figure 16: Go-zone display comparing oncologists’ and researchers' ratings for feasibility

56

Appendix A: Brainstorming Demographic Survey - Researchers 57

Appendix B: Brainstorming Demographic Survey - Oncologists 58

Appendix C: Review of Literature $\quad \mathbf{6 0}$

Cancer: A Brief Primer $\quad 60$

Physical Activity during Treatment $\quad 62$

Selected controlled trials with multiple outcomes $\quad 64$

Physical Activity after Treatment $\quad 70$

Selected controlled trials with multiple outcomes $\quad 72$

Physical Activity Promotion with Cancer Patients 84

Appalachian Health Disparities $\quad 90$

The Concept Mapping Process: Examples from Various

Fields of Public Health $\quad 92$

Conclusions and Future Recommendations 103

$\begin{array}{ll}\text { References } & 105\end{array}$ 
Using Concept Mapping to Identify Action Steps for Physical Activity Promotion in Cancer

Treatment

\section{Introduction}

Throughout the past few decades vast strides have been made in cancer treatment, detection, and prevention. However, there is still much to be done, and cancer, in many ways, is still a disease that is not well understood. The advancements in detection and treatment have led to a drastic increase in survival rates. During the last measured time period (1990-2006) cancer death rates in the U.S. decreased $21 \%$ for men and $12.3 \%$ for women, which equates to over $3 / 4$ of a million avoided deaths. These numbers are promising for the 1.5 million Americans that were estimated to be diagnosed with cancer in 2010 (Jemel, Siegel, Xu, \& Ward, 2010). With the advances in treatment and early detection, many of these individuals will live longer after their diagnosis than individuals who were diagnosed as little as five years ago. Currently it is estimated that there are greater than 12 million cancer survivors in the U.S. (Center for Disease Control, 2010). For these reasons, this number will continue to climb each year, with every breakthrough or advancement leading to sharper increases. Due to the increased number of cancer survivors, there have been calls for an increased focus on the unique needs and challenges of this population (Meadows et al., 1998).

Though cancer treatment has become more effective and more efficient, there are still numerous side-effects from the various treatment forms, many of which remain after treatment has ceased. Cancer and its treatment often cause fatigue and lowers patients' quality of life (QOL) and overall physical functioning. In addition, some cancer patients experience mental health issues such as depression. Cancer survivors are also at risk for secondary cancers and have elevated risk levels for other co-morbidities such as heart disease (Demark-Wahnefried, 
Aziz, Rowland, \& Pinto, 2005). A variety of interventions have been examined to combat these issues. Physical activity (PA) represents one intervention that has received increased attention throughout the past two decades.

The effects of PA have been studied both with cancer survivors undergoing treatment and survivors who have concluded their treatment. Systematic reviews of literature have found that during treatment PA is safe and tolerable for patients and often has positive effects on fitness levels (Schmitz et al., 2010; Speck, Courneya, Masse, Duval, \& Schmitz, 2010). After treatment, these reviews found that PA has been shown to benefit fitness levels, QOL, fatigue, general symptoms, as well as survivors' body image. The individual studies in this line of inquiry have examined numerous variables. Fatigue for instance, which is a common and often debilitating side-effect of treatment, has been shown to be inversely related with the PA levels of survivors undergoing treatment; this effect has been observed among patients with a variety of cancers (e.g. Adamsen et al., 2009; Segal et al., 2009). In a study of breast cancer patients, both affect and QOL increased for women participating in exercise, and these gains remained evident six months after the exercise intervention (Mutire et al., 2007). With survivors who have finished treatment, QOL improvements have been observed for those who are more active (e.g. Daley et al., 2007; Milne, Wallman, Gordon \& Courneya, 2008). Large observational studies have also shown that survivors who possess higher levels of post-diagnosis PA have higher rates of survivorship and have decreased levels of cancer recurrences (e.g. Holick et al., 2008; Holmes, Chen, Feshanich, Kroenke, \& Colditz, 2005; Sternfeld et al., 2009). Though continued investigation is needed, PA has been shown to be beneficial for numerous outcomes for cancer survivors. 
Unfortunately, most cancer survivors experience a significant decrease in activity levels when they are diagnosed (e.g. Courneya \& Friedenreich, 1997 a \& b; Jones \& Courneya, 2002a). What is promising though is that when oncologists suggest exercise to their patients, their patients often listen and substantially increase their activity levels compared to patients of oncologists who do not discuss exercise or PA (Jones \& Courneya, 2002b; Jones, Courneya, Fairy, \& Mackey, 2004). Despite this encouraging evidence, studies have shown that a large proportion of oncologists are not regularly promoting PA. For instance, Jones, Courneya, Peddle, and Mackey (2005) found that among a sample of 281 practicing Canadian oncologists, less than 30\% were recommending PA to more than two-thirds of their patients. Similarly, in a study of oncologists and cancer surgeons from the United Kingdom $(n=102)$, less than half (44.1\%) reported that they routinely provided advice to their patients about being active (Daley, Bowden, Rea, Billingham \& Carmicheal, 2008). In the Canadian study (Jones et al., 2005), the majority of the oncologists believed that exercise was beneficial (62\%), important (55.8\%) and safe (63.1\%). Similar results have been found in other studies, for instance, Peeters and colleagues (2009) observed that among the oncologists surveyed $(n=18)$, most $(72 \%)$ believed sufficient evidence existed to confidently suggest that their patients exercise to gain physical benefits. When the patients themselves are examined, it has been found that patients are open to receiving information on increasing their PA levels (e.g. Jones \& Courneya, 2002b; Rogers et al., 2008). It seems that despite the fact that studies have shown PA to be beneficial for cancer patients and many cancer patients are open to the promotion of PA, oncologists do not widely promote exercise.

Numerous behavioral factors, such as tobacco use (e.g. Boffetta, Hecht, Gray, Gupta, \& Straif, 2008) and inactivity (e.g. Lynch, 2010) are related to elevated cancer risks. Research has 
shown that primary care providers can positively influence the health behaviors of their patients and studies have identified the most effective techniques to do so, yet most physicians do not spend much time counseling their patients on these matters. For example, even though smoking cessation programs have been shown to be effective, a recent study of health care professionals found that only $44.7 \%$ of the 437 primary care physicians surveyed provided their patients with referrals to a smoking cessation program and only 54.5\% provided any smoking cessation materials (Tong, Strouse, Hall, Kovac, \& Schroeder, 2010). Other studies have been published that provide evidence-based guidelines concerning primary care counseling for chronic conditions (e.g. smoking and alcohol use). An example of such recommendations is a recent piece by Battersby and colleagues (2010), which reviewed twelve principles for implementing self-management support for chronic conditions. Among these principles were the suggestions to link patients with evidence-based community programs and to further promote multifaceted interventions, suggestions that as the before mentioned study highlighted (Tong et al., 2010), are not being followed. To address this gap between research and practice, Graham, Kerner, Quinlan, Vinson and Best (2008) used concept mapping to investigate how to better integrate research findings / recommendations into medical practice.

Graham and colleagues (2008) recruited researchers and physicians to take part in a concept mapping study to identify strategies to bring research and practice closer together within the domain of cancer prevention. Participants (researchers and primary care physicians) responded to the following prompt: "One thing that we are doing or could do in our setting that would more effectively integrate research with practice is” (p. 242). The statements collected (n = 293) were reduced to 87 statements which stakeholders then grouped and rated both on feasibility and importance. The statements were grouped into 10 clusters, including but not 
limited to: “respond to stakeholder priorities,” “implement innovative professional training,” and “ensure quality and accountability.” These 10 groupings were then used to develop a five-factor meta-model to aid researchers and physicians in integrating research about counseling patients on health behaviors into practice. As this study highlights, concept mapping can be used to gather input from researchers and physicians to begin to better understand how these two groups can work together.

Researchers have found PA to be effective for various outcomes, many oncologists realize this, yet it seems few are actively promoting PA. Concept mapping, just as in the above example (Graham et al., 2008), may represent a way for these two groups to come together to work on this gap between research and practice. The purpose of this study was to gather opinions of researchers and oncologists to better understand what steps are needed to make the promotion of PA a standard part of cancer care.

\section{Methods}

The concept mapping methodology chosen for this study is a systematic process that consists of four preparatory and data collection/analysis steps: preparing for concept mapping, generating the ideas, structuring the statements and concept mapping analysis (Kane \& Trochim, 2007). The purpose of concept mapping is to gather input from stakeholders regarding a certain issue or problem. Through an integration of qualitative (i.e. brainstorming) and quantitative (i.e. multidimensional scaling) methods, visual maps are created to provide insight and direction. A more thorough explanation of the concept mapping process can be found elsewhere (e.g. Kane \& Trochim, 2007 \& Trochim, 1989). 


\section{Participant Recruitment - Generating the Ideas}

The goal of recruitment was to recruit 20 oncologists and 20 researchers to complete the participatory phases of the project. Previous studies using concept mapping within various health promotion contexts had similar numbers of participants (e.g. Anderson et al., 2006, $n=37$;

Burke et al., 2005, $n=37$; Stillman, Hoang, Linton, Ritthiphakdee, \& Trochim, 2008, $n=31$; \& Trochim, Milstein, Wood, Jackson, \& Pressler, 2004, $n=25$ ). In addition, the study that most closely resembles the current study, Graham et al.’s (2008) work examining a similar gap between research and practice in cancer prevention, had 36 individuals complete the structuring phase (the last participatory step).

To be eligible to participate as a researcher, an individual must have been first author on a paper regarding exercise and cancer treatment within the past five years. To compile a list of potential participants, a search of articles was completed using the MEDLINE database. The following key words were used as search terms for studies published since 2006: "exercise or physical activity” and “cancer”. Reference lists of recent reviews and other relevant articles were also examined. Only information from researchers from North America was collected. Lead authors' email addresses, if not provided, were then attained through an internet search of the researcher's name and institution. Thirty-six researches were identified through these means. Researchers were contacted through email for participation.

To be eligible for participation in the study as an oncologist, individuals must have been currently practicing oncology. Initially, it was decided that only oncologists from within Appalachia would be contacted for potential participation. A list of 52 cancer centers throughout the Appalachian region was created. The Association of Community Cancer Center's (ACCC) website was used to compile this list (ACCC, 2011). The ACCC is an organization of 
community cancer centers throughout the United States. The organization's website lists its membership of cancer centers by state. Each participating cancer center has contact information; in most cases this individual is the director of the cancer center. Contact information (names, addresses and phone numbers) for the 52 cancer centers that resided within the 13 states that make up the Appalachia was collected.

To recruit researchers, emails were sent to solicit participation. Emails were sent out through the Survey Monkey online software. Researchers were contacted up to three times each to participate. To recruit oncologists, the contact individuals at the identified cancer centers were called and were asked to send out an email to oncologists on the researcher's behalf. Each of these individuals was called up to three times each. A small incentive was used to recruit oncologists; a \$10 donation to the oncologists’ cancer center was made for each participating oncologist. If phone contact was not successful, a mailing was sent to the contact individual that included hard copies of the project materials. The contact individual was instructed to pass out these materials to oncologists. Addressed and stamped return envelops were included; participants were also made aware that they could fax the materials back or could request to participate online.

Twenty-eight researchers and twelve oncologists were successfully recruited to participate in the brainstorming via the recruitment means described above. See Figure 1 for a breakdown of the recruitment and participation for both of these groups during the brainstorming phase.

\section{Generating the Ideas}

The following prompt was used to stimulate statements from participants: "One thing that needs to happen for exercise to become part of standard cancer care once treatment ends is...” 
The conclusion of treatment was chosen as the time point for this study for a number of reasons. Chief among these reasons was the definable nature of this time point for a cancer survivor. If the beginning of treatment was chosen, participants may have viewed different occurrences as the "start" of treatment; surgery, diagnosis, adjunct therapy etcetera. Also, while variability still very much exists between survivors at this chosen time point, there is less variability than there would be with time points during treatments. In addition to providing the prompt, participants were told to provide as many statements that complete this sentence as they could. Participants were also able to see the previously provided statements, which is done to make the online data collection similar to in-person collections methods where individuals brainstorm ideas together.

In addition to providing ideas during this phase, participants provided demographic information (see Appendixes A \& B for the demographic surveys used). Individuals were emailed a link which led to an online survey to collect demographic information. Though the demographic information collected from oncologists and researchers was similar, there was a separate survey for each group. Both groups were asked basic demographic questions: gender, age, and experience. Both groups were also asked which type of cancer they had the most experience working with. Physical activity (PA) habits were collected from researchers and oncologists; participants were asked how many days a week they partook in at least 30 minutes of moderate activity as well as how many days a week they participated in at least 20 minutes of vigorous activity. All participants were also asked if they believed that exercise should be part of standard care via a six-point Likert-type item (“1 = Strongly Disagree”, “6 = Strongly Agree”); additionally, a text box was provided and participants were asked to provide a brief response to this answer. Researchers were asked one additional question: "What is the approximate number of physical activity and cancer studies that you have been involved with throughout your 
career?” Oncologists were asked if they had ever taken part in a research study regarding exercise and cancer treatment. They were also asked to provide the breakdown of how they spent their professional time between research and practice. Two other professional questions were asked of oncologists: the type of oncology they practiced (medical, survival, radiation, other) as well as their primary setting (community based cancer center or academic cancer center). At the end each demographic survey a link was provided to the study’s Concept Systems website (Concept Systems, Inc Ithaca, NY) where participants participated in the brainstorming exercise.

\section{Participant Recruitment - Structuring the Statements}

Due to the trouble recruiting oncologists for the brainstorming phase, recruitment was broadened for the second phase of participation. All of the participating oncologists from the brainstorming phase $(n=12)$ were contacted to participate in the second phase. Additionally, state and national level oncology societies $(n=11)$ were contacted to forward a recruitment email to oncologists in their respective society. One of these societies, the Association of Northern California Oncologists, declined to forward an email, but provided a directory of its oncologists. Three hundred and ten working emails were compiled from the directory. Each member was contacted up to three times to participate; agreeing oncologists were reminded up to three times each to participate in one-week intervals. Lastly, oncologists were recruited through the National Cancer Institute’s (NCI) Community Cancer Oncology Programs (CCOPs). This program “... was created in 1983 as a national mechanism for community-based physicians to partner with academic investigators. The primary goal was to accelerate implementation of NCI clinical trials for cancer prevention, control and treatment.” (NCI, 2012). The NCI’s website lists contact information for all 63 CCOPs; emails were sent to the contact individuals requesting that they forward an email to oncologists. Twenty-four of the CCOPs agreed to this request. 
With regard to researchers, all of the participating researchers from the previous phase were contacted for participation. Additionally, non-participating researchers were contacted as researchers declined to participate or did not respond to email requests. Each researcher was emailed directly up to three times. Additionally, researchers were contacted by Dr. Katherine Schmitz via email to further increase participation; the researchers targeted in this email solicitation included both previously contacted researchers as well as new researchers.

Figure 2 provides a breakdown of recruiting for the structuring phase. Five of the oncologists who participated in the first phase also took place in the second phase; fifteen oncologists participated from the CCOPs; 16 oncologists participated from the Association of Northern California Oncologists; lastly, two oncologists were forwarded a message from researchers who participated in the study, for a total of 38 participating oncologists. A total of 20 researchers participated in this step as well.

\section{Structuring the Statements}

When the brainstorming stage ended, the list of statements was reduced and finalized. This data reduction was accomplished through a modified qualitative content analysis; like statements were combined, irrelevant statements were deleted, and many of the statements were edited to ensure that they were easier to understand. Two researchers (SF \& SZ) independently completed this process and then met, compared their results, and collaborated when differences were found.

Once the statements were finalized, participants were contacted via the recruitment means described previously. Participants were provided with a username and password so that they could log-in to the study’s website to complete the remaining participatory tasks. Upon logging-in participants were provided with a brief overview of what they were being asked to do 
and were provided links to the three steps of this stage. The first task was to answer 5 demographic questions: their professional role (oncologist or researcher), their professional experience, the cancer type they had the most experience working with, as well as their moderate and vigorous physical activity habits (these PA questions were identical to the PA questions from the Brainstorming phase).

The second task was to group the statements. Participants were informed to: "Combine statements into groups in a way that makes sense to you.” Participants were notified that there were four restrictions: 1) “all the statements cannot be placed into a single pile,” 2) "all the statements cannot be put into their own separate group (though there can be groups of just one statement),” 3) “each statement can only be placed into one group,” and 4) "there should not be a miscellaneous group.” Participants accomplished the grouping of statements through the Concept Systems website’s interface (Concept Systems, Inc Ithaca, NY).

The third and final step was to rate each statement. Each statement was rated on two variables: importance and feasibility. Both variables were assessed with four point Likert-type scales. For importance, participants were instructed to: "Rate each statement based on its importance in making exercise part of standard cancer care once treatment has ended $(1=$ not at all important, 4 = very important).” Feasibility was rated on a similar scale $(1=$ not at all feasible, 4 = very feasible). Participants were asked to "Rate each statement on the feasibility that it can be realized within the next five years.” The rating step took place after the sorting step so that participants were not influenced on how they created groups based on the rating data (Kane \& Trochim, 2007).

Overall, a total of sixty four individuals agreed to participate. Six of these individuals did not begin any of the three participant steps of this phase even after multiple email reminders to 
do so. A total of 50 (32 oncologists and 18 researchers) individuals successfully completed the sorting step, while 56 individuals (20 researchers and 36 oncologists) completed the rating tasks for importance and feasibility respectively.

\section{Concept Mapping Analysis}

Concept mapping software (The Concept System, Ithaca, NY) was used to construct the various maps and to analyze the data. Specifically, a similarity matrix was created that represented the similarity of participants' sorting data. Next, multidimensional scaling was used to produce a two-dimensional solution which placed each statement onto an x, y graph.

Statements were assigned $\mathrm{x}$ and y coordinates based on their relationship (mathematical similarity) to other statements. Statements that were often grouped together were placed closer together and statements that were rarely if ever placed together were placed further apart. At this step, a stress value is calculated. The stress value is a diagnostic statistic used in multidimensional scaling that measures the degree to which the map is different from the sorting data provided by participants. The lower the stress value, the more representative the map is. For most multidimensional uses, a stress score below 0.10 is sought; however, due to the uniqueness of the participatory nature of concept mapping the value is often higher (Kane \& Trochim, 2007). In a meta-analysis of concept mapping studies, the average stress score equaled 0.285 (Trochim, 1993). Lastly, hierarchal cluster analysis was used to group the statements into groups. Visually, the results of this analysis are seen by connecting the statements ( $\mathrm{x}, \mathrm{y}$ coordinates) that are grouped together to form polygonal shapes. The final number of clusters was decided upon by two researchers (SF \& SZ). 


\section{Results}

\section{Demographics}

Brainstorming. A total of 26 researchers participated in the brainstorming task of this project. There were three men and 21 women among these individuals (2 participants did not indicate their gender). The average age of the researchers was 45.71 years $(\mathrm{SD}=8.07)$ and these individuals had an average professional career length of 8.42 years $(\mathrm{SD}=4.87)$ and had been involved with just under 10 research studies regarding exercise and cancer $($ mean $=9.96, \mathrm{SD}=$ 11.35). With regard to their physical activity habits, these individuals participated in $4.65(\mathrm{SD}=$ 2.04) and $3.29(\mathrm{SD}=2.01)$ days of moderate and vigorous physical activity each week respectively. As would be expected, this group agreed that with the following statement: "Do you agree that exercise should be part of standard cancer care?” rating their agreement as a 5.65 (SD = 0.65) out of six (“1 = Strongly Disagree”, “6 = Strongly Agree”).

Among the 12 participating oncologists, there were nine males and two females (one participant did not answer this item). The majority of these physicians practiced oncology within a community cancer center $(n=10)$ and medical oncology was the most represented branch of oncology $(n=7)$. The average career length for these participants was $17.17(\mathrm{SD}=12.92)$ years. In terms of their own physical activity habits, the participating oncologists participated in 3.60 $(\mathrm{SD}=1.90)$ days of moderate activity and $2.27(\mathrm{SD}=2.05)$ days of vigorous activity. Lastly, in terms of their level of agreement that exercise should be part of standard cancer care, the oncologists on average, rated their agreement much lower than the researchers: 4.45 (SD = 1.63; $t(31)=3.44, p=0.002, d=1.08$ ) out of six (see Table 1 for a breakdown of demographic information for both oncologists and researchers during brainstorming). 
Structuring. A total of 58 individuals participated in the sorting and rating steps of the concept mapping process. Twenty of these individuals were researchers. Among this group, the average career length was $16.50(\mathrm{SD}=9.26)$ years. Most $(n=12)$ of the individuals identified breast cancer and the cancer type they had the most experience researching. In terms of days per week that these participants were active, they achieved 4.95 (1.28) and 3.15 (1.46) days of moderate and vigorous physical activity respectively.

The 38 oncologists had an average career length of $21.11(\mathrm{SD}=11.54)$ years and again breast cancer was the most commonly identified cancer type $(n=24)$ that the participants worked with. These oncologists participated in approximately three days of both moderate and vigorous physical activity each week (mean $=3.00, \mathrm{SD}=1.90$ for moderate activity and mean $3.05, \mathrm{SD}=2.08$ for vigorous activity). Table 2 provides a comparison of the demographics for these two groups during the structuring phase of the project.

\section{Brainstorming \& Structuring the Statements}

The 38 participants who participated in the brainstorming phase provided 75 total statements. Reduction of these statements yielded 37 unique ideas (Table 3). These 37 statements were then used for the sorting and rating tasks of the structuring phase. Fifty of the 58 participants in the second phase completed the sorting task. Figure 3 provides the results of the sorting task in the form of a Point Map. This map can be considered representative of the data provided by the participants as its stress value is 0.218 .

A six cluster map was chosen as the final cluster solution for the data (Table 4 \& Figure 4). The six clusters created were: 1 "Education” (ex statements: "Educate the cancer team about the benefits of exercise" and "Educate oncologists about the benefits of exercise”), 2 "Inclusion of Exercise Professionals” (ex statements: “Develop connections between healthcare providers 
and community-based exercise professionals" and "Include exercise specialists on the cancer team”), 3 “Changes within the Current Oncology Environment” (ex statements: “Develop systems-oriented intervention approaches that rely less on the oncologists and more on support staff,” and “Prioritize recovery issues”) , 4 "Research” (ex statements: “Conduct larger, better designed studies to confirm current findings," and "Produce more evidence regarding the exact type, dose and timing of exercise needed”), 5 “Needed Components of Exercise Programs” (ex statements: "Provide cost-free exercise programs," and "Develop exercise centers for cancer survivors”) and 6 "Patient Focused ” (ex statements: “Develop effective activity interventions that don't require patients to go to clinic-based facilities" and "Look at the LIVESTRONG at YMCA model of exercise”).

A six cluster model was decided upon for a few reasons. At seven clusters, there were two similar clusters that dealt with programmatic issues of starting exercise programs for cancer patients (these two clusters combined to form cluster 5 "Needed Components of Exercise Programs” in the six cluster model). At five clusters, two distinct cluster came together (clusters 2 “Inclusion of Exercise Professionals” \& 3 “Changes within the Current Oncology Environment” in the six cluster solution) to form a broad category that lacked coherency. Figures 5, 6, 7, and 8 provide depictions of four, five, seven and eight cluster solutions respectively.

Based on the average rating of statements within each cluster, Cluster 4, "Research,” was rated as the most important (3.35) and Cluster 2, "Inclusion of Exercise Professionals,” was rated as the least important (2.8). Ratings for feasibility placed Cluster 1, “Education,” as the most feasible (3.45) and again Cluster 2, "Inclusion of Exercise Professionals,” was rated at the bottom (2.54). Figure 9 provides a pattern match ladder which shows the ranking for clusters on 
both importance and feasibility; additionally cluster rating maps visually highlight the rating data for the clusters (Figures 10 \& 11).

Taken individually, statements from Clusters 1 \& 4, “Education,” and "Research” were among the highest rated statements for both importance and feasibility. Go-zone displays provide a visual depiction of statement ratings and can be used to identify statements that are considered to be "actionable" as they are rated highly on both importance and feasibility (Figure 12). A number of statements were rated as both highly important and feasible, including statement 15 “Educate patients about the benefits of exercise,” statement 3 “Educate the cancer care team about the benefits of exercise," and statement 27 "Report research data from successful exercise oncology programs.” Overall the correlation between the importance and feasibility ratings of the statements was 0.42 which suggest that the ratings were moderatley related to one another.

\section{Comparisons between oncologists and researchers}

A number of maps and displays can be used to identify differences in the way the statements were conceptualized between the two groups of participants, oncologists and researchers. At the cluster level, With regard to importance, both groups viewed the clusters similarly as the ranking of clusters matched up closely, though the researchers tended to rate clusters higher (Figure 13). The rating data from the feasibility ratings show a number of differences between the two groups (Figure 14). Oncologists viewed Cluster 1 "Education” as the most feasible cluster, followed by Cluster 3 "Changes within the Current Oncology Environment”, Cluster 6 "Patient Focused”, and Cluster 4 "Research.” Conversely, researchers viewed Cluster 4 "Research” as the most feasible, followed by Cluster 1, 6, and 3 "Education," “Patient Focused” and “Changes within the Current Oncology Environment.” 
The individual statements can also be compared on their ratings between the two groups, using the go-zone displays (see Figure 15 and 16). For both feasibility and importance, group comparison go-zones highlight that there are a number of statements that both groups rated highly, however, there are differences between both groups among both ratings. For example, in terms of importance the oncologists viewed statements 6 \& 7 "Promote home-based exercise programs” and "Provide cost-free exercise programs" as more important than average, the researchers did not. Conversely, the researchers rated the following statements as more important than average whereas the oncologists did not: statement 5 "Train members of the cancer care team on how to properly promote exercise" and statement 22 "Staff exercise programs with trainers who have certifications to work with cancer survivors.” The feasibility data show disagreement as well; examples include the researchers’ view of statement 9 “Confirm the various mechanisms (e.g. biological) that are involved with the benefits of exercise for cancer survivors," which the group believed was more feasible than the average statement which was not a view shared among the oncologists. Two examples of the opposite disagreement are statements 18 and 33 “Oncologists need to promote exercise” and "Have therapists help patients start exercise programs.” Both groups ranked the items similarly on both feasibility and importance, as the correlation values between the two groups ratings equaled $r=0.87$ and $r=$ 0.82 respectively for the two variables.

\section{Discussion}

\section{Major Findings}

The data, supplied by oncologists $(n=32)$ and researchers $(n=18)$, were organized into a six-cluster map made up of the following clusters: "Education," "Inclusion of Exercise Professionals,” “Changes within the Current Oncology Environment,” "Research,” "Needed 
Components of Exercise Programs,” and "Patient Focused.” Each of these clusters represents an area of focus that needs attention to further the promotion of physical activity (PA) within the cancer treatment setting. Aside from the formation of the clusters via the sorting data of participants, participants in the second phase of the study also identified actionable steps that can be prioritized as impactful directions to pursue.

The "Education" cluster represents one of these directions as the statements that make up this cluster were rated highly both on their importance in making exercise part of standard cancer care once treatment has ended and their feasibility of happening within the next five years. Three statements in particular within this group were rated highly on both variables by participants: “Educate patients about the benefits of exercise," "Educate the cancer care team about the benefits of exercise," and "Educate oncologists about the benefits of exercise.” These statements and the "Education" cluster as a whole, represent a potential starting point for increasing PA promotion. Among the three groups identified in the statements, patients, oncologists, and the cancer team, patients were identified as the most important and most feasible group to educate. Steps are currently being taken to further the education of this population with regard to PA. Organizations such as the American Cancer Society and others provide information regarding exercise for cancer patients on their websites and/or through brochures and pamphlets (American Cancer Society, 2011). There has also been work that has investigated patients’ beliefs about exercise. It has been found that patients believe exercise to be beneficial but perhaps one of the explanations for this populations' low level of PA is that they do not necessarily know what to do (Peeters et al., 2009). This lack of understanding of what exercises to do, how long to do them, and so forth, was also highlighted in the current study with the identification of the need 
for exercise guidelines for cancer survivors; furthermore this idea was one rated as one of the ten most important ideas.

Increasing efforts to educate patients is a logical direction, however previous studies have shown that while patients desire information about exercise, they often do not think to broach this subject themselves (Peeters et al., 2009). This previous finding, along with other findings about oncologists' ability to elicit positive PA changes in their patients (e.g. Jones et al., 2004) and patients' preference that exercise information comes from their oncologists (e.g. Jones \& Courneya, 2002b) support the belief that oncologists may need to serve as the first line of PA promotion. This belief was echoed in the current study as the idea "Oncologists need to promote exercise” was rated higher than average on both importance and feasibility. Previous studies have hypothesized that perhaps oncologists are not promoting PA widely because they are not comfortable with the subject manner (e.g. Daley et al., 2008; Jones et al., 2005). If this hypothesis is true, then the identification by the current study's participants that oncologists need further education on the topic is a credible finding that this area needs further attention. Though, as findings of this study also indicated, there is a need for more research outcomes and further information on the dose-response relationship between activity and various outcomes. One finding that has been very consistent in the literature that may need to be conveyed to oncologists is that exercise is safe and feasible with this population (e.g. Schmitz et al., 2010).

The call for the inclusion of training on exercise and exercise promotion for health professionals is a need that has been identified by other groups and organizations whose goal is to increase the promotion of PA. For example, the National Physical Activity Plan lists this need as one of the key strategies for the health care sector to achieve the Plan's goal of increasing the PA rates of the U.S.’s population (National Physical Activity plan, 2010). Furthermore, 
physician's comfort level with providing exercise counseling is a strong predictor of whether or not they counsel their patients on increasing activity levels (Rodgers et al., 2002). The addition of training on exercise, health behavior change, and effective counseling techniques to raise PA levels could prove to be a valuable inclusion to the current physician training curriculum. Training current oncologists through conferences or other means could also prove to be a useful direction.

In addition to the ideas within the "Education" cluster, five other items stood out as being “actionable”. These items (listed in descending order by importance) were:

- “Include clinically relevant outcomes in research studies of exercise and cancer.” \#37

- “Adopt exercise into existing treatment guidelines (i.e. Clinical Pathways, NCCN).” \#14

- “Report research data from successful exercise oncology programs.” \#27

- “Define specific exercise guidelines for cancer survivors.” \#30

- "Get support from oncology organizations on the value of incorporating exercise to improve treatment outcomes.” \#16

Taken together these statements and the importance placed on them by the participants of this study point towards a gap in the research to practice continuum. Again, this gap is not unique to the subject matter at hand. In Graham et al.’s similar study investigating the promotion of PA by general practitioners (2008), which also utilized concept mapping, the call for a change in the current organizational culture was at the center of their proposed action plan which was developed from their concept mapping results. A similar culture change may need to happen within the realms of both cancer care and research being done with cancer survivors and PA to close the research to practice gap. The two populations of experts for this study all too often 
work in seclusion of each other. Further collaboration between the two groups is needed. Research should inform practice, but conversely practice should inform research so that researchers are doing the work that the physicians value. The fact that having oncology organizations support the use of exercise was seen as an important and feasible idea may indicate that this idea is a starting point for bringing these two groups together. Leaders in existing oncology organizations can reach out to researchers in hopes of further disseminating their findings and knowledge. Alike, leaders within the research community and the organizations that often support these individuals (e.g. the American College of Sports Medicine and funding organizations such as the National Institute of Health) can reach out to practicing oncologists to ensure that oncologists are playing a role in the direction of the research being done. Culture change, especially in environments such as medical practice and established research communities, is a difficult task but not an impossible one. The identification of two statements from the "Changes within the Current Oncology Environment” cluster as both important and feasible is a promising finding in that perhaps the oncology community may be open to change.

Lastly, the need for specific exercise guidelines for cancer survivors has long existed. There have been efforts to establish exercise guidelines for this population, the 2010 work of Dr. Schmitz and colleagues found, that based on the current research, physical activity (PA) for cancer survivors is both beneficial and safe. Furthermore, the recommendation for cancer survivors to follow the 2008 National PA guidelines was put forth. These findings and recommendations from this roundtable of experts perhaps need to be endorsed by Oncology organizations, and more widely distributed to oncologists in practice and in training. 


\section{Discrepancy Data between Oncologists and Researchers}

The participation of the two expert groups allows for comparisons to be made on how the two groups view the issue at hand. Overall, there was high agreement between the two groups in terms of their views of both the importance and feasibility of the items as it highlighted by the correlation scores. This finding adds value to the identification of actionable items by participants as both groups viewed the ideas similarly. Though agreement between the two groups was high, there were differences on certain items. For instance, the oncologists ranked six items as more important than average that the researchers ranked lower than average. Two of these items which possessed the greatest discrepancy were statements six and seven: "Promote home-based exercise programs," and "Provide cost-free exercise programs.” Both of these statements reside in cluster 5 "Programmatic" and the oncologists' ranking of these as important may signal their collective belief that access to exercise programming is a hindrance to further PA promotion. It also may point to a belief that PA needs to be done outside of the health care setting. Previous research investigating patients’ preference for exercise has highlighted that many patients do prefer at home exercises (e.g. Jones \& Courneya, 2002a; Maddocks, Armstrong, \& Wilcock, 2011; Rogers et al., 2008) though this finding is not universal and is often moderated by variables such as age (e.g. Jones \& Courneya, 2002a). The ranking of these ideas could be seen as a sign that perhaps the oncologists who participated believed that they did not have time to deal with patients’ activity levels or concerns, however, statement 18 “Oncologists need to promote exercise" was ranked as the $11^{\text {th }}$ most important idea by this group which gives credence to the notion that oncologists were being pragmatic with their belief that access is an important variable to consider. 
There were also six statements that the researchers rated as more important than average whereas the oncologists ranked them with a below average importance ranking. Three of these six statements came from the second cluster, "Inclusion of Exercise Professionals:”

- Train exercise and cancer specialists in community-based fitness centers. \#21

- Develop connections between healthcare providers and community-based exercise professionals. \#2

- Include exercise specialists on the cancer care team. \#35

The oncologists' contrasting view of these statements and the cluster in general may indicate a reluctant attitude towards the inclusion of professionals outside of the current cancer care team. If this attitude does exist, movement towards other ideas (i.e. Get support from oncology organizations on the value of incorporating exercise to improve treatment outcomes) may help combat this potential protective stance of the oncologists that may be inhibiting the inclusion of exercise specialists within cancer centers' walls. Though reluctance may exist to bring in other professionals, positive efforts have been made within other realms such as nutrition. The inclusion of a Registered Dietician is common among cancer care teams, which gives hope to the possible addition of exercise specialists. However, as has been seen in other areas of medicine (e.g. Cardiac Care: Certo, 1985) it may take time until this practice is widely accepted. As was suggested by the importance placed on the research cluster, the continuation of high-level research to confirm findings and investigate other areas of concerns (e.g. clinical outcomes, dose-response relationships between exercise and outcomes) is needed to provide further evidence that exercise is a powerful intervention with this population.

There was less disagreement between the two groups in terms of feasibility. However, one statement and its corresponding rating discrepancy between the two groups warrants 
discussion: “Oncologists need to promote exercise”. Oncologists viewed this idea as more feasible than the average idea while researchers viewed it as less feasible than average. While the higher than average feasibility score that the oncologists assigned to this statement may very well be a result of a bias of the sample of oncologists, this view coupled with the high importance and feasibility of many of the educations statements (specifically "Educate oncologists about the benefits of exercise”) gives hope to the idea that with some education, oncologists may be willing to include discussions about the importance of PA into their patient interactions. The potential pessimism of the research community about oncologists’ willingness to serve as catalysts for PA promotion seems to run counter to the belief of oncologists and more work educating oncologists about the benefits of exercise and how to properly promote PA could be a worthwhile avenue to explore. Oncologists such as the physicians who made up the sample for the current study may be a valuable population to enlist to encourage other practicing oncologists to promote PA. Many of these participants may have participated in the project (especially the structuring phase) because they value exercise and PA (the high amounts of PA reported by this group is another piece evidence that this group values PA) and could make these individuals ideal candidates to serve as advocates. Research and findings that come from outside this community are and will continue to be valuable, however, encouragement from other oncologists may help to increase the number of physicians who are discussing and promoting PA.

\section{Limitations}

There are a number of limitations to the current study that need to be discussed. The recruitment troubles of the brainstorming phase and the subsequent altering of the original recruitment plan for the sorting and rating is the primary limitation that affected the characteristics of the sample. Only 12 oncologists participated in the brainstorming step as 
compared with 28 researchers. The brainstorming was done anonymously, though it would be logical to believe that the researchers produced the majority of the 75 statements. Ideally, since two expert groups were being used there could have been more equal participation in the brainstorming phase. Moving forward, similar studies should better identify potential participants at the onset.

Due to the low number of oncologists who participated in the first step, the inclusion criterion of being a practicing oncologist within Appalachia was dropped. In terms of recruitment this was a necessary and fruitful step as over three times as many oncologists participated in the second phase of the study. However, many of these oncologists did not provide any ideas during the brainstorming phase. Participants who participate in both steps may possess more “ownership” over the project and as a result may approach the sorting task with more thought. Also, since participants self-selected to participate there is an increased likelihood for a selection bias. The oncologists who participated versus those who did not probably possessed different views on exercise, most likely viewing exercise in a more positive manner than their counterparts. Demographic data regarding the physical activity rates of both the researchers and oncologists supports this idea. The sample was very physical active as both groups reported participating in greater than five days of activity; this finding was found during both brainstorming and structuring. This reported amount of PA is significantly higher than that of the general population (Pleis, Ward \& Lucas, 2009).

Another potential limitation is the time that elapsed between the two participatory steps. Recruitment for brainstorming started in late August, 2011 and lasted until mid-October of that fall. Recruitment for the next phase then began in November 2011 and lasted until February, 2012. Ideally, concept mapping projects happen in a more timely fashion. This speed helps to 
keep participants engaged and helps to increase participants' familiarity with the data. Both participatory phases were open for extended periods of time to allow for continued participation. However, this gap may have prevented some participants from participating in both phases. Recruiting individuals for a participation heavy project such as this without any prior contact can be very difficult and the length of time that passed between some participants' participation in the first step and subsequent re-recruitment for the second phase may explain while only a few oncologists from the first step participated in the second and many researchers only participated in brainstorming even though they were contacted for the sorting and rating as well. Also, due to time constraints, there was no involvement of participants at the conclusion of either participatory phase. Ideally, experts are used from the stakeholder groups to both confirm that the list of ideas produced from brainstorming is robust and that the final cluster solution represents the data accurately.

Another recruitment issue is the high number of oncologists from Northern California who made up the sample of oncologists for sorting and rating $(n=16)$. This artifact is a result of the researcher having the most access to this group of oncologists (an email directory of Northern California Oncologists). This high representation may have potentially skewed the data as there may have been similarities among these oncologists that influenced the way they conceptualized the data. However, the means by which these oncologists were recruited provides an example of how future studies can recruit from this population. Oncologists and other physicians are difficult individuals to recruit for participation in research and locating existing directories or lists of these individuals can prove to be critical. Contacting and gaining permission to contact individuals through these organizations may be a needed first step for future research with this population. 


\section{Future Directions}

The "Education" cluster as a whole was found by participants to be both important and feasible. As such educational pursuits may represent the ideal first step towards the inclusion of exercise within cancer care. Specifically patients were identified as perhaps the critical group to educate. Currently means are being taken to educate this group about the benefits of exercise. It is unreasonable to believe that this group can be educated much further through organizations, pamphlets, and websites. The main source of cancer survivors’ knowledge about their disease, treatments, and protective behaviors comes from their cancer care team. As such educating oncologists and other members of the cancer care team about not only the benefits of exercise but also how to best promote PA to their patients is potentially a powerful objective to pursue.

Educational interventions aimed at increasing oncologists’ and other health professionals' knowledge of exercise with this population and information on how to best deliver this information to their patients to elicit positive changes are needed. These interventions need to be tested and shared. Ideally, interventions can be developed that can be implemented quickly and efficiently. Disseminating information through oncology organizations may represent the most effect method of reaching oncologists, at the state or national level. While education was highlighted in this study as important and feasible, education alone will not reach the goal of having exercise become part of standard cancer care.

The other clusters identified all represent areas that need to be considered. Within many of these areas participants of this study provided important and feasible ideas such as conducting larger and better designed studies. This call, which has existed for years within the literature base, has been worked towards as every year researchers are employing more rigorous methodological procedures with larger samples. Researchers must continue this trend and 
continue to identify relevant outcome variables. However, attention also needs to be paid to the interventions themselves with the goal of developing pragmatic interventions that fit within patients' lives and within the current treatment environment. Changes are needed to the oncology environment as signified by the formation of this area as a cluster and the formation of an advocate group of practicing physicians may help to start these changes, but as is evident by the slow path throughout decades that cardiac rehabilitation took to become a standard part of care, these changes may take time (Certo, 1985). It takes more than education, and research, and motivated physicians to change ways of practice and thinking. Bringing about this change will take a multifaceted approach and this study highlighted a number of these dimensions that need to be addressed for exercise to become part of standard cancer care. The placement of the “Changes within the Current Oncology Environment” at the center of the map reinforces this notion. The centrality of this cluster and the ideas that it contains is a potential outcome of the belief that the other clusters and ideas are related to changing the culture. If exercise were to become part of standard cancer care it would be because the current culture has changed. Though this may take time, as this study has highlighted, if advancement can be achieved in various fields this change can be realized. 


\section{References}

Adamsen, L., Quist, M., Andersen, C., Moller, T., Herrstedt, J., Kronborg., . . Rorth, M. (2009). Effect of a multimodal high intensity exercise intervention in cancer patients undergoing chemotherapy: Randomized controlled trial. British Medical Journal, 339, 895-899.

American Cancer Society, (2011). Exercise. Retrieved from:

http://www.cancer.org/Treatment/TreatmentsandSideEffects/PhysicalSideEffects/Dealing withSymptomsatHome/caring-for-the-patient-with-cancer-at-home-exercise.

Anderson, L.A., Gwaltney, M.K., Sundra, D.L., Brownson, R.C., Kane, M, Cross, A.W., .. . White, C.R. (2006). Using concept mapping to develop a logic model for the prevention research centers program. Preventing Chronic Disease, 3, 1-09.

Association of Community Cancer Centers (2011). ACCC member cancer programs. Retrieved from: http://www.accc-cancer.org/membership_directory/default.asp

Battersby, M., Von Korff, M., Schaefer, J., Davis, C., Ludman, E., Greene, S.M., ... Wagner, E.H. (2010). Twelve evidence-based principles for implementing self-management support in primary care. The Joint Commission Journal on Quality and Patient Safety, 36, 561-570.

Boffetta, P., Hecht, S., Gray, N., Gupta, P., \& Straif, K. (2008). Smokeless tobacco and cancer. The Lancet Oncology, 9, 667-675.

Burke, J.G., O’Campo, P., Peak, G.L., Gielen, A.C., McDonnell, K.A., \& Trochim, W.M.K. (2005). An introduction to concept mapping as a participatory public health research method. Qualitative Health Research, 15, 1392-1410.

Center for Disease Control. (2010). Cancer survivorship. Retrieved from http://www.cdc.gov/cancer/survivorship/pdf/survivorship_fs_2010.pdf 
Certo, C.M. (1985). History of cardiac rehabilitation. Physical Therapy, 65, 1793-1795.

Concept Systems Global [Computer software]. Ithaca, NY: Concept Systems Incorporated.

Courneya, K.S. \& Friedenreich, C.M. (1997a). Relationship between exercise during treatment and current quality of life among survivors of breast cancer. Psychosocial Oncology, 15, 35-37.

Courneya, K.S. \& Friedenreich, C.M. (1997b). Relationship between exercise pattern across the cancer experience and current quality of life in colorectal cancer survivors. Alternative Complementary Medicine, 3, 215-226.

Daley, A.J., Crank, H., Saxton, J.M., Mutrie, N., Coleman, R. \& Roalfe, A. (2007). Randomized trial of exercise therapy in women treated for breast cancer. Journal of Clinical Oncology, 13, 1713-1721.

Daley, A.J., Bowden, S.J., Rea, D.W., Billingham, L. \& Carmicheal, A.R. (2008). What advice are oncologists and surgeons in the United Kingdom giving to breast cancer patients about physical activity? International Journal of Behavioral Nutrition and Physical Activity, 5, 46. Online publication.

Demark-Wahnefried, W., Aziz, N.M., Rowland, J.H. \& Pinto, B.M. (2005). Riding the crest of the teachable moment: Promoting long-term health after the diagnosis of cancer. Journal of Clinical Oncology, 23, 5814-5830.

Graham, A.L., Kerner, J.F., Quinlan, K.M., Vinson, C., \& Best, A. (2008). Translating cancer control research into primary care practice: A conceptual framework. American Journal of Lifestyle Medicine, 2, 241-249. 
Holick, C.N., Newcomb, P.A., Trentham-Dietz, A, Titus-Ernstoff, L., Bersch, A.J., Stampfer, M.J., . . . Willett, W.C. (2008). Physical activity and survival after diagnosis of invasive breast cancer. Cancer Epidemiological Biomarkers Prevention, 17, 379-386.

Holmes, M.D., Chen, W.Y., Feskanich, D., Kroenke, C.H., \& Colditz, G.A. (2005). Physical activity and survival after breast cancer diagnosis. JAMA, 293, 2479-2486.

Jemel, A., Siegel, R., Xu, J. \& Ward, E. (2010). Cancer statistics, 2010. CA: A Cancer Journal for Clinicians, 60, 277-300.

Jones, L.W. \& Courneya, K.S. (2002a). Exercise counseling and programming preferences of cancer survivors. Cancer Practice, 10, 208-215.

Jones L.W. \& Courneya, K.S. (2002b). Exercise discussions during cancer treatment consultations. Cancer Practice, 10, 66-74.

Jones, L.W., Courneya, K.S., Fairy, A.S. \& Mackey, J.R. (2004). Effects of an oncologist’s recommendation to exercise on self-reported exercise behavior in newly diagnosed breast cancer survivors: A single-blind, randomized controlled trial. Annals of Behavioral Medicine, 28, 105-113.

Jones, L.W., Courneya, K.S., Peddle, C. \& Mackey, J.R. (2005). Oncologists’ opinions towards recommending exercise to patients with cancer: a Canadian national survey. Supportive Care in Cancer, 13, 929-937.

Kane, M. \& Trochim, W.M.K. (2007). Concept mapping for planning and evaluation. Thousand Oaks, CA: Sage.

Lynch, B.M. (2010). Sedentary behavior and cancer: A systematic review of the literature and proposed biologic mechanism. Cancer Epidemiology, Biomarkers, and Prevention, 19, 2691-2709. 
Maddocks, M., Armstrong, S. \& Wilcock, A. (2011). Exercise as a supportive therapy in incurable cancer: Exploring patient preferences. Psychooncology, 2, 173-180.

Meadows, A.T., Varricchio, C., Crosson, K., Harlan, L., McCormick, P., Nealon, E., .. . Ungerleider, R. (1998). Research issues in cancer survivorship: Report of a workshop sponsored by the office of cancer survivorship, national cancer institute. Cancer Epidemiology, Biomarkers \& Prevention, 7, 1145-1151.

Milne, H.M., Wallman, K.E., Gordon, S. \& Courneya, K.S. (2008). Effects of a combined aerobic and resistance exercise program in breast cancer survivors: A randomized controlled trial. Breast Cancer Research and Treatment, 108, 279-288.

Mutrie, N., Campbell, A.M., Whyte, F, McConnachie, A, Emslie, C, Lee, L., . . Ritchie, D. (2007). Benefits of supervised group exercise programme for women being treated for early stage breast cancer: Pragmatic randomized controlled trial. British Medical Journal, 334, 517-524.

National Physical Activity Plan. (2010). National physical activity plan. Retrieved from: http://www.physicalactivityplan.org/NationalPhysicalActivityPlan.pdf

National Cancer Institute. (2012). All community clinical oncology programs (CCOPs) grantees. Retrieved from http://prevention.cancer.gov/research-networks/ccop/index.htm

Peeters, C., Stewart, A., Segal, R., Wouterloot, E., Scott, C.G., \& Aubry, T. (2009). Evaluation of a caner exercise program: Patient and physician beliefs. Psycho-Oncology, 18, 898902.

Pleis, J.R., Ward, B.W. \& Lucas, J.W. (2010). Summary health statistics for U.S. adults: National health interview survey, 2009. Vital Health Statistics, 249, 10, 1-207. 
Rogers, L.Q., Bailey, J.E., Gutin, B., Johnson, K.C., Levine, M.A., Milan, F., . . Sherman, S.E. (2002). Teaching resident physicians to provide exercise counseling: A needs assessment. Academic Medicine, 77, 841-844.

Rogers, L.Q., Courneya, K.S., Verhulst, S., Markwell, S., Lanzotti, V. \& Shah, P. (2008). Exercise barrier and task self-efficacy in breast cancer patients during treatment. Supportive Cancer Care, 14, 84-90.

Schmitz, K.H, Courneya, K.S., Matthews, C., Demark-Wahnefried, W., Galvao, D.A., Pinto, B.M., . . Schwartz, A.L. (2010). American college of sports medicine roundtable on exercise guidelines for cancer survivors. Medicine and Science in Sports and Exercise, 42, 1409-1426.

Segal, R.J., Reid, R.D., Courneya, K.S., Sigal, R.J., Kenny, G.P., Prud’Homme, D.G., . . . Slovinec D’Angelo, M.E. (2009). Randomized controlled trial of resistance or aerobic exercise in men receiving radiation therapy for prostate cancer. Journal of Clinical Oncology, 27,344- 351.

Speck, R.M., Courneya, K.S., Masse, L.C., Duval, S. \& Schmitz, K.H. (2010). An update of controlled physical activity trials in cancer survivors: a systematic review and metaanalysis. Journal of Cancer Survivorship, 4, 87-100.

Sternfeld, B., Weltzien, E., Quesenberry, C.P., Castiollo, A.L., Kwan, M., Slattery, M.L. \& Caan, B.J. (2009). Physical activity and risk of recurrence and mortality in breast cancer survivors: Findings from the LACE study. Cancer Epidemiological Biomarkers Prevention, 18, 87-95. 
Stillman, F. Hoang, M., Linton, B., Ritthiphakdee, B. \& Trochim, W. (2008). Mapping tobacco industry strategies in south east Asia for action planning and surveillance. Tobacco Control, 17, 1-7. Online publication.

Tong, E.K., Strouse, R., Hall, J., Kovak, M. \& Schroeder, S.A. (2010). National survey of U.S. health professionals’ smoking prevalence, cessation practices, and beliefs. Nicotine and Tobacco Research, Advance online publication.

Trochim, W.M.K. (1989). An introduction to concept mapping for planning and evaluation. Evaluation and Program Planning, 12, 1-16.

Trochim, W.M.K. (1993). Reliability of concept mapping. Paper presented at the Annual Conference of the American Evaluation Association, Dallas, Texas, retrieved from: http://www.socialresearchmethods.net/research/Reliable/reliable.htm.

Trochim, W.M.K., Milstein, B., Wood, B.J., Jackson, S., \& Pressler, V. (2004). Setting objectives for community and systems change: An application of concept mapping for planning a statewide health improvement initiative. Health Promotion Practice, 5, 8-19. 
Table 1

Brainstorming Demographics

\begin{tabular}{|c|c|c|c|}
\hline Oncologists $n=12$ & & Researchers $n=26$ & \\
\hline Experience (years) & $17.17(12.92)$ & Experience (years) & $8.42(4.87)$ \\
\hline $\begin{array}{l}\text { Moderate PA (Days } \\
\text { per week of at least } \\
30 \text { min) }\end{array}$ & $3.60(1.90)$ & $\begin{array}{l}\text { Number of studies } \\
\text { regarding cancer and } \\
\text { exercise }\end{array}$ & 9.96 (11.35) \\
\hline $\begin{array}{l}\text { Vigorous PA (Days } \\
\text { per week of at least } \\
20 \text { min) }\end{array}$ & $2.27(2.05)$ & $\begin{array}{l}\text { Moderate PA (Days } \\
\text { per week of at least } \\
30 \text { min) }\end{array}$ & $4.65(2.04)$ \\
\hline Gender & & $\begin{array}{l}\text { Vigorous PA (Days } \\
\text { per week of at least } \\
20 \mathrm{~min} \text { ) }\end{array}$ & $3.29(2.01)$ \\
\hline Male & $n=9$ & Gender & \\
\hline Female & $n=2$ & Male & $n=3$ \\
\hline Setting & & Female & $n=21$ \\
\hline $\begin{array}{l}\text { Community } \\
\text { Cancer Center }\end{array}$ & $n=10$ & & \\
\hline $\begin{array}{l}\text { Academic Cancer } \\
\text { Center }\end{array}$ & $n=2$ & & \\
\hline \multicolumn{4}{|l|}{ Oncology Type } \\
\hline Surgical & $n=2$ & & \\
\hline Radiation & $n=3$ & & \\
\hline Medical & $n=7$ & & \\
\hline
\end{tabular}

Note. Unless specified, data are presented as mean (standard deviation). 
Table 2

Structuring Demographics

\begin{tabular}{lll}
\hline & Oncologists $n=38$ & Researchers $n=20$ \\
\hline $\begin{array}{l}\text { Experience (years) } \\
\text { Moderate PA (Days per }\end{array}$ & $21.11(11.54)$ & $16.50(9.26)$ \\
$\begin{array}{l}\text { week of at least 30 min) } \\
\text { Vigorous PA (Days per }\end{array}$ & $3.05(2.08)$ & $4.95(1.28)$ \\
$\begin{array}{l}\text { week of at least 20 min) } \\
\text { Cancer type }\end{array}$ & $3.15(1.46)$ \\
Breast & $n=24$ & \\
Colon & $n=2$ & $n=12$ \\
Lung & $n=5$ & $n=2$ \\
Prostate & $n=2$ & $n=0$ \\
Other & $n=5$ & $n=3$ \\
\hline
\end{tabular}

Note. Unless specified data are presented as mean (standard deviation). 
Table 3

List of Statements

\begin{tabular}{|c|c|c|}
\hline \# & Statement & $\begin{array}{l}\text { Bridging } \\
\text { Value }\end{array}$ \\
\hline 1 & Educate oncologists about the benefits of exercise. & 0.42 \\
\hline 2 & $\begin{array}{l}\text { Develop connections between healthcare providers and community-based } \\
\text { exercise professionals. }\end{array}$ & 0.33 \\
\hline 3 & Educate the cancer care team about the benefits of exercise. & 0.39 \\
\hline 4 & $\begin{array}{l}\text { Mandate referrals to exercise programs much like the current cardiac } \\
\text { rehabilitation model. }\end{array}$ & 0.43 \\
\hline 5 & Train members of the cancer care team on how to properly promote exercise. & 0.55 \\
\hline 6 & Promote home-based exercise programs. & 0.10 \\
\hline 7 & Provide cost-free exercise programs. & 0.00 \\
\hline 8 & Develop exercise centers for cancer survivors. & 0.03 \\
\hline 9 & $\begin{array}{l}\text { Confirm the various mechanisms (e.g. biological) that are involved with the } \\
\text { benefits of exercise for cancer survivors. }\end{array}$ & 0.16 \\
\hline 10 & $\begin{array}{l}\text { Develop systems-oriented intervention approaches that rely less on the } \\
\text { oncologists and more on support staff. }\end{array}$ & 0.38 \\
\hline 11 & Stay away from a medical model of rehabilitation. & 0.32 \\
\hline 12 & Make exercise billable to health insurance companies. & 0.45 \\
\hline 13 & Prioritize recovery issues. & 0.52 \\
\hline 14 & $\begin{array}{l}\text { Adopt exercise into existing treatment guidelines (i.e. Clinical Pathways, } \\
\text { NCCN). }\end{array}$ & 0.57 \\
\hline 15 & Educate patients about the benefits of exercise & 1.00 \\
\hline 16 & $\begin{array}{l}\text { Get support from oncology organizations on the value of incorporating } \\
\text { exercise to improve treatment outcomes. }\end{array}$ & 0.54 \\
\hline 17 & Review the history of exercise as part of cardiac rehabilitation. & 0.82 \\
\hline 18 & Oncologists need to promote exercise. & 0.52 \\
\hline 19 & Look at the LIVESTRONG at YMCA model of exercise. & 0.66 \\
\hline 20 & $\begin{array}{l}\text { Have cancer patients engage in supervised physical activity at the doctor's } \\
\text { office so oncologists can see that patients can exercise without getting } \\
\text { injured. }\end{array}$ & 0.99 \\
\hline 21 & Train exercise and cancer specialists in community-based fitness centers. & 0.63 \\
\hline 22 & $\begin{array}{l}\text { Staff exercise programs with trainers who have certifications to work with } \\
\text { cancer survivors. }\end{array}$ & 0.18 \\
\hline 23 & Focus on cost-effectiveness studies. & 0.82 \\
\hline 24 & Provide group opportunities for exercise. & 0.06 \\
\hline 25 & Increase funding for exercise interventions and programming. & 0.36 \\
\hline 26 & $\begin{array}{l}\text { Provide evidence on how to prescribe exercise for specific cancer-related } \\
\text { outcomes. }\end{array}$ & 0.51 \\
\hline 27 & Report research data from successful exercise oncology programs. & 0.28 \\
\hline 28 & $\begin{array}{l}\text { Provide patients with the support (i.e. facilities, education, behavioral } \\
\text { support) needed to engage in safe exercise. }\end{array}$ & 0.13 \\
\hline
\end{tabular}


Table 3 continued...

29 Develop effective activity interventions that don't require patients to go to 0.44 clinic-based facilities.

30 Define specific exercise guidelines for cancer survivors. $\quad 0.75$

31 Publicize testimonials of patients and oncologists. $\quad 0.78$

32 Develop appropriate interventions based around patients' treatment and 0.72 survivorship issues.

33 Have therapists help patients start exercise programs 0.13

34 Produce more evidence regarding the exact type, dose and timing of exercise $\quad 0.09$ needed.

35 Include exercise specialists on the cancer care team. 0.40

36 Conduct larger, better designed studies to confirm current findings. 0.09

37 Include clinically relevant outcomes in research studies of exercise and $\quad 0.10$

Note. Bridging values $(0-1)$ are used to determine if a statement is considered to be an anchor or bridging statement. Statements that are anchor statements are considered to be reflective of the statements that are close to it on the map where as bridging statements link areas of the map together. Lower values are indicative of anchor statements. 
Table 4

Statements by Cluster

\begin{tabular}{rlr}
\hline$\#$ & \multicolumn{1}{c}{ Statement } & $\begin{array}{c}\text { Bridging } \\
\text { Value }\end{array}$ \\
\hline Cluster 1 - Education & 0.39 \\
3 & Educate the cancer care team about the benefits of exercise. & 0.42 \\
1 & Educate oncologists about the benefits of exercise. & 0.52 \\
18 & Oncologists need to promote exercise. & 0.55 \\
5 & Train members of the cancer care team on how to properly promote exercise. & 0.78 \\
31 & Publicize testimonials of patients and oncologists. & 1.00 \\
15 & Educate patients about the benefits of exercise
\end{tabular}

Count: 6 SD: $0.21 \quad$ Mean: $0.61 \quad$ Med: 0.54

Cluster 2 - Inclusion of Exercise Professionals

2 Develop connections between healthcare providers and community-based exercise professionals.

35 Include exercise specialists on the cancer care team.

21 Train exercise and cancer specialists in community-based fitness centers. 0.63

20 Have cancer patients engage in supervised physical activity at the doctor's $\quad 0.99$ office so oncologists can see that patients can exercise without getting injured.

Count: 4 SD: $0.26 \quad$ Mean: $0.59 \quad$ Med: 0.51

Cluster 3 - Changes within the Current Oncology Environment

10 Develop systems-oriented intervention approaches that rely less on the oncologists and more on support staff.

13 Prioritize recovery issues.

16 Get support from oncology organizations on the value of incorporating exercise to improve treatment outcomes.

14 Adopt exercise into existing treatment guidelines (i.e. Clinical Pathways, $\quad 0.57$ NCCN).

17 Review the history of exercise as part of cardiac rehabilitation.

Count: 5 SD: $0.14 \quad$ Mean: $0.56 \quad$ Med: 0.54

Cluster 4 - Research

36 Conduct larger, better designed studies to confirm current findings. 0.09

34 Produce more evidence regarding the exact type, dose and timing of exercise $\quad 0.09$ needed.

37 Include clinically relevant outcomes in research studies of exercise and 0.10 cancer.

9 Confirm the various mechanisms (e.g. biological) that are involved with the benefits of exercise for cancer survivors.

27 Report research data from successful exercise oncology programs. 0.28

26 Provide evidence on how to prescribe exercise for specific cancer-related $\quad 0.51$ outcomes.

23 Focus on cost-effectiveness studies.

0.82

Count: 7 SD: $0.26 \quad$ Mean: $0.29 \quad$ Med: 0.16


Table 4 continued...

Cluster 5 - Needed Components of Exercise Programs

7 Provide cost-free exercise programs. $\quad 0.00$

8 Develop exercise centers for cancer survivors. 0.03

24 Provide group opportunities for exercise. $\quad 0.06$

6 Promote home-based exercise programs. $\quad 0.10$

28 Provide patients with the support (i.e. facilities, education, behavioral 0.13 support) needed to engage in safe exercise.

33 Have therapists help patients start exercise programs 0.13

22 Staff exercise programs with trainers who have certifications to work with 0.18 cancer survivors.

11 Stay away from a medical model of rehabilitation. $\quad 0.32$

25 Increase funding for exercise interventions and programming. $\quad 0.36$

4 Mandate referrals to exercise programs much like the current cardiac 0.43 rehabilitation model.

12 Make exercise billable to health insurance companies.

0.45

Count: 11 SD: $0.16 \quad$ Mean: $0.20 \quad$ Med: 0.13

Cluster 6 - Patient Focused

29 Develop effective activity interventions that don't require patients to go to 0.44 clinic-based facilities.

19 Look at the LIVESTRONG at YMCA model of exercise. 0.66

32 Develop appropriate interventions based around patients' treatment and $\quad 0.72$ survivorship issues.

30 Define specific exercise guidelines for cancer survivors. 0.75 Count: 4 SD: $0.12 \quad$ Mean: $0.64 \quad$ Med: 0.69

Note. Bridging values $(0-1)$ are used to determine if a statement is considered to be an anchor or bridging statement. Statements that are anchor statements are considered to be reflective of the statements that are close to it on the map where as bridging statements link areas of the map together. Lower values are indicative of anchor statements. 


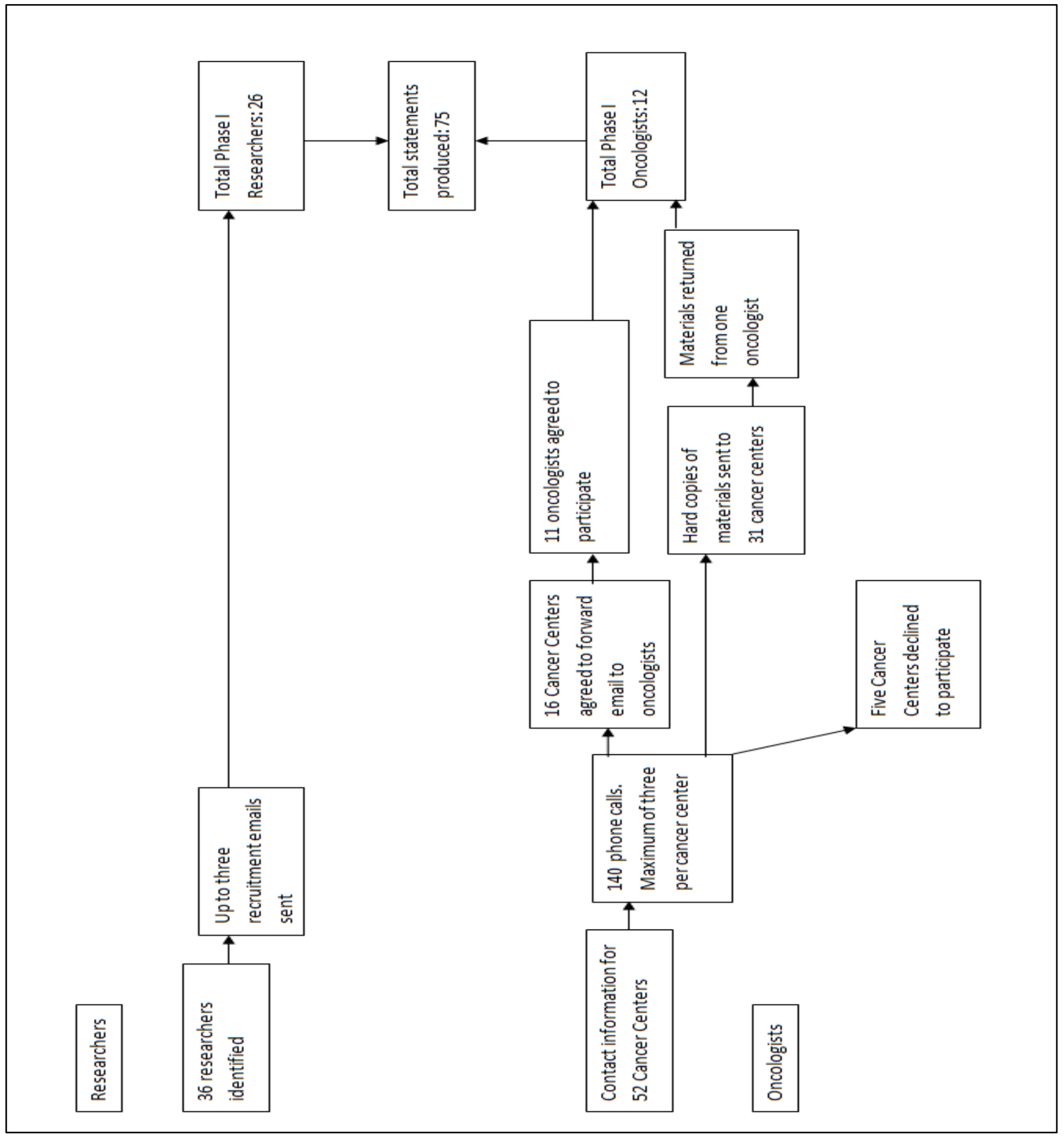

Figure 1. Recruitment flow chart for the brainstorming process. 


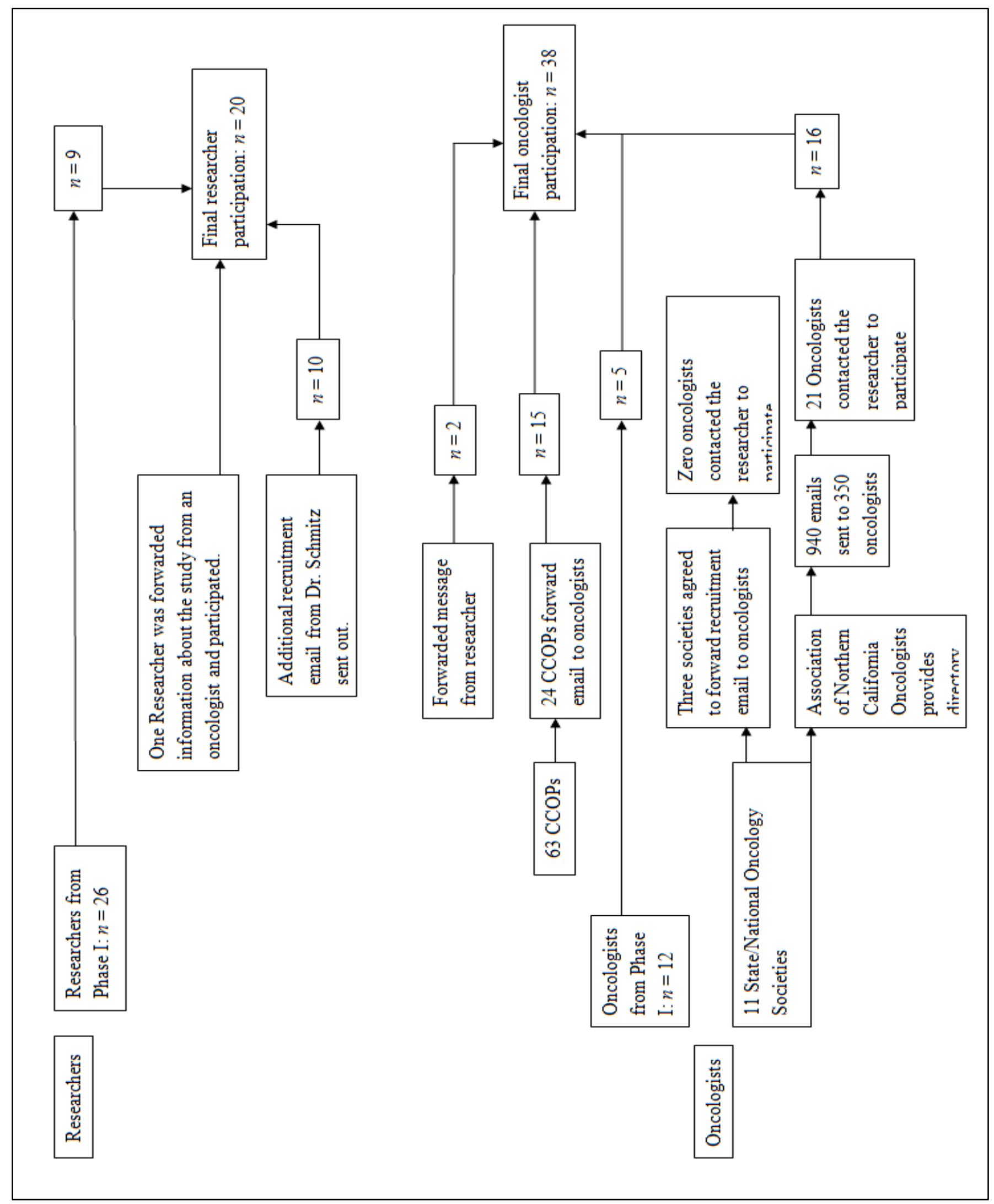

Figure 2. Recruitment flow chart for the structuring process. 


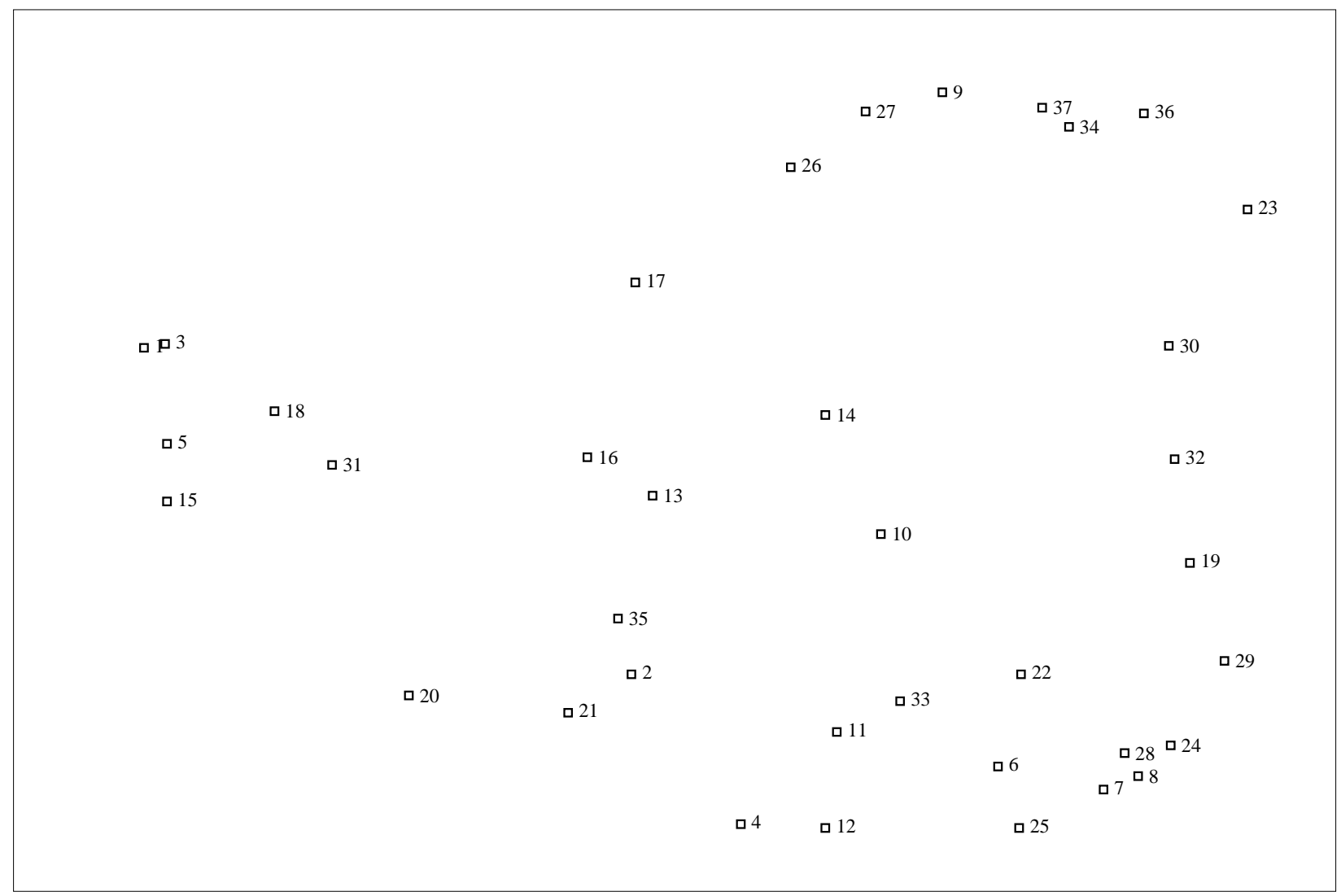

Figure 3. Point map of the statement data. Each number refers to a statement. Statements that are close geographically on the map were sorted together often (e.g. statements 1 \& 3) whereas statements further apart were sorted together less often (e.g. statements 5 and 32). 


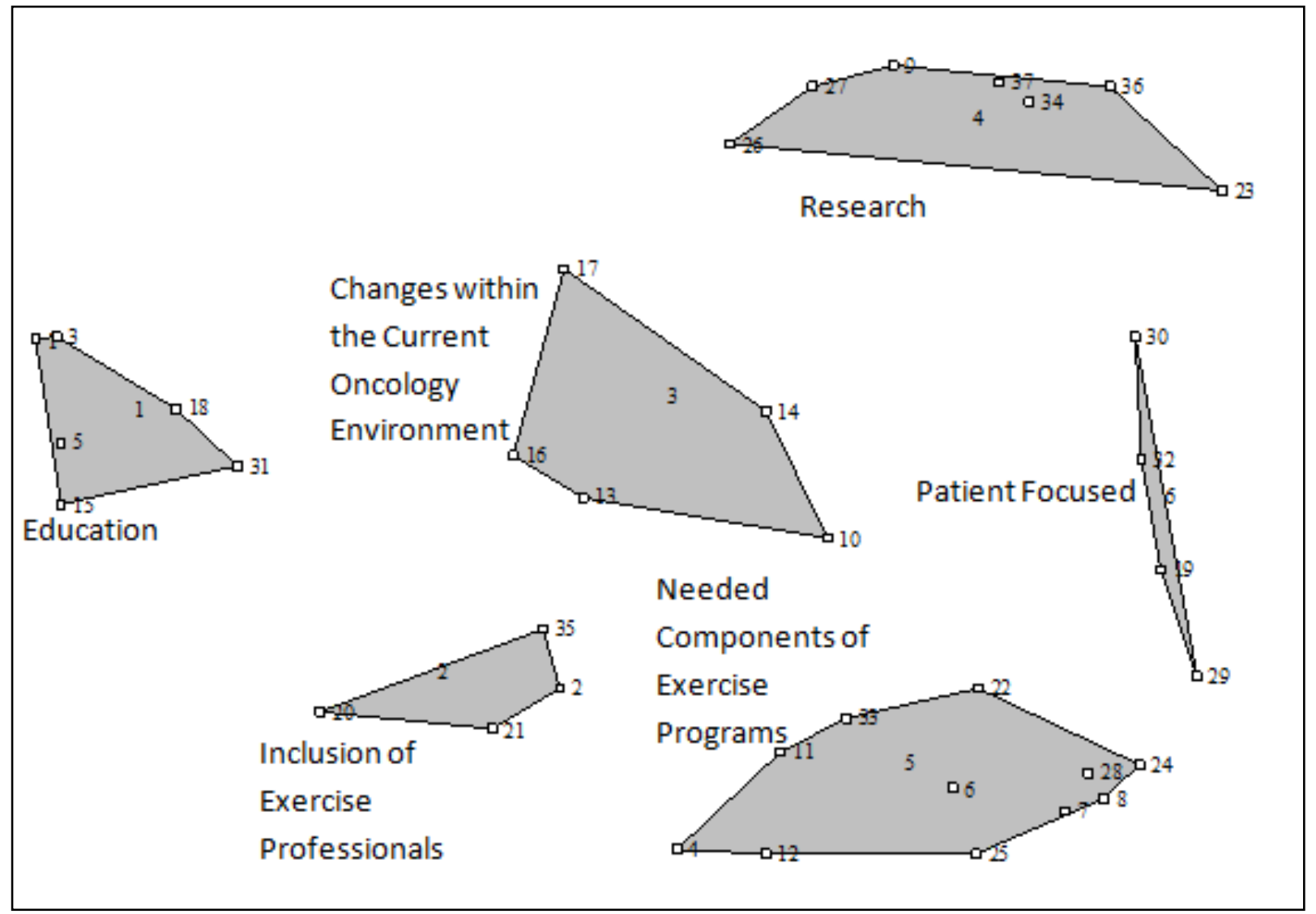

Figure 4. Six cluster solution cluster map. Clarification: cluster 3 = "Changes within the Current Oncology Environment,” cluster 5 = “Needed Components of Exercise Programs.” 


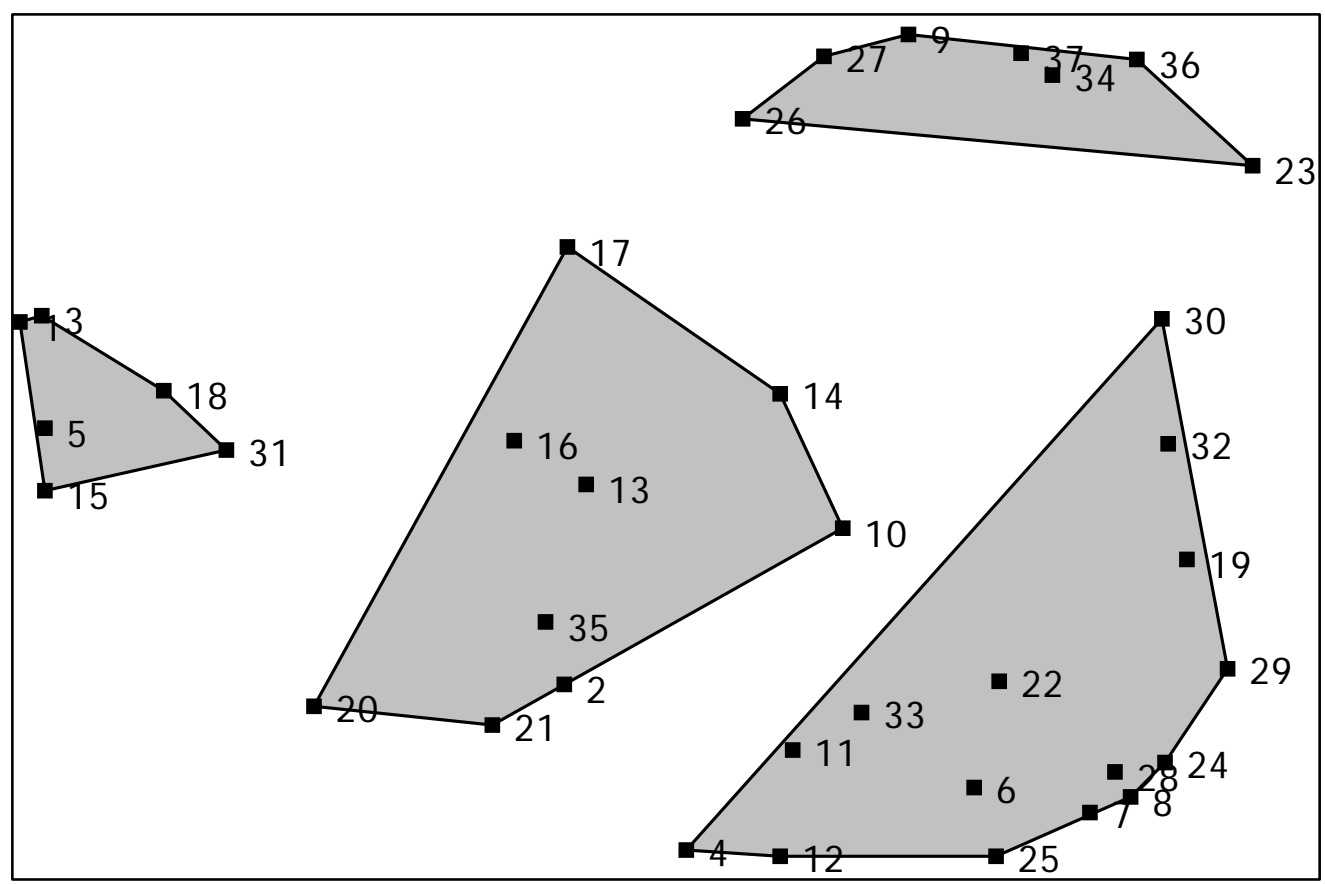

Figure 5. Four-cluster solution. 


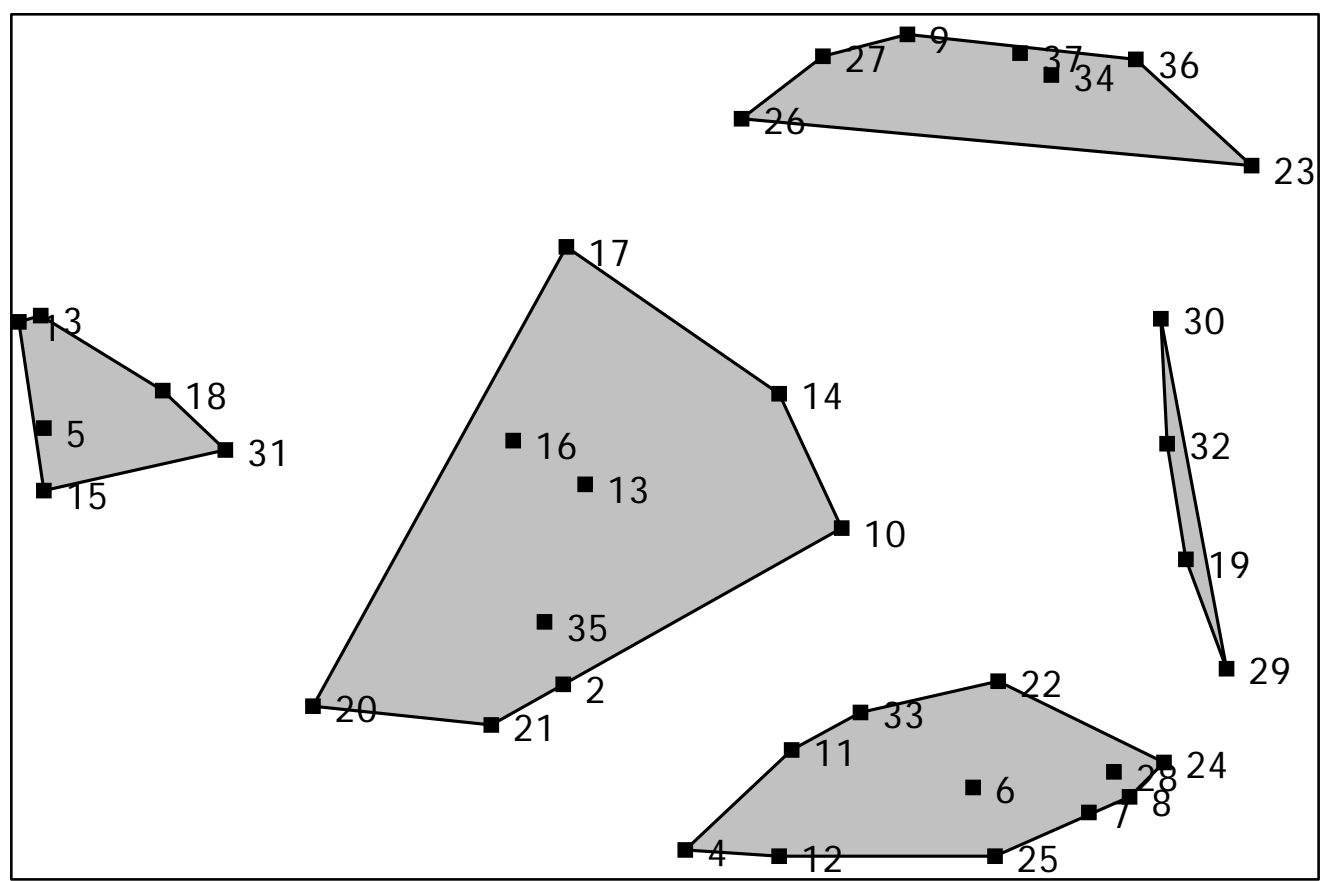

Figure 6. Five-cluster solution. 


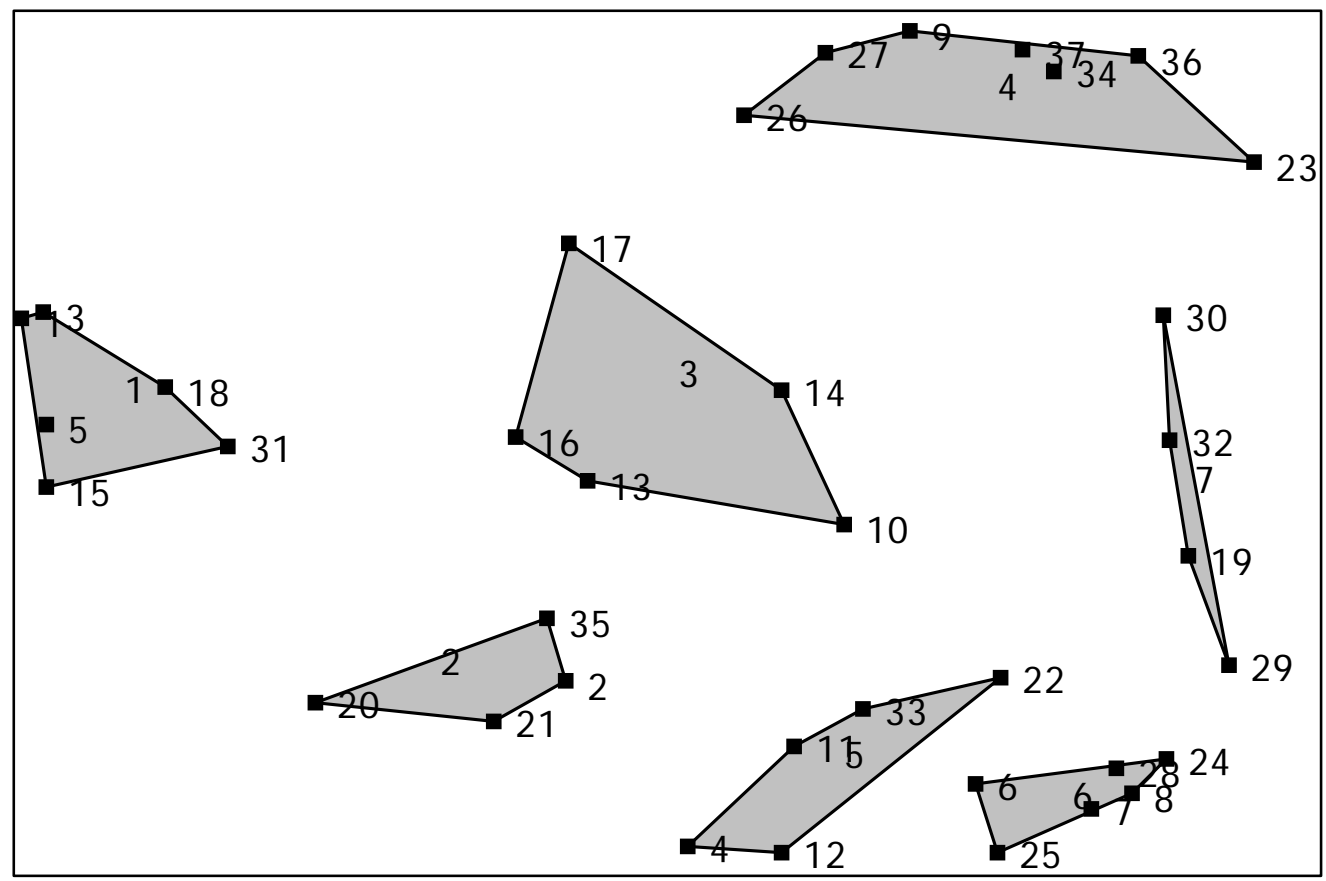

Figure 7.Seven-cluster solution. 


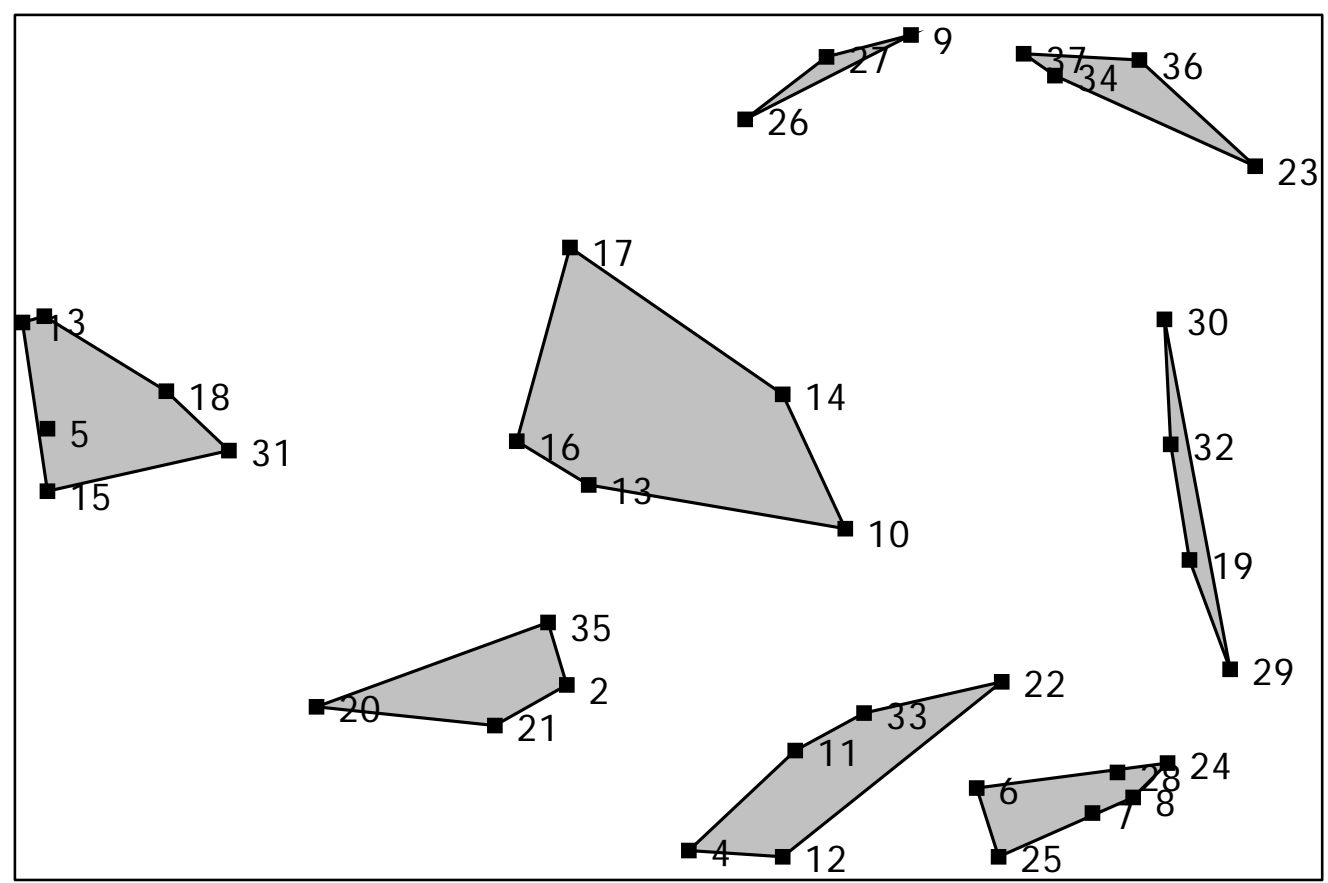

Figure 8. Eight-cluster solution. 


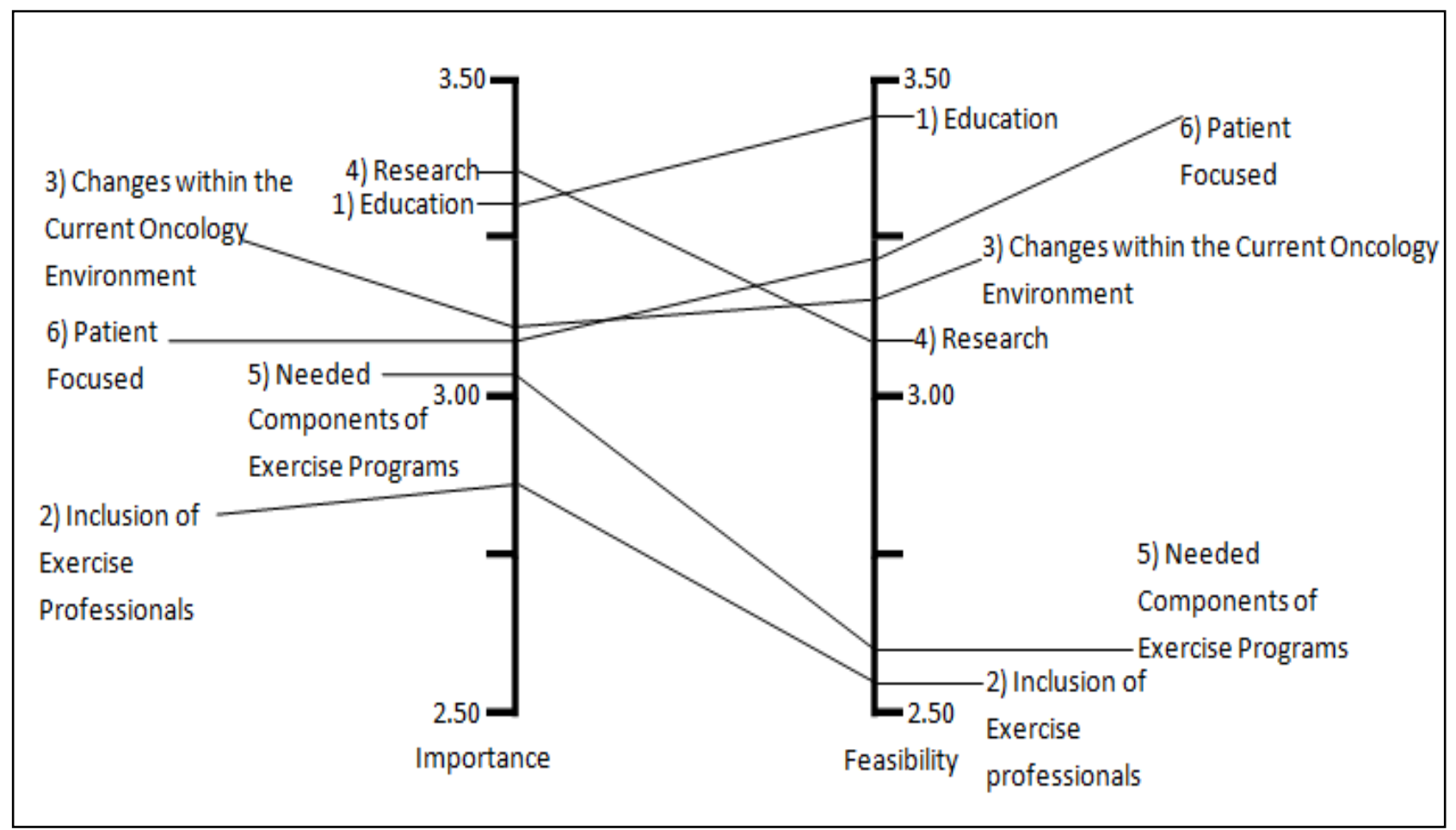

Figure 9. Pattern-match display depicting importance and feasibility ratings of the six clusters. Each clusters' position on the ladder graph is representative of its average rating on the given variable (importance or feasibility) as rated by all of the individuals who completed the rating step of the project. 


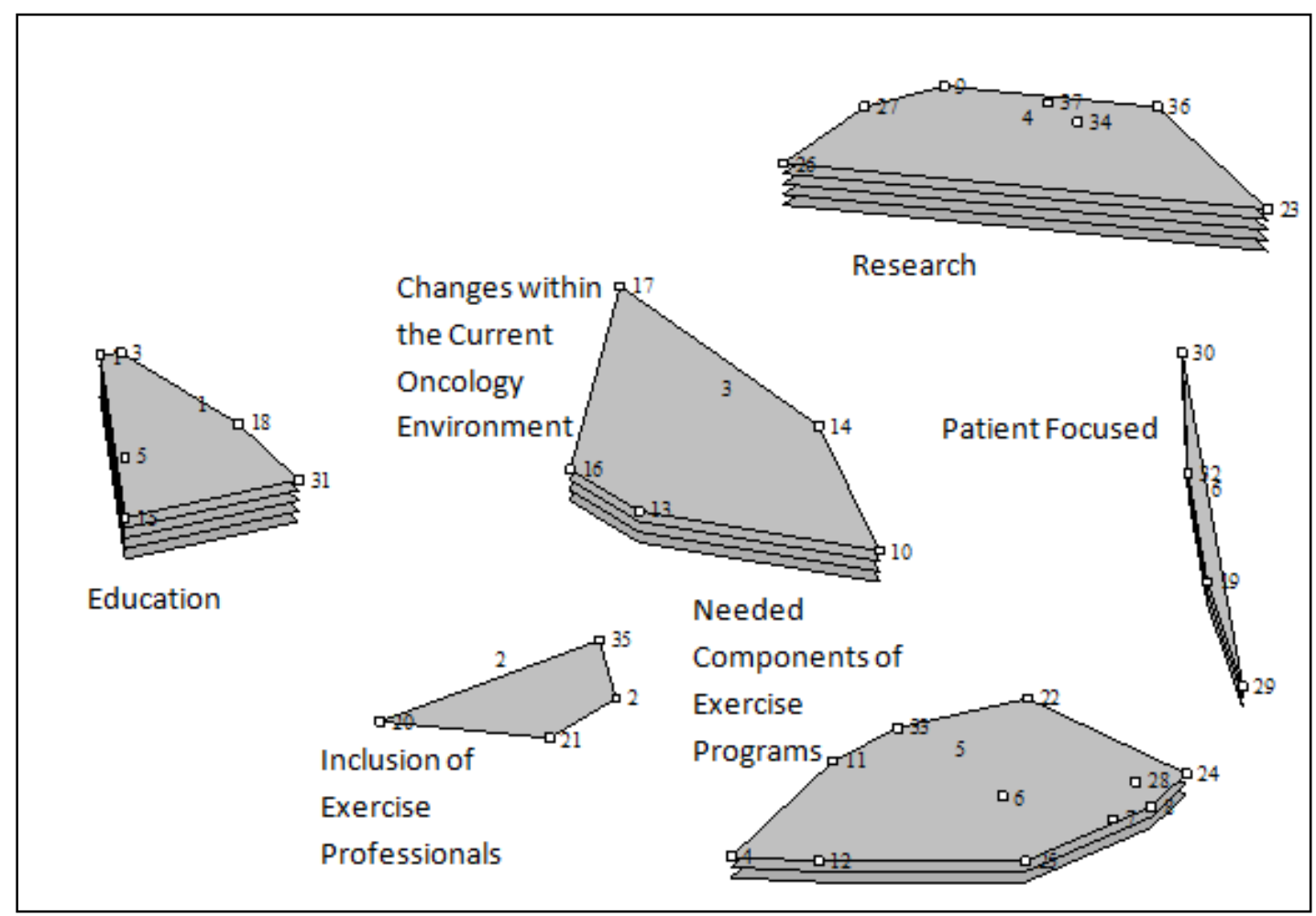

Figure 10. Cluster rating map for importance. Clusters which have larger "stacks” possess higher average ratings of importance. 


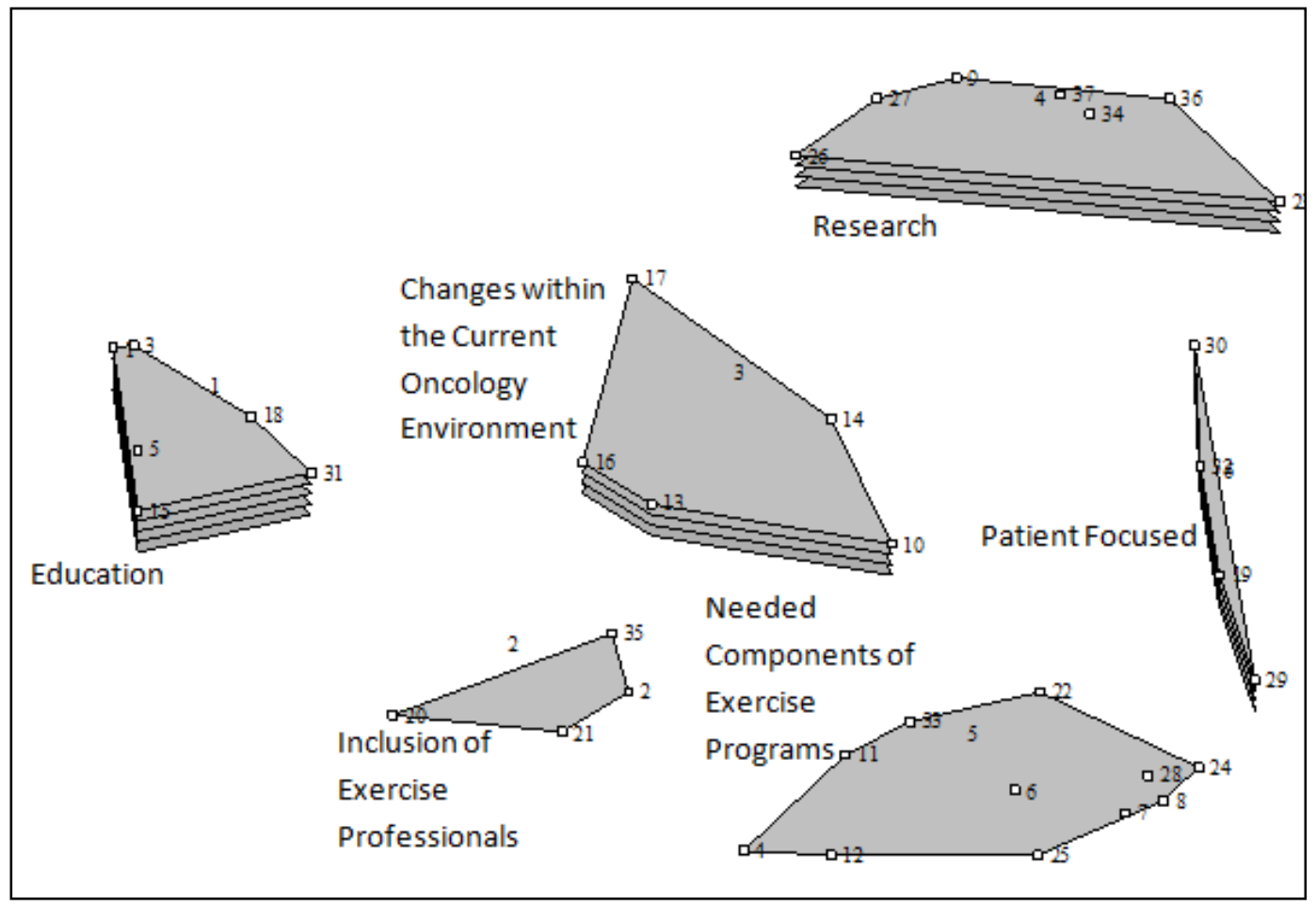

Figure 11. Cluster rating map for feasibility. Clusters which have larger "stacks" possess higher average ratings of feasibility. 


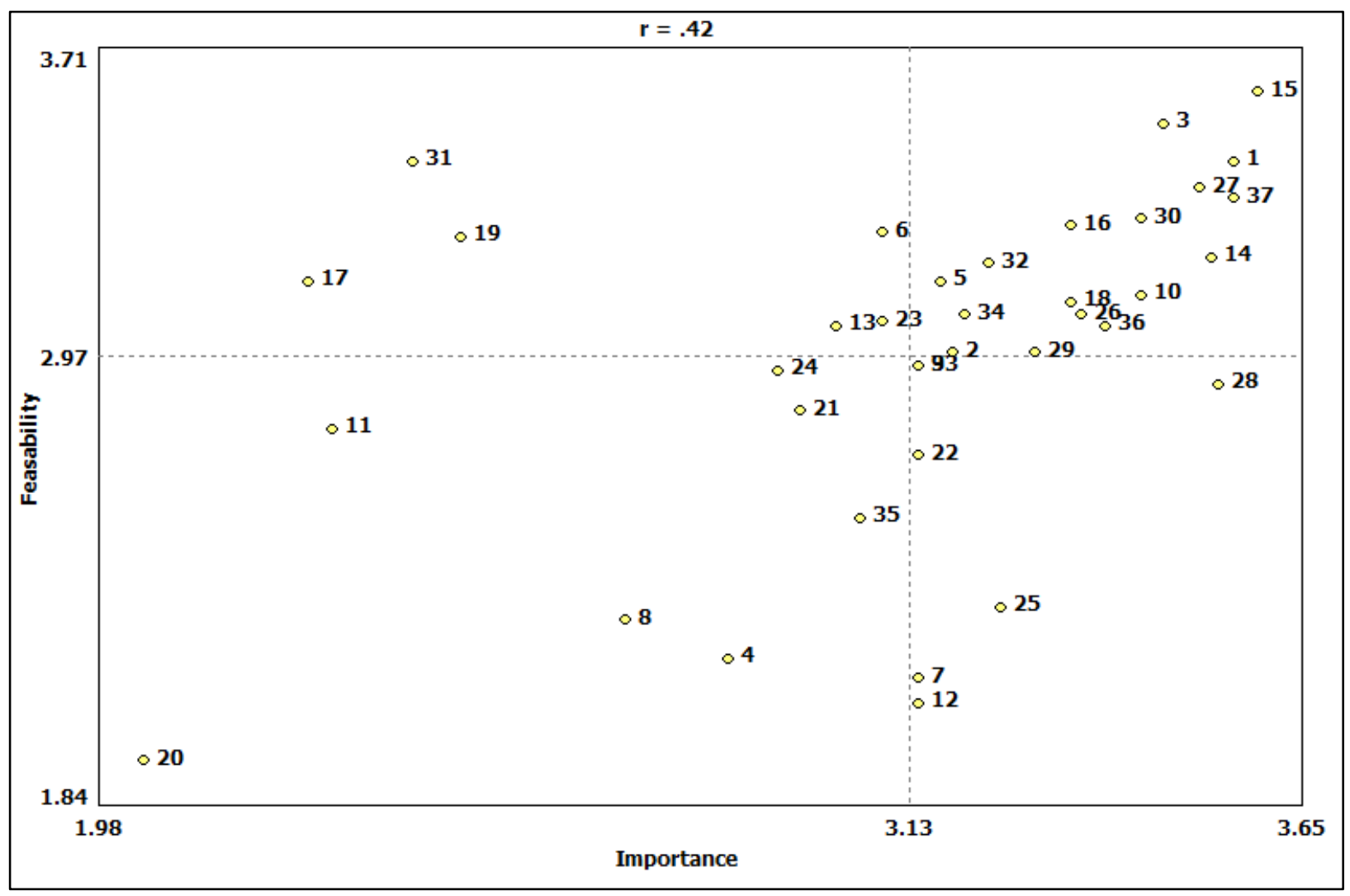

Figure 12. Go-zone display depicting the average rating of every statement on both importance (x-axis) and feasibility (y-axis). The display is broken into four quadrants based on the average rating of importance (3.13) and feasibility (2.97) based on all the participants' responses.

Statements in quadrant one (the upper right) possess higher than average ratings on both of these variables and as such are considered to be the most "actionable." 


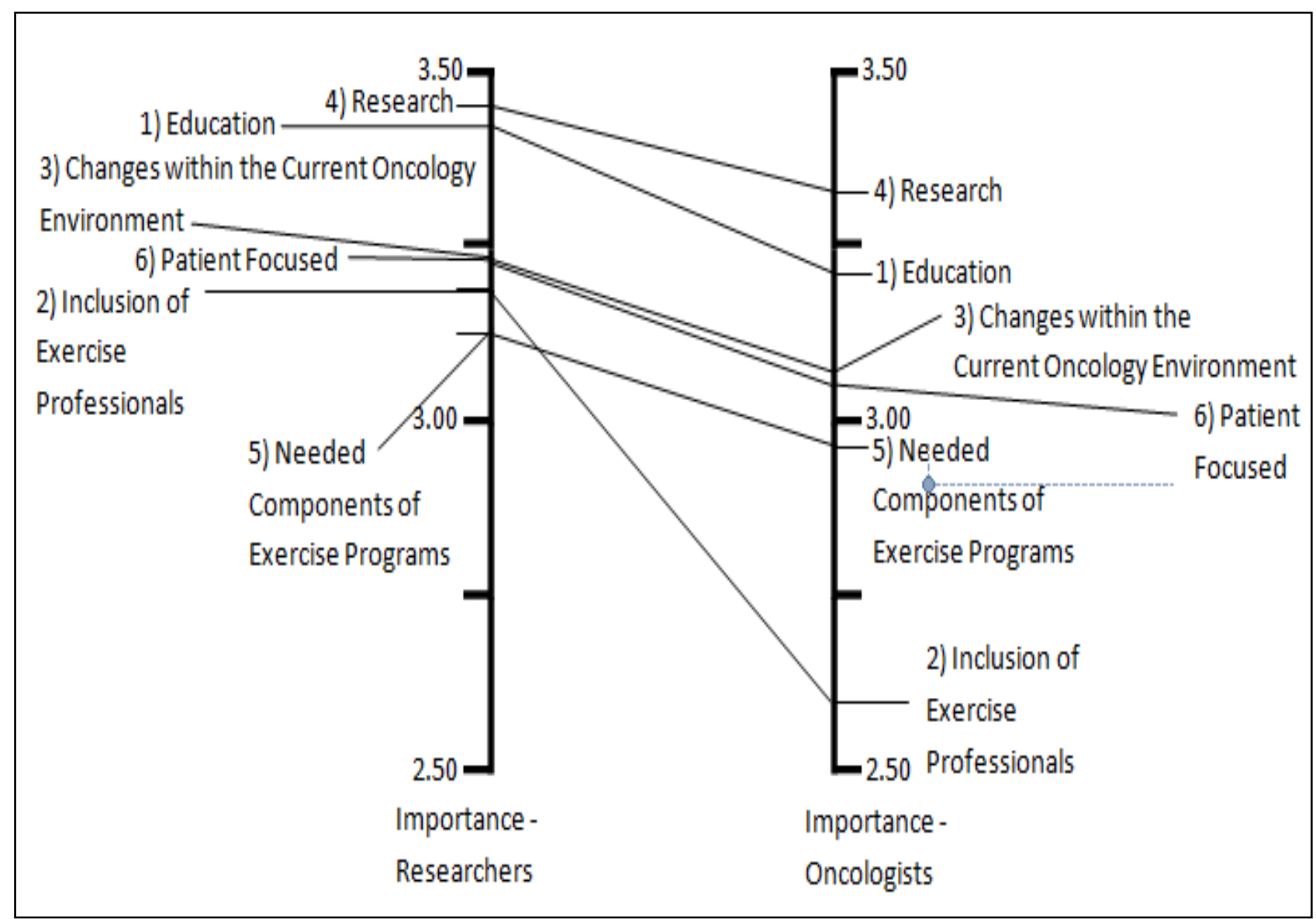

Figure 13. Pattern match display comparing cluster ratings on importance between researchers (the left side) and oncologists (right side). 


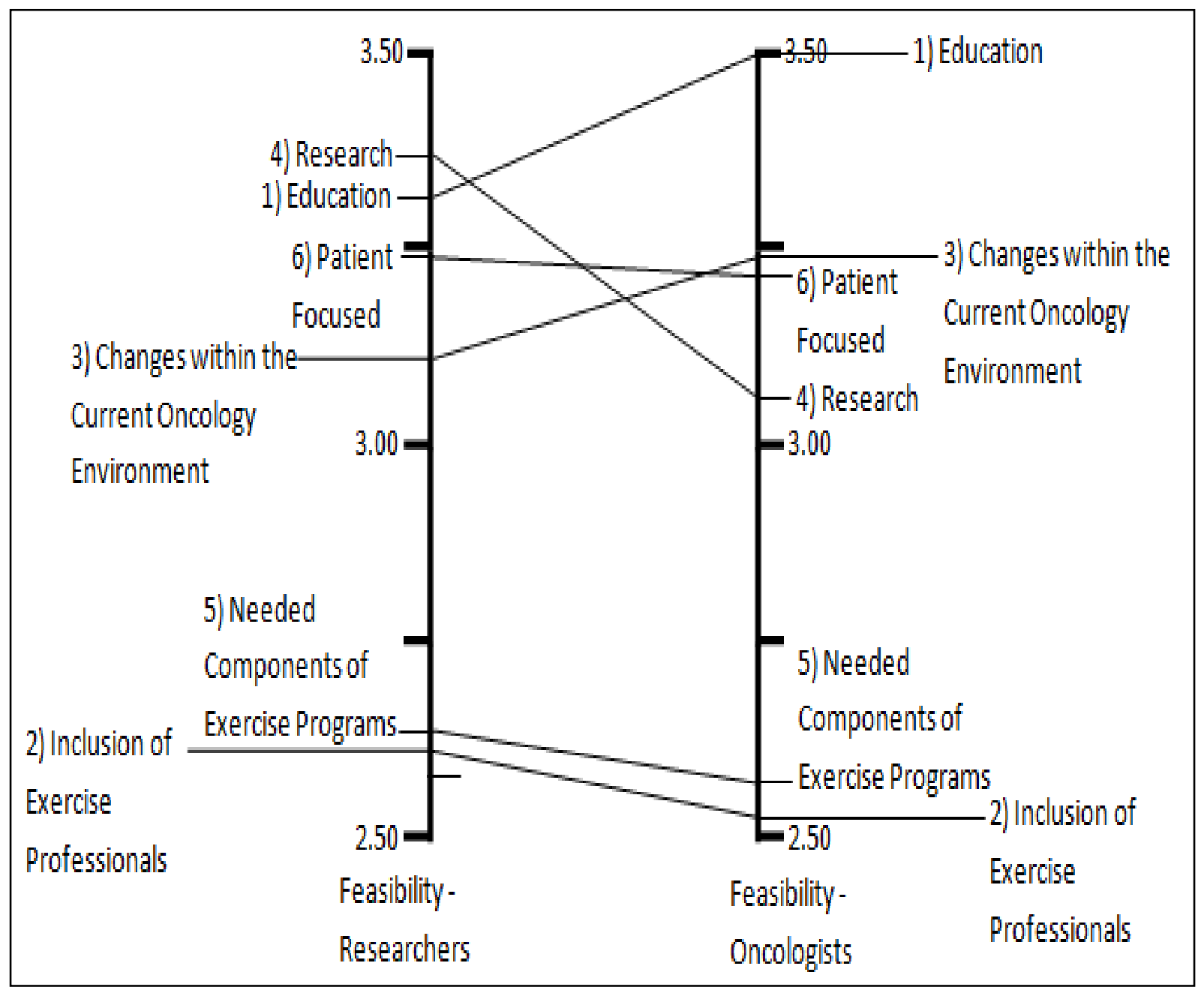

Figure 14. Pattern match display comparing cluster ratings on feasibility between researchers (the left side) and oncologists (right side). 


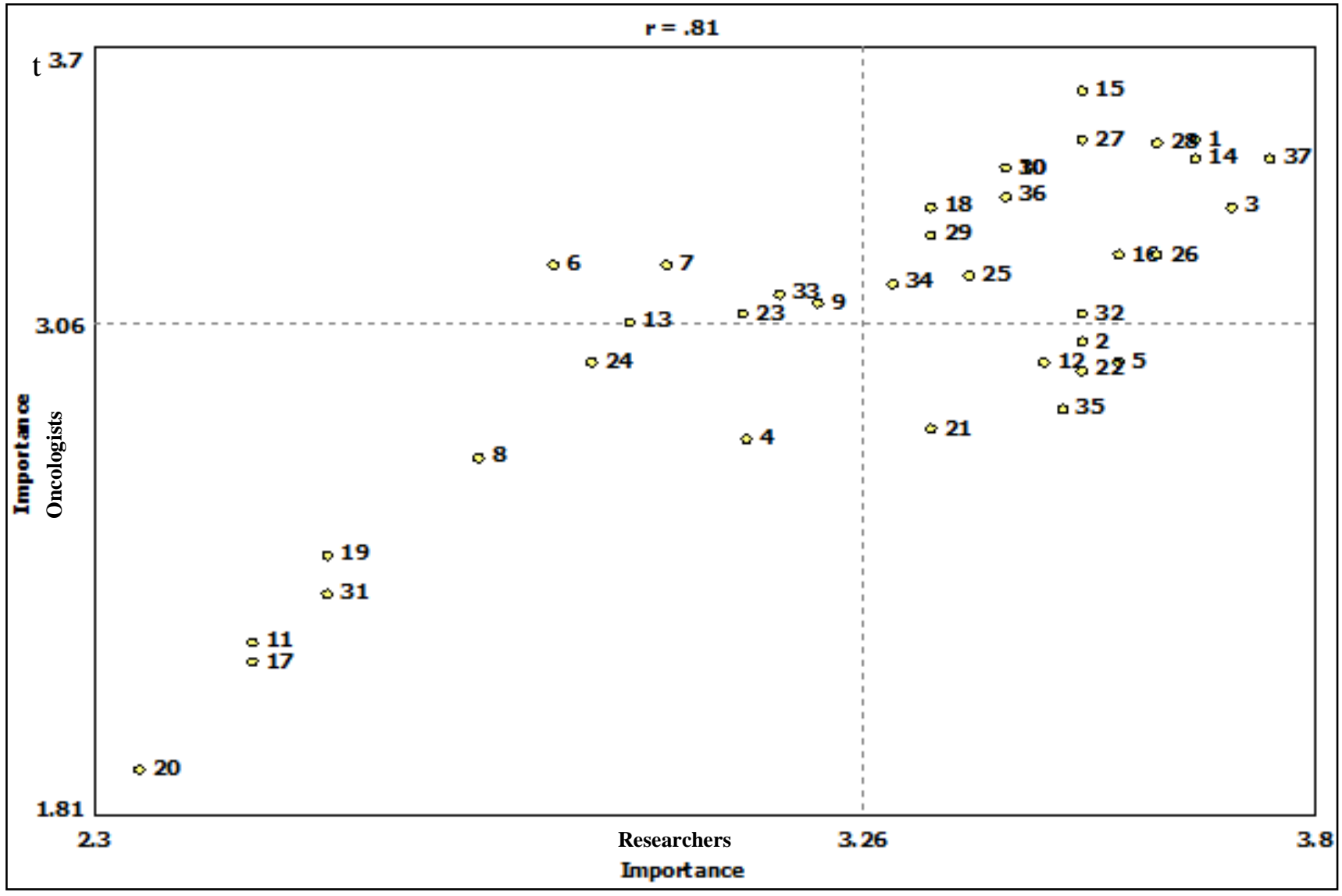

Figure 15. Go-zone display comparing oncologists' importance ratings for statements (y-axis) and researchers' ratings for importance (x-axis). 


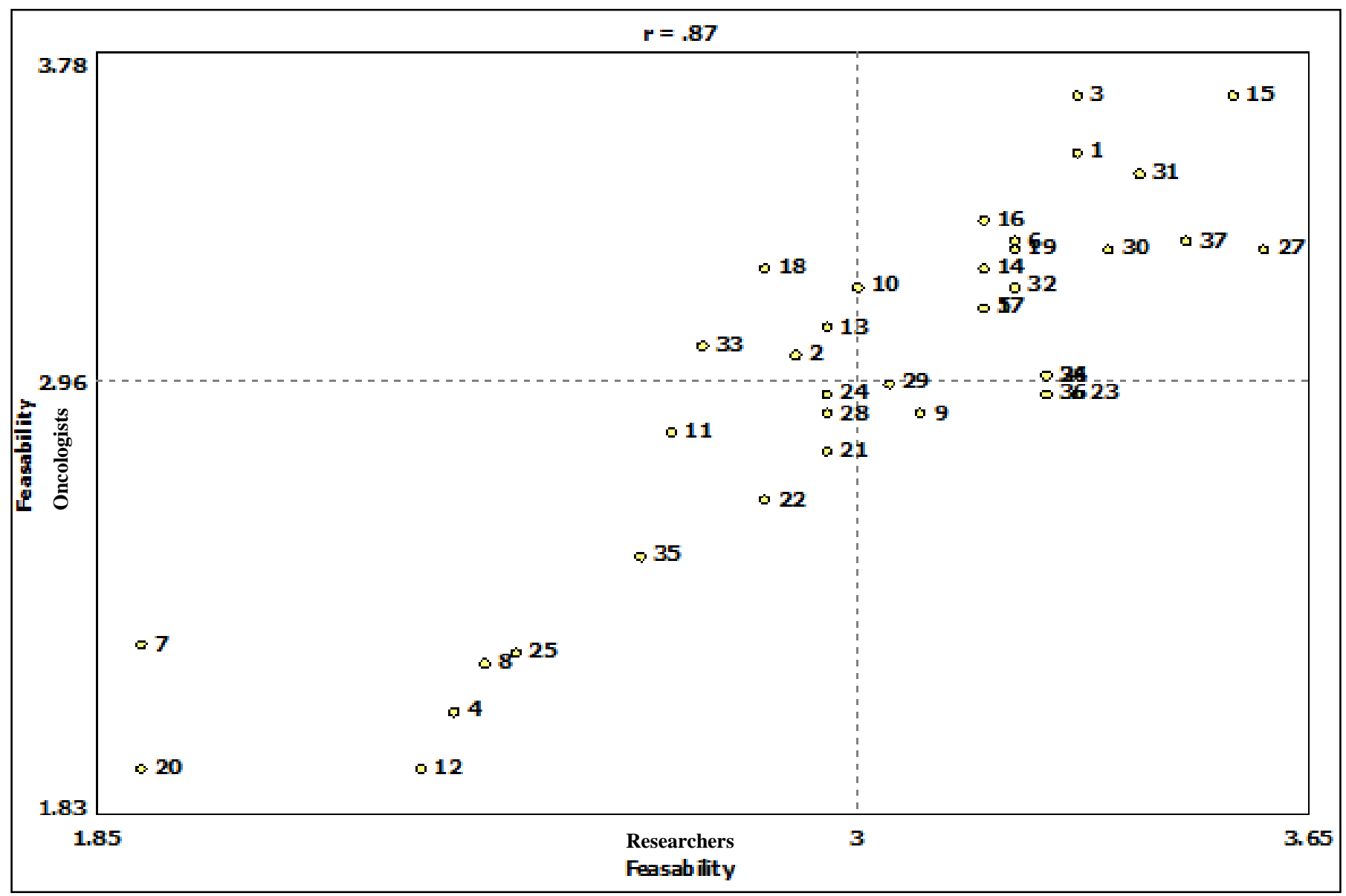

Figure 16. Go-zone display comparing oncologists’ feasibility ratings for statements (y-axis) and researchers' ratings for feasibility (x-axis). 
Appendix A. Brainstorming Demographics - Researchers

1) How many years have you been conducting research regarding physical activity and cancer treatment?

Years

2) What is the approximate number of physical activity and cancer studies that you have been involved with throughout your career?

Studies

3) Which type of cancer do you have the most experience working with?

4) Do you agree that exercise should be part of standard cancer care?

1. Strongly agree $2 . \quad 3.4$ 5. 6. Strongly agree

Please provide a brief response to support your response to the previous question.

5) Moderate physical activities are those, like walking, light bicycling, yoga, or Pilates that cause small increases in breathing or heart rate.

In a usual week how many days do you do 30 or more minutes of moderate physical activity (only count bouts of at least 10 minutes)?

Days:

6) Vigorous physical activities cause large increases in breathing or heart rate, like running, lifting weights, aerobics or Zumba.

Again, in a usual week how many days do you do 20 more minutes of vigorous physical activity (only count bouts of at least 10 minutes)?

Days:

7) What is your current age?

Years:

8) What is your gender?

Male Female 
Appendix B. Brainstorming Demographics - Oncologists

1) What is your primary professional setting?

Community cancer center Academic medical center Other (please specify)

2) How many years have you been practicing oncology?

Years:

3) What type of oncology do you practice?

Surgical Radiation Medical Other (please specify)

4) Which type of cancer do you have the most experience working with?

5) Please provide the breakdown of your professional time spent practicing medicine and working on researcher. *Note: The break down between these two roles should equal 100\% Percentage of time practicing medicine:

Percentage of time conducting research:

6) Do you agree that exercise should be part of standard cancer care?

1. Strongly agree 2. 3. 4. 5. 6. Strongly agree

Please provide a brief response to support your response to the previous question.

7) Have you ever been involved with a research study investigating physical activity with cancer patients?

Yes No

8) Moderate physical activities are those, like walking, light bicycling, yoga, or Pilates that cause small increases in breathing or heart rate.

In a usual week how many days do you do 30 or more minutes of moderate physical activity (only count bouts of at least 10 minutes)?

Days:

9) Vigorous physical activities cause large increases in breathing or heart rate, like running, lifting weights, aerobics or Zumba. 
Again, in a usual week how many days do you do 20 more minutes of vigorous physical activity (only count bouts of at least 10 minutes)?

Days:

10) What is your current age?

Years:

11) What is your gender?

Male Female 


\section{Appendix C. Review of Literature}

This review of literature will focus on physical activity (PA) during cancer treatment and the various outcomes that it may affect. Specifically, this review will discuss studies that examine the effects of PA during treatment as well as after treatment. These sections are surveys of recent relevant work within the realm of PA during and after treatment, they are not exhaustive reviews of one particular cancer; instead, strong methodological studies will be examined regardless of cancer type. A section about the promotion of PA and cancer treatment will also be covered. This section will focus on both patients' views of PA and how it may be best promoted as well as oncologists' views and the effect oncologists may have on their patients' activity levels. Also, a small section on the health disparities of the Appalachian region will be presented. In addition, the use of the concept mapping methodology developed by Kane and Trochim (2007) will be examined through examples from various public health studies. Lastly, this review will end with a short conclusion that will identify gaps in the current literature base as well suggestions for future directions.

\section{Cancer: A Brief Primer}

This section is meant to provide a brief overview of cancer, some of its most common treatments, and other terms and conditions that readers may come across when reading the literature regarding PA and cancer treatment. A more thorough coverage of treatment options, diagnostic procedures, and side-effects of cancer and cancer treatment is beyond the scope of this document. Cancer is an overarching term for any cellular disorder characterized by uncontrolled growth (Lowitz \& Casciato, 2009). Furthermore, cancer cells have many unique characteristics which set them apart from normal cells. Most cancer cells originate from a single abnormal cell that has undergone a mutation which causes it to divide at an increased rate. The causes of these mutations can vary greatly and while some are identifiable (e.g. carcinogens 
causing mutations in the lung cells of a smoker), many are not traceable or understood by modern science. Cancer cells can often reproduce indefinitely, whereas the body’s normal cells are only able to move through a limited number of reproductive cells. In addition, cancer cells, due to defects in their DNA, have an increased ability to survive in different environments, which allows for cancer cells to originate in one part of the body and then move and continue to live and reproduce in another.

When cancer is detected, the treatment pursued will vary greatly depending on the type of cancer, when in its lifecycle the cancer was detected, and the health of the patient. Through there are numerous treatment procedures and strategies, three of the main forms of cancer treatment are surgery, radiation, and chemotherapy (Airley, 2009). Treatment plans will often use all three of these options in conjunction with one another. Various surgical procedures can be utilized to remove cancerous tumors and surrounding tissue as a means of preventing the continued growth and reoccurrence of cancer cells. Radiation treatment is simply the use of radioactive materials (e.g. X-rays or gamma rays) to attack and kill or control cancer cells. Chemotherapy on the other hand, has the same goal, but is done using chemicals. Though advancements have been and continue to be made, there are often a number of side-effects. Potential side-effects of treatment include but are not limited to: fatigue, nausea, hair loss, depression, loss of appetite, weight gain/loss, and loss of physical functioning (National Cancer Institute, 1999). A side-effect that is often associated with breast cancer, but can be a side-effect of other cancers as well, is lymphedema. Lymphedema is swelling due to the blocking of the lymph system (Pub Med, 2011). Around 10-15\% of breast cancer patients will suffer from lymphedema of the arm as a result of the removal of the breast and underarm lymph tissue (mastectomy). 
When a patient is diagnosed with cancer, their cancer is often described in terms of stages. The higher the stage number, the more aggressive the cancer; thus, diagnoses with a higher stage are more likely to spread to other parts of the body and will do so at a faster rate (Airley, 2009). For instance, with breast cancer tumors, staging is done partially by tumor size (e.g. tumors larger than $5 \mathrm{~cm}$ are graded as stage 3 ), as well as other characteristics (e.g. regardless of size, if the tumor has extended into the chest wall it will receive a stage 4 diagnosis). Treatment will vary greatly depending on the stage of the tumor.

\section{Physical Activity during Treatment}

The investigation of PA during cancer treatment has been conducted mostly with an eye towards lessening the effects of the numerous adverse side-effects that often accompany treatment (Courneya \& Friedenreich, 2001). Due to the large number of studies that have been published within the past two decades, researchers have been able to compile systematic reviews and meta-analyses of work done within this area. For instance, in their preparation for developing exercise guidelines for cancer survivors for the American College of Sport Medicine (ACSM), Schmitz and colleagues (2010) reviewed literature examining PA during and after cancer treatment for numerous cancers. Findings of their review were grouped according to the categories used by the National Heart, Lung, and Blood institute to evaluate scientific evidence. Findings that were grouped under category “A” are supported overwhelmingly by data from randomized controlled trials (RCTs). In the "B" category, there are fewer RCTs and/or the studies are small with inconsistent results. Findings are grouped under the "C" Category if they come from non-controlled studies. Finally, findings will be labeled with a " $D$ " if there is insufficient reasoning for their inclusion in groups $\mathrm{A}, \mathrm{B}$, or $\mathrm{C}$. 
According to the panel's findings, only breast cancer had a substantial enough literature base of RCTs to make sound conclusions about the use of PA during treatment (Schmitz et al., 2010). The other cancer types examined did not have their findings separated between during or after treatment. For breast cancer studies conducted with patients undergoing treatment, the following three outcomes fell under the "A" category: safety, aerobic fitness, and muscular strength. The evidence that pointed to the positive effects of PA on body size/composition, fatigue, anxiety, and quality of life (QOL) was judged to be within the "B" category. While the findings for other cancer sites are not as robust, the panel did state: “exercise is safe both during and after most types of cancer treatment...” (Schmitz et al., 2010, p. 1415).

In an updated review of studies investigating PA during treatment (Speck, Courneya, Masse, Duval, \& Schmitz, 2010), which was not separated by cancer type, significant weighted mean effect sizes (WMES; <.3 small effect; .3-.7, medium; >.7, large effect) were found for the following outcomes (findings are reported as: number of studies, WMES, $P$-value): upper body strength (8, 0.39, $p=0.005)$, PA level $(12,0.38, p=0.001)$, aerobic fitness $(17,0.33, p=0.009)$, functional QOL $(4,0.28, p=0.04)$, body fat percentage $(7,-0.25, p=0.04)$, body weight $(8,-$ 0.25, $p=0.05)$, self-esteem $(3,0.25, p=0.02)$, lower body strength $(7,0.24, p=0.006)$ and anxiety $(6,-0.21, p=0.02)$. Besides reporting estimated effect sizes, Speck and colleagues also categorized many of the components of the studies included in their review. The most common cancer type included in studies was breast, which was included in $83 \%$ of the studies they reviewed. The average sample size of the intervention group for projects during treatment was 32 and most interventions lasted between five weeks and 3 months (52\%). Interventions overwhelmingly featured aerobic activity, either alone or combined with other exercise modes (88\%). The majority of studies’ participants participated in three to five days (64\%) of 
moderate to vigorous activity (61\%). The average study lost $12.7 \%$ of participants at follow-up. These reviews paint a brief picture of what is currently known within this field. Both studies highlight the positive effect of PA on physical outcomes such as fitness, strength, and body composition. ACSM's board of experts (Schmitz et al., 2010) found that exercise is safe and tolerable. However, less is known about psychosocial outcomes and neither review reported strong findings for biological variables related to cancer (e.g. immune functioning, blood cell counts etc). There is still a need for studies that examine a wide range of variables, especially for cancer sites other than breast.

Selected controlled trials with multiple outcomes. One of the largest random controlled trials examining the effects of PA during cancer treatment was done with breast cancer patients recruited from three cancer centers throughout Canada (Courneya et al., 2007). For this study, over the course of almost two and half years, just under $1500(N=1468)$ women were assessed for inclusion. Just over half of the women assessed (736) met the eligibility requirements. From these eligible participants, 242 participants (33\% of those eligible, 16.5\% of those assessed) entered the study. The average age of participants was 49.2 and their average beginning weight was 155.65 lbs $(\mathrm{SD}=31.53)$ with a $\mathrm{BMI}$ of $26.6(\mathrm{SD}=5.5)$. All the participants were just beginning their chemotherapy treatment regimens and were followed throughout the duration of their treatments, which on average, lasted 17 weeks $(\mathrm{SD}=4)$. Participants were first stratified by cancer center and chemotherapy regimen and were then randomized between three groups: aerobic training, resistance training, and usual care. Both of the exercise groups partook in supervised exercise three times a week. Members within the aerobic and resistance intervention groups attended $72.0 \%$ and $68.2 \%$ of their exercise sessions respectively. 
The researchers investigated a number of variables, including but not limited to: fitness, both aerobic and anaerobic; body composition; anxiety; QOL; depression; and measures of lymphodemia (Courneya et al., 2007). However, few significant results were found between the intervention groups and the control group. Self-esteem did slightly increase for each of the intervention groups while it decreased for the control group from the beginning of the study to the end; this represented the only significant finding among the patient rated outcomes $(p=0.015$ for the aerobic training group compared to the control and $p=0.018$ for the resistance group). As previous studies have found, the aerobic fitness group increased $\mathrm{VO}_{2}$ max significantly more than the control group and the resistance training group, while the resistance training group significantly increased muscular strength when compared to both the other groups. Though between-group comparisons yielded few significant results, when the aerobic training group was compared with the control group, improvements in aerobic fitness had small correlations with numerous outcome variables, including: QOL ( $r=0.26, p=0.001)$, fatigue $(r=0.25, p=0.002)$, depression $(r=-0.24, p=0.003)$, and anxiety $(r=-0.18, p=0.025)$. Similarly, when the resistance training group was compared with the control group, improvements in lean body mass were associated with the following positive outcomes (once again, all small effects): QOL ( $r=$ $0.19, p=0.022)$, self-efficacy $(r=0.19, p=0.022)$, and depression $(r=-0.19, p=0.019)$. Lastly, neither exercise group had significant changes in arm circumference, a measurement used to signify lymphodema for breast cancer patients. The defined timing of the interventions for participants and the sample size of this study give credence to these limited findings. Quality of life, which was one of the main outcomes, may not have shown between-group differences due to the large variability of QOL scores (the standard deviation was 25). However, even with the methodological strengths of this study, the lack of significant findings cannot be ignored. The 
adherence rates of the two intervention groups were not as high has desired and may have negatively affected changes in numerous outcomes. The secondary tests of the relation between positive physiological exercise outcomes and various psychosocial variables support this hypothesis. Each of the two intervention groups largely met the fitness improvement goals built into their interventions (i.e. a 20\% increase in aerobic intensity over the duration of the aerobic training group) which may point to a need to increase either or both frequency and intensity of PA.

Lowered QOL is a prevalent side-effect of cancer treatment that has been the focus of numerous studies. Fatigue, which may relate and/or be a source of lowered QOL is another common side-effect. Much like the above mentioned study, QOL was one of the two main dependant variables along with fatigue in a large study done with patients undergoing treatment for a diverse set of cancer diagnoses (Adamsen et al., 2009). Both fatigue and QOL were measured using the European Organization for Research and Treatment of Cancer Quality of Life Questionnaire (EORTC QLQ-C30). Using a two-group design, 269 individuals were randomized between an experimental and control group. Participants were mostly female (73\%), had an average age of $47.2(\mathrm{SD}=10.65)$, and were diagnosed an average of 85 days before they entered the study. Individuals in the six-week intervention took part in a group based exercise program consisting of both low and high intensity exercise across different modalities. Participants exercised four days a week for a total of nine hours. Participants receiving the intervention made it to $70.8 \%$ of 24 total exercise appointments. As was to be expected, the intervention group increased their fitness significantly as they had a $10.7 \%$ increase in their $\mathrm{VO}_{2}$ max compared with no change in the control group (note: the mean difference is not reported here due to an error in the original publication resulting in a missing table which 
included the test statistics). In terms of fatigue, while neither group improved pre to post, the intervention group’s fatigue decreased significantly less ( $p=0.02$, effect size $=0.33,95 \%$ CI 0.04 to 0.61 ). Global QOL did not differ between the two groups; however, many measures of well-being from the Medical Outcomes Study Short Form (MOS SF-36) did. Among these were: vitality ( $p<0.0001$, effect size, 0.55 , 95\% CI: 0.27 to 0.82 ), physical functioning ( $p=$ $0.01,0.3,0.09$ to 0.65$)$, and mental health $(p=0.04,0.28,0.02$ to 0.56$)$. Though the effect sizes of this study were small to medium, the short duration of the intervention helps to provide evidence that even in the short-term exercise may be beneficial both physically and psychosocially. The lack of follow-up data after the intervention prevents judgments from being made about the lasting effects of increased PA and if the increased activity levels that often come with interventions are sustained.

In another large study, this time with prostate cancer survivors undergoing treatment, the researchers aimed to better understand the effects of and differences between aerobic and anaerobic exercise (Segal et al., 2009). Over a period of three years, 121 participants were recruited to take part in the study. Individuals were either placed into a resistance training intervention, an aerobic training intervention, or received standard care. The average age of individuals was 66.3 (SD = 7), possessed a BMI of $28.6(12.2)$ and the majority had a Stage II diagnosis (78.5\%). Individuals in either intervention group participated in supervised exercise three days a week for six months. Participants in the resistance intervention completed a median of $88 \%$ of their sessions while aerobic intervention participants completed a median level of $83 \%$ of their sessions. Interestingly, only the resistance training group improved significantly when compared to the control group in terms of fitness improvements. This effect was true for both aerobic measures, such as $\mathrm{VO}_{2} \max (p=0.041)$, and upper and lower body strength $(p<0.001$ 
for both). In terms of fatigue, there was no difference between the groups. However, there was an interaction between group and time $(\mathrm{F}=3.84 ; p=0.005)$. Post hoc analysis showed that the resistance intervention group improved their fatigue throughout and was the only the group to do so $(p=0.02$, mean change $=0.20,95 \% \mathrm{CI}=0.13$ to 4.53$)$, while the aerobic group only improved their fatigue from baseline to the mid-point (three months). Though the resistance group's improvement in fatigue was not great, the usual care group experienced greater amounts of fatigue at the end of the study than at the beginning which may point to resistance training being an effective intervention to combat the increased fatigue that comes with treatment. In addition, improvement in fatigue were related to improvements in upper body strength $(r=.21, p$ $=0.03$ ) but no other fitness improvements and/or biological markers. While it is disappointing that an intervention of this length that had such a high rate of adherence was unable to produce more significant effects, this study may help lay the ground work for the promotion of strength training during prostate cancer treatment.

A study by Mutrie and colleagues (2007) also examined QOL, with a sample of British breast cancer patients undergoing either chemotherapy or radiation. On average, the women who participated had been diagnosed 162 (SD = 73.8) days prior to joining the study. This study aimed to study the effects of a group exercise program on not only QOL, but also depression, affect, and fitness. Just over 300 women agreed to be screened for the study, of which 203 were randomized between a 12-week exercise program and a usual care control group. Members within the intervention group ( $n=99)$ were encouraged to visit one of the offered classes twice a week and workout on their own at least once. Fourteen classes were offered each week at eight different exercise facilities. Each class consisted of 45 minutes of PA as well as a themecentered discussion (e.g. health benefits of exercise, self efficacy). There were a total of six 
themes; each theme was discussed twice throughout the 12 weeks. The 201 women enrolled within this study on average were 51.6 years old $(\mathrm{SD}=9.5)$, had a BMI of $27.4(5.6)$ and most were undergoing both radiation and chemotherapy treatment (64.2\%).

At the end of the 12-week study, when compared with the control group, women within the intervention group possessed positive differences on a number of outcomes (Mutrie et al., 2007). Their scores on a breast cancer specific sub-scale of the functional assessment of cancer therapy-general (FACT-G) increased at a higher rate (mean change $=2.5$, 95\% CI 1.0 to 3.9, $p$ $=0.0007)$. Six months after the study, the intervention group's scores remained higher $(1.5,0.1$ to $2.9, p=0.039$ ). In addition to seeing a greater increase in the breast cancer specific subscale of the FACT-G, women in the intervention group also saw greater increases at the end of the 12 week study in positive affect $(4.0,1.8$ to $6.3, p=0.0005)$, leisure time PA (minutes; 120 , 83 to $176, p<0.0001)$, shoulder mobility (2.6, 1.6 to 3.7, $p<0.0001)$, and the average distance (m) that they could walk in 12 minutes (129, 83 to 176, $p<0.0001)$. All of these changes remained when measured six months after the study’s completion with the exception of leisure time PA. In addition, throughout the duration of the study, women in the intervention group reported spending fewer nights in the hospital $(p=0.044)$ and fewer visits to their general practitioner $(p$ $=0.011)$.

This study (Mutrie et al., 2007) adds to the literature base in a number of ways. Though there was no difference QOL as measured by general instrument, when using a breast cancer specific QOL measure, women in the intervention group had higher amounts of QOL both following the 12-week intervention and six months later. This finding, while perhaps specific to breast cancer patients, shows the potentially lasting positive effects of PA. These effects were seen with a relatively light amount of PA (supervised exercise twice a week for a total of 90 
mintues, plus one independent bout of PA) which is important as many patients undergoing treatment may not be able to accumulate large amounts of PA. This study also included a novel outcome: visits to the hospital and general practitioner. More research is needed, but it is promising that perhaps increased PA has measureable effects on the amount of care that patients require. Though many positive findings were realized with this study, there are a few components of the study that need to be mentioned. First and foremost adherence to the intervention was not reported. The only PA reported was done so using self-report data (leisure time PA measured utilizing the Scottish PA questionnaire). However, there were significant fitness differences (i.e. 12 minute walk test and shoulder mobility) between the two groups at both 12 weeks and six months which may point to the intervention group truly becoming more active. Though a more thorough measuring and reporting of PA would help further knowledge about the dose/response relationship between PA and many variables, this study does highlight many physical and psychosocial benefits of increased PA during cancer treatment.

The few studies discussed in this section highlight a few of the numerous studies that have been conducted throughout the better part of the past decade. These studies are examples of many of the newer studies which possess solid designs (i.e. randomized assignment with a control group). However, as the results of these studies showed, findings are still mixed. More work is needed in the coming years to better understand the potential short term and long term effects of PA during cancer treatment.

\section{Physical Activity after Treatment}

Many of the same outcome variables as those mentioned above have been examined with patients who have finished treatment. Many other outcomes have been studied as well. For instance, through retroactive observational studies, researchers have studied the effect of PA on 
survival (e.g. Holick et al., 2008) as well as cancer recurrence (e.g. Sternfeld et al., 2009).

Though treatment has become more effective, unfortunately many treatments still have prolonged effects. In addition, many cancer diagnoses are related to cancer recurrences. These potentially long lasting effects as well the strong tendency for cancer patients to both put on weight and become inactive point to the time after treatment as a needed time-point for PA promotion.

Though few large reviews or meta-analyses have been done solely involved patients after active cancer treatment, some of the aforementioned reviews have examined this time period as well. In the ACSM's (Schmitz et al., 2010) roundtable, researchers examined the effects of PA after treatment on a number of outcomes for breast cancer survivors (once again the researchers believed that other cancers did not possess a rich enough literature base of after treatment studies to make any conclusions). Once again, using the National Heart, Lung, and Blood Institute’s rating system, through the examination of 32 studies, the following outcomes were found to be in evidence category “A”: safety, aerobic fitness, muscular strength, flexibility, physical function, and safety regarding lymphedema. Within the "B" category resided the following outcomes: body size, body composition, QOL, fatigue, depression, anxiety, and body image. The outcomes rated within the "A" category are outcomes that would be expected within the "normal" population (e.g. strength, aerobic fitness, and flexibility), suggesting that cancer survivors can derive the same benefits from exercise as an individual never diagnosed with cancer. Evidence category "B" possesses psychosocial variables that were not found in any of the categories for the during-treatment time period. Even after treatment, cancer survivors are at an elevated risk for mental health issues such as anxiety, lowered QOL, and depression. Physical activity may be a way for individuals to "treat" or prevent these conditions. 
Speck and colleagues (2010) also examined the after-treatment time period in their review. A number of outcomes were examined, and many positive relationships were found. Outcomes where positive, significant findings were observed include (findings are reported as: number of studies, WMES, $P$-value): fatigue $(14,-0.54, p=0.003)$, physical activity level (16, 0.38, $p=0.0001)$, aerobic fitness $(14,0.32, p=0.03)$, overall QOL $(16,0.29, p=0.03)$, body weight $(14,-0.18, p=0.004)$, and BMI $(16,-0.14, p=0.002)$. Once again these outcomes mirror the outcomes associated with PA in general. Of note, is that in this review, few physical variables related to cancer diagnoses and subsequent treatments, such as bone mineral density (which often decreases), insulin (has been found to be related to certain cancer types), and hemoglobin (often decreases) were found to have an insufficient literature base to produce solid evidence. These variables are examples of variables that need to be studied further and be examples of the findings that may be desired by oncologists and other medical professionals. The cancer diagnosis itself has been thought of as a "teachable moment” (McBride, Clipp, Peterson, Lipkus, Demark-Wahnefried, 2000), however, the end of treatment may be just as an important time to deliver health messages.

Selected controlled trials with multiple outcomes. As the two above reviews begin to indicate, PA after treatment may offer many physical and psychological benefits. Believing that PA after treatment is a beneficial behavior, Pinto, Rabin, and Dunsiger (2009) sought to better understand what variables may predict adherence to a home-based PA intervention. Adherence was measured as both a continuous variable: minutes per week of exercise and pedometer data, as well as a dichotomous variable: whether or not individuals were reaching their own PA goals. To examine what may be related to these classifications of adherence, 43 women were enrolled in a 12-week walking intervention that also included weekly counseling sessions delivered 
telephonically. A number of demographic variables as well as other variables were measured at the onset and then examined retroactively to "predict” adherence.

Overall, PA increased greatly for the 43 women from week 1 to week 12 (Pinto et al., 2009). Participants increased their average minutes of exercise from $43.12(S D=44.32)$ to 128.53 (76.82) as well as their pedometer steps from 4471.70 (5196.10) to 14571.47 (9489.48). The results in terms of the dichotomous definition of adherence as meeting weekly goals were not as bright. The percentage of women meeting their goals decreased over this time period from $88.37 \%$ to $55.81 \%$. In terms of predicting adherence, with the exception of baseline PA

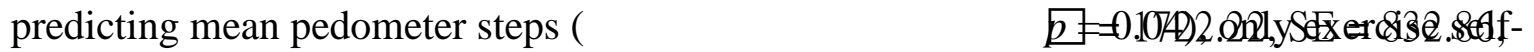
efficacy was related to any of the variables used for adherence; it was significantly related to all

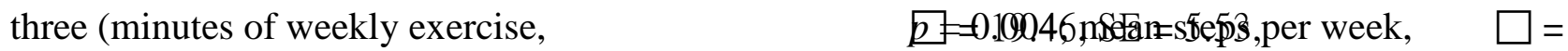
2636.91, $\mathrm{SE}=768.34, p=0.0006$, and exercise goals, $\quad \mathrm{E} 0.48, \mathrm{SE}=0.22, p=0.03)$. Though this study was unable to robustly provide information related to its main purpose of predicting PA levels among cancer survivors, it is promising that on average the women in the study increased their PA significantly. Also, the relationship between exercise self-efficacy and PA should not be overlooked. These results may point once again to the time after treatment as an ideal intervention point. At this time it may prove to be prudent to provide patients with information about the effects of PA and educate them on how to achieve PA to potentially increase efficacy.

As the reviews in the earlier portion of this section found, cancer survivors can make positive changes in their fitness levels. One study that echoes this finding was done by Matthews and colleagues (2007). The researchers involved with this study set out to examine the effectiveness of a home-based walking intervention in breast cancer survivors. Participants were 
given pedometers and had weekly step goals. In addition, they had an initial counseling session (30 min) as well as five follow-ups (10-15 min). The program lasted a total of 12-weeks. A total of 22 women were assigned to the intervention group while 14 women were enrolled in a waitlist control group. The women in the study had an average age of 53.5 and a BMI of 29.1.

The results of this study indicated that the intervention was moderately successful in increased PA. Most of the women reached their weekly walking goals throughout the study (94\%). In addition, those in the intervention group increased their walking time by just under 12 minutes (11.9), which was significantly ( $p=0.01)$ more than the control group $(1.7$ minutes). Predictably, these results were similar in terms of steps per day. The intervention group increased their daily steps by an average of $1152.5(\mathrm{SD}=2408.8)$ compared $(p=0.04)$ to a decrease of $559.1(\mathrm{SD}=1326.5)$ in the control group. In terms of physical measurements such as weight and BMI, there were no significant between group differences. This result may indicate that while the intervention was successful in raising daily step counts, walking alone was not enough PA to make significant bodily changes. Though this study's relatively small number of participants makes it difficult to extrapolate the findings, it is promising that the intervention had high adherence rates and was successful in its goal: raising PA. Future studies are needed to better understand how intense of an intervention cancer survivors will adhere to and if more intense levels of PA are needed to make significant changes to weight, BMI, or other physical outcomes.

Quality of life represents one of the most often examined variables for studies done during and after cancer treatment. Quality of life represents one main variable in a novel study run by Daley and colleagues (2007). The research team was worried that perhaps it was the added attention that intervention groups received compared to control groups in PA studies done 
with cancer survivors that attributes for positive changes. To address this concern, a three-armed design was utilized. A total of 108 breast cancer survivors were randomized to three groups: a usual care control group, an exercise group, and an exercise-placebo group. Both the exercise group and the exercise-placebo group attended thrice weekly one-on-one meetings with exercise specialists for eight weeks. However, those in the placebo group only did light stretching.

It was found that the exercise group experienced numerous positive benefits when compared to the control group (Daley et al., 2007). However, the exercise-placebo group also differed from the control group at the end of the intervention on the breast cancer specific subscale of the FACT-G (Mean difference $=9.57, \mathrm{CI}=0.00$ to $19.10, p=0.049)$, depression, $(-$ 5.66, -9.76 to $-1.55, p=0.001)$ and aerobic fitness $(2.25,0.22$ to $4.28, p=0.021)$. The exercise group also differed from the control group on these variables (FACT-B: 13.14, 3.44 to 22.84, $p=$ 0.02; depression: $-6.01,-10.21$ to $-1.81, p=0.001$; aerobic fitness $2.89,0.78$ to $4.99, p=0.002$ ). In addition to possessing greater differences compared to the control group than the exerciseplacebo group, those in the exercise group also saw improvement in overall QOL (9.80, 2.20 to 17.40, $p=0.004)$. Follow-up was done at six months, at this time depression was the only variable on which the exercise group significantly differed from the control group (-4.49, -8.78 to $-0.20, p=0.35)$. Interestingly, the exercise-placebo group also differed from the control group on depression at this time point, and differed more than the exercise group $(-4.98,-9.14$ to -0.81 , $p=0.009$ ). These findings highlight the potential powerful tool that PA can be With regard to QOL. In addition, the positive improvements that the exercise-placebo group experienced during the intervention may show that even light exercise (in this case, stretching) can have benefits. The relationship between QOL and higher amounts of PA that this study highlighted is not well understood and represents an area that needs further exploration. 
Another study utilizing novel methods also examined the effect of PA on QOL (Milne, Wallman, Gordon, \& Courneya, 2008). The study was run in Australia with breast cancer survivors ( $n=58$ ) who had completed all forms of treatment except for hormone treatment. The intervention utilized in the study was a 12 week exercise program. Three times a week participants would visit a rehabilitation clinic where they took part in supervised exercise consisting of both anaerobic and aerobic exercises. This study did not randomize participants between an intervention and true control, instead, the study lasted 24 weeks and women were randomized between two groups: a group that did the intervention during weeks one to 12, and a group that participated in the intervention between weeks 13 and 24 . When participants were not participating in the exercise program they received phone calls every three weeks to maintain their interest, however, they were not given any specific instructions regarding PA during these phone calls. These two groups were compared on a number of variables: QOL; fatigue; social physique anxiety; fitness; and physical, social, and emotional well-being. The women enrolled in the study had an average age of $55.1(\mathrm{SD}=8.20)$, a BMI of $26.3(\mathrm{SD}=4.6)$, an average treatment length of just under 170 days $(169.5, \mathrm{SD}=47.5)$, and had been done with treatment for 13 months $(\mathrm{SD}=3.97)$.

Researchers found moderate levels of adherence to the exercise intervention in both groups (Milne et al., 2008). On average, women in the immediate exercise group (IEG) attended $60.4 \%$ of their sessions (21.7 of 36 sessions), while those in the delayed exercise group (DEG) attended a similar number (62.2\%, 22.4 of 36 sessions). The two groups were compared on the above mentioned variables throughout the study (data were collected every six weeks). Using a two (group) by five (time: baseline and then every six weeks thereafter) repeated measures analysis of variances tests (RM-ANOVA), the researchers were able to determine if there was an 
interaction between time and group placement. Significant interactions were found for QOL ( $F$ $=21.8, p<0.001)$, and fatigue $(F=8.8, p<0.001)$. For both measures, at every time-point, the IEG scores signified better outcomes than the DEG. Significant increases in QOL were seen for the IEG from baseline to week six (mean change $=12.6,95 \% \mathrm{CI}=7.8$ to $17.4, p<0.001$ ), from week six to 12 (8.2, 4.8 to $11.5, p(0.001)$, and week 18 to $24(4.9,2.0$ to $7.8, p<0.001)$. The IEG's QOL scores were higher than the DEG's when measured at week 6 (mean group difference $=17.9,95 \% \mathrm{CI}=11.8$ to $24.0, p<0.001)$ and week $12(28.6,22.0$ to 35.1, $p<0.001)$. The DEG experienced a significant decrease in QOL from week six to week 12 (mean change = 2.3, 95\% CI $=-4.5$ to $-0.2, p=0.034)$ then significant increases from week 12 to $18(27.7,22.6$ to $32.8, p<0.001)$ and from week 18 to week $24(4.8,1.4$ to $6.6, p=0.003)$. In terms of fatigue, the IEG experienced significantly less fatigue than the DEG at both week six (mean group difference $=-3.9,95 \% \mathrm{CI}=-1.8$ to $-6.0, p<0.001)$ and week $12(-5.4,-3.3$ to $-7.6, p<0.001)$; at no time did the DEG possess significantly lower amount of fatigue. However, those in the DEG did see their fatigue scores decrease (signifying less fatigue) from week 12 to 18 (mean change $=$ $-6.3,95 \% \mathrm{CI}=-8.2$, to $-4.3, p<0.001)$.

When physical fitness was examined from baseline to the end of the 24 weeks, significant improvements were seen for both groups for aerobic fitness (IEG, $t(28)=3.5, p=0.002$; DEG, $t(28)=2.2, p=0.034$ ) (Milne et al., 2008). Significant gains were also seen for muscular strength as measured by bicep curls (IEG, $t(28)=12.9, p<0.001$; DEG, $t(28)=10.1, p<0.001$ ), leg press (IEG, $t(28)=12.2, p<0.001$; DEG, $t(28)=13.9, p<0.001)$ and chest extension (IEG, $t(28)=9.0, p<0.001$; DEG, $t(28)=18.3, p<0.001)$. The design of this study showed that exercise can cause significant improvements for a number of variables of concern to cancer survivors, namely fatigue and QOL. These improvements seem to be maintained at least for a 
period of three months as the improvements experienced by the IEG were sustained when their exercise program ended. While this study’s novel methodology produced interesting and useful results, there are some inherent weaknesses. The lack of true control group makes it difficult to understand the true effects of time and PA. The lack of PA data during the time periods when groups were not participating in the intervention is concerning. The IEG may have sustained activity once their exercise program. In addition, follow-up data of both groups would add greatly to the findings. If the DEG experienced the same sustained benefits after their program ended and if the IEG sustained their improvements over a longer duration of time these results would add value to the argument for the inclusion of supervised exercise at the conclusion of active cancer treatment.

As many of the above studies have shown, exercise during or after treatment can have many beneficial outcomes. Among these outcomes, as will be discussed below, is increased survivorship. The biological mechanisms that take place as a result of increased PA for cancer survivors that lead to decreased mortality are not well understood. It has been hypothesized that insulin levels may have a role in breast cancer prognosis (Goodwin et al., 2002). To examine if exercise interventions could cause decreases in insulin levels breast cancer patients, Ligibel et al. (2008) designed a study to test the effects of a 16-week exercise intervention on, among other variables, insulin levels. A total of 101 women who had completed treatment at least three months prior to the study were randomized between a control group $(n=50)$ and an intervention group ( $n=51)$. On average, women within the study were $52.5(\mathrm{SD}=9)$ years old and most had received chemotherapy (72\%). The intervention employed was a mix of supervised and home based exercise as well as strength training and aerobic training. Throughout the 16 weeks participants attended twice weekly supervised exercise sessions where they did 50 minutes of 
strength training. Participants were also instructed to complete 90 minutes of aerobic activity each week. Insulin and glucose measurements were done for both groups pre and post, anthropometric measurements were taken at these two time points as well. However, only the intervention group was tested for any fitness measures; participants in this group increased their strength on the different exercises included in the intervention between $42 \%$ and $106 \%$, they also increased their weekly PA by 11 minutes over the 16 weeks. Participants in the intervention group attended $73 \%$ of their weekly strength sessions and completed an average of 114 minutes of aerobic exercise each week. The only physical measurement for the exercise group which saw a significant decrease when compared to the control group was hip circumference which lessened by $2.3 \mathrm{~cm}(p=0.02)$.

Insulin levels were the main focus of this study (Ligibel et al., 2008). The exercise group significantly ( $p=0.03$ ) decreased their insulin levels by $2.86 \mu \mathrm{U} / \mathrm{mL}$ compared to an insignificant drop of $0.27 \mu \mathrm{U} / \mathrm{mL}$ in the control group (the between group difference was also insignificant, $p=0.07)$. The findings from this study are somewhat mixed as insulin levels did drop in the exercise group, but not significantly when compared to the control group. In addition, no other physical differences were seen with the exception of hip circumference. This may be a result of strength training's ability to decrease insulin levels and not the effect of weight loss or bodily changes. More intense or a higher accumulation of aerobic activity may be needed to see significant decreases in weight, BMI, or fat mass. If these numbers can be improved it is likely that insulin levels may drop at larger levels as increased levels of insulin are related to being overweight/obese. Though the findings of this study are not overwhelming, this study does highlight the potential of exercise interventions to lessen insulin levels which may increase survival rates of breast cancer survivors. 
Using observational data, researchers have examined the effects of PA on survival after a cancer diagnosis. Once again, most studies within this realm have been done with breast cancer survivors. The following three articles that will be discussed all examined breast cancer patients. The Life After Cancer Epidemiology (LACE) study is an ongoing large scale study of women diagnosed with early stage breast cancer that hopes to better understand the role that behavioral factors such as PA and diet have on health outcomes (Sternfeld et al., 2009). For this particular study, 1970 women who had filled out the PA questionnaire following treatment within the LACE study were examined. The PA questionnaire assessed the following domains of PA: occupational, transportation, recreational, and non-work routines. Each domain was divided into activities (e.g. dancing for recreational) and women were asked to mark down any activity and they participated in at least once a month over the past six months as well as provide the duration and frequency of the activity. Standard metabolic equivalent (MET) values were assigned to each activity, frequency of activity was then multiplied by duration; these values were then added from all the activities from which a weekly average of MET hour (MET-h/wk) was computed. All the women in this study had been diagnosed with breast cancer between 1997 and 2000, filled out the survey within 39 months of their diagnosis and had no documented cancer recurrence. The women were recruited from northern California (82\%), Utah (12\%) or were part of a previous study (6\%). To track participants' health, health status surveys were sent out semiannually until April 2006. If any adverse health condition was reported, follow-up was done by phone. Nonrespondents were also telephoned. If participants could not be reached by survey or phone a mortality search was done and if a death had occurred, death was confirmed by death certificate. Cox proportional hazard model, with time since diagnosis as the time scale were 
used to estimate risk for each of the different outcomes (e.g. cancer recurrence, breast cancer mortality).

A total of 225 (11.4\%) women within the study had a recurrence of their breast cancer, another 102 (5.2\%) had passed away due to breast cancer, while another 187 (9.5\%) were deceased due to other causes (Sternfeld et al., 2009). In terms of PA, overall, participants reported fairly high amounts. The median MET-h/wk was 44.3 (IQR 29.3-62.3). Risk of cancer recurrence reduced for women who did some activity compared to those who did not. However, risk did not continue to significantly lower as activity levels increased. A similar trend was apparent for cancer mortality. When all-cause mortality was examined, there was a doseresponse relationship as those who increased in greater amounts of activity had a less risk of mortality (though this was not the case with vigorous activity). When adjusted for age, when compared with women who accumulated less than hour of moderate activity, women who achieved between one and three MET-h/wk had a 41\% reduction of risk for all cause death (hazard ratio $=0.59,95 \% \mathrm{CI}=0.40$ to 0.87 ), those who had an average MET-h/wk between three and six had a $43 \%$ less chance $(0.57,0.39$ to 0.84$)$, while those who averaged six or more MET-h/wk had a 49\% less chance $(0.51,0.34-0.79$, overall $p$ for trend $=0.001)$. These findings suggest that just as with a cancer free population, PA is related to a decreased risk of death. However, it is impossible to tease out the effect of PA alone. Other factors, such as weight, preexisting factors, etc, could play a role. In addition, it is impossible to establish a casual relationship as decreased PA may have been a symptom of disease or worsened health. This study enrolled a very diverse set of women which adds to its findings. The inclusion of multiple domains of PA is another strength. However, as is often the case with large-scale observational 
studies, PA was self-reported which may at least partially explain the high amount of PA reported.

A study done prior to the before mentioned Sternfeld et al. (2009) study utilized one of the U.S.’s largest cohort of individuals whose health behaviors are studied: the Nurses' Health Study (Holmes, Chen, Feshanich, Kroenke, \& Colditz, 2005). The Nurses’ Health Study was established in 1976 with 121700 female nurses across the U.S. These nurses were sent a questionnaire assessing various risk factors for cancer and cardiovascular disease. Follow up surveys were sent every two years until 2004. For the current study, women $(n=2987)$ who self-reported a diagnosis of breast cancer (diagnosis was verified through medical records by physicians who were blinded to the diagnosis) between 1984 and 1998 were included (certain exclusion criteria were also in place, such as previous cancers or a metastatic diagnosis). The dates were chosen because PA was first assessed in 1986 and the researchers wanted to assess PA at least two years after diagnosis. Physical activity was assessed in much the same fashion as described above for the Sternfeld et al. (2009) study (i.e. activities assigned MET values then added together), though the only domain examined was leisure time PA. Among the 2987 women examined, there were 463 (15.5\%) deaths, 280 of which were caused by breast cancer (9.4\%), there were also 370 (12.4\%) breast cancer recurrences. Physical activity was assessed a median of 38 months after diagnosis.

Similar statistical tests (i.e. Cox Hazard proportions) to the prior mentioned study (Sternfeld et al., 2009) were run for total deaths, breast cancer deaths, and recurrence (Holmes et al., 2005). For each outcome, using a multivariable-adjusted (e.g. age, smoking status, BMI) relative risk $(\mathrm{RR})$, there was a significant trend for risk to decrease across all the measured variables as PA increased (total deaths, $p=0.003$; breast cancer deaths, $p=0.004$; and 
recurrence 0.05). For breast cancer deaths, the greatest reduction (50\% less likely than women who had less than three MET-h/wk) in risk was experienced by women who averaged between nine and 14.9 MET-h/wk $(\mathrm{RR}=0.50,95 \% \mathrm{CI}=0.31$ to 0.82$)$. Women who achieved less than nine but more than three MET-h/wk, when compared with those who had less than three METh/wk, had a $20 \%$ risk reduction $(0.80,0.60$ to 1.06$)$. Similar trends were also evident for recurrence, as those classified as achieving between nine and 14.9 MET-h/wk had the greatest reduction. Achieving this amount of MET-h/wk is comparable to walking three to five hours a week at an average pace. This result is a potentially important finding as even relatively small amounts of PA may significantly decrease a breast cancer survivor's risk of death/recurrence. Many of the same limitations of other large scale observational studies, such as self-reported PA and the inability to make inferences about the relationship between activity levels and outcomes do exist. However, these findings do support the further exploration of the potential benefits of PA after a breast cancer diagnosis.

In another similar study, women from Wisconsin, Massachusetts, and New Hampshire diagnosed with breast cancer between 1988 and $2001(n=4482)$ made up the sample of a study (Holick et al., 2008) that found similar results to the study just discussed utilizing the Nurses’ Health Study (Holmes et al., 2005). The methods of this study were similar to the previous two studies_-women diagnosed with breast cancer reported the amount of time they participated in different activities, these results were then transformed into MET-h/wk and used to compare women on different outcomes. Only mortality was examined in this study, both total deaths $(n=$ 412, 9.2\%) and breast cancer deaths $(n=108.2 .4 \%)$. Once again, as total recreational PA increased, risk for death from any cause $(p<0.001)$ and death from breast cancer $(p=0.01)$ decreased. However, with this study, risk continually decreased as PA levels increased, though 
this may be because of the large windows of MET-h/wk for the groupings. For instance, when compared to individuals who had less than 2.8 MET-h/wk, those between 2.8 and 7.9 MET-h/wk had a $38 \%$ reduction (hazard ration $=0.62,95 \% \mathrm{CI}=0.37$ to 1.03 ), those between eight and 20.9 MET-h/wk had a 47\% reduction $(0.57,0.31$ to 0.88$)$, while those greater than $21 \mathrm{MET}$-h/wk had a $64 \%$ reduction $(0.44,0.25$ to 0.76$)$. Once again, these findings, even when the inherent limitations that accompany this method of investigation are considered, build a strong a case for the promotion of PA after treatment.

\section{Physical Activity Promotion with Cancer Patients}

Physicians and other health care professionals often have a very influential role in the promotion of physical activity (PA) with their patients (e.g. Greenlund et al., 2002; Loureiro \& Nayga, 2006). This positive effect on patients' health behaviors may also be true of the effect that oncologists can have on their patients’ activity levels (Jones \& Courneya, 2002b).

Several studies have been done to examine oncologists’ views towards the usefulness of exercise during and after cancer treatment. One of the largest studies to date surveyed 281 practicing medical and radiation oncologists in Canada (Jones, Courneya, Peddle, \& Mackey, 2005). Surveys were sent out to all oncologists in Canada’s largest provinces, which accounted for over $90 \%$ of all practicing Canadian oncologists. Participating oncologists $(n=659)$ were surveyed using an instrument containing open and closed items. Closed items were done using seven point Likert-type scales created by the research team about oncologists’ attitudes towards exercise for patients undergoing treatment and their attitudes towards recommending exercise to these patients. The scales had bookend values of $1=$ strongly disagree, $7=$ strongly agree, and study created ranges and labels of 1-2 = disagree, 3-5 = neutral, and 6-7 = agree. It was found that of those oncologists surveyed, the majority believed exercise was beneficial (62\%, $\mathrm{M}=5.7$, 
$\mathrm{SD}=1.2)$, important $(55.8 \%, 5.6,1.2)$ and safe $(63.1 \%, 5.6,1.2)$. Interestingly, many oncologists (73.2\%) did not think that other oncologists believed patients should exercise $(\mathrm{M}=$ 4.0, SD = 1.3), and while as previously mentioned, they believed it was safe to recommend PA, oncologists were neutral when it came to their beliefs that patients were capable of achieving activity $(64.4 \%, \mathrm{M}=4.8, \mathrm{SD}=1.3)$.

Another study showed similar results. With a much smaller sample size ( $n=18), 72 \%$ of the physicians, in this case practicing physicians at the Ottawa Hospital Cancer Center, believed that there was sufficient empirical evidence to support the physical benefits of exercise (Peeters et al., 2009). In terms of psychological benefits, an even higher percentage of oncologists, 82\%, felt that sufficient empirical evidence existed to support the use of PA to increase mental health. The low sample size and the fact that all oncologists for this study came from one cancer center has to be considered when examining these results.

These two studies, especially the large scale investigation by Jones et al. (2005) may indicate that oncologists view PA positively and believe that it can be helpful for their patients. Most importantly, they view it as safe. It is interesting that while most of the oncologists studied viewed PA as safe, they did not necessarily believe that their patients were capable of being active. Since oncologists seem to view PA as positive, one may believe that they are actively promoting it. Unfortunately, that does not appear to be the case.

In the previously mentioned study by Jones et al. (2005) besides surveying oncologists’ perceptions of PA, they also surveyed their current practices of promoting PA. It was found that while the majority of oncologists viewed PA positively, more than two thirds (67.8\%) of those surveyed were recommending PA to less than a third of their patients. On average, the 281 practicing oncologists were recommending PA to $28 \%$ of their patients and were spending an 
average of 5.1 minutes having these conversations ( $\mathrm{SD}=3.8)$. These numbers may highlight a need for further education about the promotion of PA; even though the majority of physicians surveyed felt that PA was beneficial, safe, and important, most were not recommending it to a large number of patients and if they did recommend it there were spending little time doing so.

A British study by Daley, Bowden, Rea, Billingham and Carmichael (2008) also sought to learn more about the percentage of physicians recommending exercise. Using a sample of 102 clinicians, 44.1\% $(n=45)$ routinely gave advice to their patients about PA. Using qualitative analyses, it was found that this advice was structured around five themes: benefits for recurrence and mortality; benefits for weight control/management; benefits for physical and functional health; benefits of activity living; and general comments about PA prescriptions (Dailey et al., 2008, p. 47). This sample was made up of oncologists $(n=62,60.8 \%)$ and surgeons $(n=40$, 39.2\%) and it was found that the oncologists were significantly more likely to bring up exercise than their surgical counterparts $(p<0.01)$. While this study provides information about the percentage of British clinicians recommending exercise, there was no information about the quality of this advice. Advice could have simply been briefly mentioning PA. In addition, the low response rate of the study, $14.4 \%$, may increase the chance that there existed a volunteer bias to the study and that the actual percentage of oncologists and surgeons who promote PA is lower than reported here.

Instead of surveying oncologists and their reports of their own behavior, a large study done in Canada surveyed patients and asked if they had discussions with their oncologists about PA (Jones \& Courneya, 2002b). Three hundred and three prostate (30\%), breast (51.8\%), colon/rectum (12.5\%) and lung (5.6\%) cancer patients were surveyed about their discussions of PA with their oncologist. Over fifty percent of patients (57.8\%) reported that they did not have 
any discussions with their oncologist about PA. For those who did, 13.9\% brought it up themselves, while oncologists only brought up the topic with $28.4 \%$ of those surveyed. Of those who had a discussion ( $n=125), 13.6 \%$ were referred to a specialist. The retrospective nature of this study has to be kept in mind when interpreting these results.

Though it seems that many oncologists are not discussing PA with their patients, when these conversations are had patients respond and are more active. A 2004 study (Jones, Courneya, Fairy, \& Mackey) used a randomized controlled trial to investigate the effect that oncologists may have. Four hundred and fifty breast cancer patients were randomized between three groups. One group received a recommendation for PA from their oncologist, another received this recommendation plus a referral to an exercise specialist, and the third group received usual care. Participants were then surveyed about their PA levels five weeks after consultation, but were blinded to the purpose of the survey as numerous other behaviors were also surveyed. In addition, the investigators tested for recall by asking patients if exercise had been recommended and if it had was a referral also made; this happened one week after the initial consultation. The majority of individuals (77\%) within exercise recommendation only intervention groups were able to accurately recall whether or not PA was discussed, while only $41 \%$ of those who received a referral were able to recall this information correctly.

The three groups were then all tested against each other to investigate the effects of recommendations (Jones et al., 2004). Individuals who received a recommendation from their oncologist took part in more exercise per week measured using metabolic equivalent (MET) hours than those in the usual care group (mean difference $=3.4,95 \%$ CI 0.7 to $6.1, p=.011$ ). Interestingly, those in the group that also received a referral did not differ from the usual group on any PA measures. Due to the large number of individuals, especially in the PA 
recommendation plus referral group, who were unable to accurately recall whether or not PA was discussed and if a referral was given, secondary analyses were done between participants who could accurately recall this information and those who did not. Those who did correctly recall information partook in greater amounts of total exercise (mean difference $=4.1,95 \%$ CI 1.9 to $6.4, p<0.001)$. This last finding highlights the important role that oncologists can play in raising in PA, as long as they can make sure their recommendation sticks. The authors of the study posited that individuals who received a referral may not have accurately recalled this at a high rate due to the fact that the referral was simply a business card and this may not have matched their view of what constitutes a referral. In addition, as the authors also noted, the number of individuals who were unable to accurately recall information was similar to the numbers from other studies that examined the recall of information from medical appointments.

Previously, a study by Jones and Courneya (2002b) that investigated the percentage of oncologists discussing PA with their patients was examined. In addition to examining this phenomenon, the investigators also assessed patients levels PA and how they related to the discussions of PA. When an oncologist brought up PA, compared to when PA was not discussed, patients were significantly more likely to have: a higher normative belief of exercise; a greater frequency of mild and moderate exercise; and accumulated more minutes of mild and moderate exercise, as well as total minutes. These findings, along with the findings of Jones et al. (2004) study point to the influential effect that oncologist may have, assuming that they are able to get the information to stick with patients. If the goal is to increase exercise, which as will be discussed below, patients may desire, it is important that oncologists know the most effective to discuss PA so that it "sticks" with their patients. 
To better understand how to best tailor PA interventions for cancer patients, numerous studies have looked at patients' attitudes towards and preferences for PA. As part of the before mentioned study (Jones \& Courneya, 2002b), Jones and Courneya (2002a) investigated individuals’ preferences for both exercise programming and counseling. They also assessed activity levels of participants before they started the intervention described above (Jones \& Courneya, 2002b) and found that just 16\% of the 299 individuals surveyed were meeting ACSM guidelines (Jones \& Courneya, 2002a). Furthermore, only 32\% reported engaging in at least one weekly bout of moderate to strenuous activity. In terms of exercise counseling, 84\% reported that they preferred, or maybe preferred, to receive exercise counseling sometime during their cancer experience. Furthermore, $85 \%$ wanted this to be face to face. In terms of activities, $81 \%$ preferred walking. The most cited location for exercise was home (39.8\%). This study provides firsthand data about the types of interventions that may be preferred by many cancer patients. Overwhelmingly, patients were open to exercise, though they wanted it to be moderate and preferred to do it at home. Their desire to engage in walking as the modality of activity represents an easy behavior for oncologists to promote.

Another study that was discussed earlier also included information about patients’ preferences (Peeters et al., 2009). In this smaller study which had 66 patients, 77\% reported that they would attend an exercise consultation at the hospital during treatment. However, the physicians reported that on average, only $16.7 \%(\mathrm{SD}=11)$ of their patients request information about exercise during treatment. This discourse is interesting; though patients may be potentially open to exercise consultations, often they do not actively seek information on their own. There could be numerous reasons for this. Patients may believe that they are not capable of activity. In addition, the stress of appointments and treatment may lead many to forget about topics of 
interest such as PA to bring up with oncologist. Either way, it is promising that once again a high percentage of individuals surveyed would be open to receiving information about exercise.

Lastly, in a descriptive study of 603 women diagnosed with breast cancer in the American Mid-west, activity rates and exercise preferences were studied (Rogers et al., 2008). These researchers found that patients preferred face to face counseling (61\%), home was the most popular location (36\%), and the vast majority preferred the activity to be at a low or moderate rate (74\%). Most also indicated that they thought they would be able to be moderately active (64\%). However, only 38\% said they would be interested in an exercise program, though another 36\% did say maybe. This large group of individuals who indicated that they might be interested in an exercise program may represent patients who could be potentially swayed by the influence of the oncologist. For most patients, their oncologist and medical staff they are working with are the only sources of information about what they should and should not be doing. There are no large scale media campaigns, and there has not been much coverage in the media about PA for cancer patients so without information from their medical professionals, patients may not think to be active. The previous studies that were examined showed that professionals, especially oncologists, can be positive agents of change points and play an important role of informational gate-keeper with this population.

\section{Appalachian Health Disparities}

The region of the eastern United States that is commonly known as Appalachia has, throughout much of the U.S.'s history, been home to a disportionate number of individuals living under the poverty line that are often at an elevated risk for many health issues (Hartley, 2004). Though there is often some discourse on the exact boundaries of the region, most researchers and other health professionals consider the Appalachian region to run from New York to Mississippi. 
The Appalachian Regional Commission (ARC) has updated their boundaries of this region many times. Currently the ARC defines Appalachian Region as all of West Virginia and parts of 12 other states: Alabama, Georgia, Kentucky, Maryland, Mississippi, New York, North Carolina, Ohio, Pennsylvania, South Carolina, Tennessee and Virginia. This greater than 200 thousand square mile region is home to more than 25 million people (ARC, 2012). The individuals that make up this population, who are mostly white and poor, make less than most other groups of persons and are less likely to have completed high school (Murray, Kulkarni \& Ezzati, 2005).

For decades this group of Americans has been more likely to develop any number of disease states. Though these disparities have existed for much of American history, it is only more recently (past few decades with a significant increase in the 1990s) that researchers have turned their work towards this vulnerable group and only recently have interventions been a strong point of emphasis (Flaskerud, et al., 2002). Appalachian residents have a poorer health status than there non-Appalachian neighbors; this holds true even within states that are only partially within Appalachia (McGarvey, Leon-Verdin, Killos, Guterbock \& Cohn, 2011). However, residents within Appalachia often do not share this view. In a large study of Appalachian adults and their health behaviors it was found that many adults would report that they considered themselves to be "healthy” even if they were sedentary (65\%), hypertensive (76\%), overweight (73\%), or hyperlipidemic (79\%). Additionally, nearly 61\% (+/- 4\%) of individuals who reported themselves as "healthy” possessed at least two disease conditions or poor health behaviors (Griffith, Lovett, Pyle \& Miller, 2011). This distorted view, which may be mediated by the region's lack of education, has been postulated to be one of a myriad of reasons why this area of the country suffers in health (Flaskerud et al., 2002). Other potential contributors include lack of health/prescription services, poor access to health services, a dearth 
of screening services, and poor preventive behaviors and/or services (Huttlinger, Schaller-Ayers \& Lawson, 2004).

Among the health conditions that this population is at an elevated risk for is cancer. With the increase in the rigor of data collection means, researchers have been able to investigate the rates of cancer incidences within Appalachia whereas before much of the region was unable to report reliable numbers. As a whole the Appalachian region is found to have a higher cancer rate than the rest of the nation; central Appalachia (mainly Kentucky and Tennessee) had the highest rates of lung cancer while Southern Appalachia (North and South Carolina, Georgia, Alabama, and Mississippi) and the lowest overall rates in the region (Wingo et al., 2007). Lung cancer specifically affects this region at a much higher rate than the rest of the country. Additionally, cancer patients within Appalachia have been found to be more likely to have higher stage cancers which may be indicative of the lack of health care services (Lengerich et al., 2005). For years this portion of the nation has been underserved in terms of health services and has routinely and predictably suffered because of this. Even with the advent of increased research focused on screening and prevention Appalachian residents still represent one of the U.S.'s most at risk populations for cancer (Murray et al., 2005).

\section{The Concept Mapping Process: Examples from Various Fields of Public Health}

Concept mapping is a term that is often used to describe any process that is aimed at visually representing thoughts, ideas, or relationships. The articles reviewed below represent a specific method of concept mapping developed by Trochim and later refined with Kane (Kane \& Trochim, 2007). This specific concept mapping procedure utilizes a set methodology to gain insight from multiple stakeholders. It is a mixed-method procedure (Greene, Caracelli, \& 
Graham, 1989) that aims to quantize qualitative data to visually represent ideas via two dimensional plot points on a “map.”

Briefly, this concept mapping process consists of six designated steps: preparing for concept mapping, generating the ideas, structuring the statements, analysis, interpretation, and utilization (Kane \& Trochim, 2007). The first step, preparation is similar to the beginning stages of any research or evaluation project. A focus is developed, participants are identified, and logistics such as cost and scheduling are worked through. The next stage, generating the ideas, is when the concept mapping "process" begins. The data that is used within this procedure comes in the form of statements created by stakeholders. This is the stage when these data are created. Stakeholders are often given a question or an open-ended statement to complete. The statements, ideas, or phrases that they develop are then in turn the data. For instance, in one study aimed at identifying ways that tobacco companies in South East Asia try to block tobacco control, the research team had stakeholders complete the following sentence: “A specific activity that the tobacco industry uses to block tobacco control in South East Asia is” (Stillman, Hoang, Linton, Ritthiphakdee, \& Trochim, 2008, p 2). The activities that the stakeholders came up with were then the data that were used in subsequent steps to generate the visual maps. This process of statement generation is often done online, or can be done in group settings similar to focus groups. Members of the research team condense the statements across stakeholders to reduce redundancy. In addition when large numbers of stakeholders are utilized or when the process is trying to be done quickly, stakeholders may be restricted in the number of statements they can produce (e.g. Anderson et al., 2006).

Once the statements have been finalized, stakeholders next sort the statements and rate them (Kane and Trochim, 2007). Again, this can be done in person or through web based 
software. Stakeholders are instructed to sort the statements into groups as they see fit. They are to create more than one group and that each statement cannot be its own group. In person, this is often achieved by printing out the statements on note cards and having individuals create piles of note cards that they believe are related. In addition to sorting statements it is during this step that additional data can be collected. Statements can be rated on measures determined valuable by the research team. For instance, in the above example of tobacco control in South East Asia stakeholders rated each statement on importance and feasibility of collecting data (Stillman, et al., 2008). The next step, analysis, is when quantitative statistics come into the equation. First, multidimensional scaling is used to give each statement two-dimensional $(x, y)$ coordinates. Each statement is "plotted" on a two-dimensional map using these coordinates. Statements that are close geographically on the map were judged to be similar by stakeholders. The coordinates in and of themselves are arbitrary as they are created to "measure" distance; the map could be rotated or the coordinates switched ( $x$ to $y, y$ to $x$ ) without any effect, other than visual contortion, on the outcome. Hierarchical cluster analysis is next done to group statements. This process divides the statements into groups starting with one group and ending with each statement representing a group. There is no singular way to determine how many clusters there should be. Often stakeholders are used to judge the "correct” number of clusters. Individual statements can be identified as bridging or anchor statements. A bridging statement is one that will go from one group to another when the number of clusters is increased. These statements can be valuable as they can provide insight into when clusters becomes too diverse or conversely when there is not enough groups to adequately divide the statements into unique groupings. Anchor statements are statements that many stakeholders believed were related to surrounding statements. These statements often represent other statements that are near which can be helpful 
when determining if the groupings of statements logically belong together. In addition to the tests done to determine the number of groupings and placement of statements, multiple analyses can be done on the rating data of the statements (examples of such analyses will be highlighted below when appropriate).

The last two steps, interpretation and utilization are often done once again with stakeholders. Stakeholders are provided with the maps and other data from the analysis steps. During this stage the groups are often labeled. In addition, other relationships may be highlighted such as larger groupings of clusters. The last step varies significantly depending on the original goal of the project. For many concept mapping projects the goal is evaluative in nature, often developing evaluation framework. This framework is then developed and put into practice at the completion of the concept mapping process.

A 2006 article by Anderson and colleagues highlights the use of concept mapping to gather information from a geographically diverse set of stakeholders. The US Center for Disease Control (CDC) has developed a network of 33 Prevention Research Centers throughout the country. These centers represent the largest extramural research network of the CDC. The researchers sought to help the CDC gather input from the different Prevention Research Centers to develop a logic model that would serve as the foundation for an evaluation framework as well as begin to determine program expectations. The concept mapping process was utilized to get input from stakeholders across the country. Maps were created to produce logic models at both the national and community level. One hundred and seventy five stakeholders were identified for the national model while 165 were identified for the local model. Members either participated online or through the mailing/faxing of documents. Individuals were told to submit up to 10 ideas in response to the following prompts: "To ensure national excellence in 
prevention, a Prevention Research Center should have the following characteristic or function” (p3) and "To successfully promote health in a community, an effective Prevention Research Center Should have the following specific characteristic or skill” (p3). It was estimated that 145 (83\%) and 135 (82\%) national and local stakeholders respectively provided statements. The final national statement list contained 88 unique statements while the local list contained 75.

Once the statements had been collected, smaller subsets of both national $(n=35)$ and local $(n=30)$ stakeholders sorted the data (Anderson et al., 2006). In both cases, 57\% $(n=20, n$ $=17$ for the national and local levels respectively) of participants completed this step. The 88 statements for the national map were grouped into nine clusters while the local map created 11 clusters from 75 statements. The resulting clusters when then used as the foundation for logic models that were then sent to stakeholders for feedback. Stakeholders suggested slight revisions to the model and the logic models are now utilized by the CDC to evaluate the effectiveness of Prevention Research Centers.

This article is an excellent example of the usefulness of the concept mapping procedure to assess a network of shareholders (Anderson et al., 2006). In addition, this study is an example of having stakeholders design evaluative framework that they will then be evaluated on. This process can help ensure that all parties agree on the evaluation methods and that the methods truly reflect the beliefs of those being evaluated. The relatively modest number of individuals who completed (57\% for both the national and local groups) the sorting step of the process is somewhat surprising and highlights a potential limitation of this approach. A recent project completed by Macdiarmid and colleagues also set out to develop an evaluative framework (2010). In this case, the research team was interested in developing an evaluative framework for nutrition-related policy interventions. In addition, they wanted this framework to include a 
timeline of when changes could be expected. To develop this framework, a specific policy intervention, the Schools (Health Promotion and Nutrition) Act of 2007 out of Scotland was used to serve as the policy that would be evaluated. Stakeholders were asked to complete the following sentence: "I think the introduction of the Schools (Health Promotion and Nutrition) (Scotland) Act 2007 in primary schools will” (Macdiarmid et al., 2010, p2). Stakeholders were recruited from national and local government, community organizations, schools, and relevant scientific research communities. A total of at least 291 individuals were contacted (the actual number may be larger as certain organizations forwarded an email request for participation that the research team was unable to track).

Statements were generated based on the above mentioned prompt either through interviews, a workshop at a conference, or focus-groups (Macdiarmid et al., 2010). A total of 40 individuals participated. Over 300 statements were created, which was condensed to 85 by the research team. To sort and rate the data a larger number of stakeholders (the number was not supplied by the researchers) were contacted, of which 102 completed all the necessary steps. Individuals not only sorted the data as they saw fit, but they also rated each item on its likelihood of occurring, the importance of the outcome, as well as the earliest anticipated time to measurable impact. The likelihood and importance data was collected using 5-pt Likert-type scales while the time to impact was measured using 7-pt Likert-type scales ( $0=$ immediate, to 6 $=6$ or more years).

Thirteen clusters were created based on the work of these stakeholders (Macdiarmid et al., 2010). Examples of the clusters include: "Promote Well-Being”, “Increase Food Literacy”, and “Encourage Healthy Diet Choices”. When clusters' items were examined based on the ratings, “Increase Food Literacy” was seen as the most important, as well as the cluster rated 
most likely to occur. Interestingly, in terms of time until measurable impact, "Adverse Economic Consequences” was rated as the cluster which could be measured the soonest while logically, “Long-Term Health Gains and Impact” was thought to be last to be able to have its impact measured.

Though the actual response rate for the sorting and rating stage of this project was not reported, the fact that 102 individuals completed all steps is promising and may signal that with proper planning and proper identification, stakeholders may be willing to spend the time necessary to complete this time consuming task.

Besides evaluation, another task that concept mapping is often utilized for is planning. In 2004 Trochim and colleagues used concept mapping to help the state of Hawaii’s health department better understand the community and system factors that affect individuals’ tobacco, nutrition, and physical activity behaviors (Trochim, Milstein, Wood, Jackson, \& Pressler 2004). The state of Hawaii was interested in this line of inquiry so that it could better understand how to spend the money that was allocated to the state as part of the tobacco industries' settlement with the U.S. Attorneys General in 1998-approximately $\$ 1.3$ billion, which the state want to spend mostly on public health concerns. To learn more about the factors related to the state's health, Trochim and colleagues (2004) asked stakeholders, including 34 Hawaiian health professionals as well as 46 national experts, to "Generate statements that describe specific community or systems factors that affect individuals’ behaviors related to tobacco, nutrition, and physical activity” (p 11).

Participants provided over 400 statements either online through a web-based system or through in-person brainstorming sessions (Trochim et al., 2004). These data were then condensed into a final set of 90 unique statements. For the next step, in addition to sorting the 
statements into groups, participants $(n=25)$ using five-point Likert-type scales rated each statement on importance and feasibility. The 90 statements were grouped into seven clusters: policies and laws; environment infrastructure; access; information/communication; community infrastructure; coalitions/collaborations; and children and school. Among these clusters, environment infrastructure, policies and laws, and community infrastructure were judged to be the most important while policies and laws, community infrastructure, and information/communication were thought to be the most feasible for achieving change in the near future.

At the conclusion of the concept mapping process, the results were used to develop the Healthy Hawaii Initiative that was the state legislative act that outlined how the settlement money would be spent (Trochim et al., 2004). This project highlights pragmatic reasons to use the concept mapping process. Feedback was needed from a number of stakeholders, both local and national. In addition, many of these stakeholders would be the very health professionals carrying out any policy changes or health initiatives so their input was extremely critical. The state also needed this information fast as to make sure it would be ready for review by various government officials. The concept mapping process was completed in eight business days. This quick turnaround between stages may explain while less than half of the original stakeholders completed the sorting tasks. The online system was only open for two days. A longer duration may have led to a higher percentage of stakeholders completing this crucial step. Even with the decreased number of individuals who sorted and rate the data, this study highlights the usefulness of concept mapping and the potentially powerful tool that it can be.

Trochim and colleagues 2004 work is not the only example of concept mapping within a tobacco context. While in the U.S. tobacco use has steadily declined, in other areas of the world 
the opposite is true. South East Asia is one example of an area of the world where tobacco use is on the rise. To better understand potential reasons behind this rise, researchers utilized concept mapping to develop a systematic approach to track and monitor tobacco industry activities both locally and regionally within four countries: Cambodia, Malaysia, Thailand, and Vietnam (Stillman et al., 2008). Participants for this project were members of the South East Asia Tobacco Control Alliance. Both the statement generating brainstorming sessions and the sorting sessions took place in person in each of the four countries or at an international conference. A total of 35 individuals helped generate statements while 31 individuals sorted and rated data.

The statement used to elicit statements from stakeholders was: “A specific activity that the tobacco industry uses to block tobacco control in South East Asia is:” (Stillman et al., 2008, p. 2). A final set of 86 statements was conceived from this step. In addition to sorting the statements, participants rated each item for importance as well as the feasibility of collecting or obtaining data related to the statement on five-point Likert-type scales. Eight clusters were created, of which “Marketing Tactics and Image Building” was the deemed the most important and had the highest feasibility rating for collecting data. The clusters were then further grouped into four sectors: economics, politics, deception, and public relations.

The findings of this study could aid tobacco control in South East Asia in a number of ways (Stillman et al., 2008). Measurements could be created utilized the cluster as areas of focus. In addition, the ratings and the subsequent analyses could be used to help target areas for intervention. By examining not only the importance ratings of clusters/items the feasibility scores can be used to highlight where change may be seen first. The goal of this project was to develop a systematic approach to track and monitor the tobacco industry in South East Asia, this 
was utilized by highlighting the ways that the industry blocks or avoids control through the use of the concept mapping procedures.

Besides planning and evaluation, concept mapping can also be used to begin to develop theories by better understanding phenomena. Two examples of this took place in very different domains: the role of neighborhood factors in intimate partner domestic violence (Burke, Campo, Peak, Gielen, McDonnell, \& Trochim, 2005) and supported employment for individuals with mental illness (Trochim, Cook, \& Setze, 1994). In both cased stakeholders who had personal knowledge and/or experience of the subject manner were utilized. In the first example, women from Baltimore City who had participated in previous research projects related to intimate partner violence were recruited. This recruitment technique resulted in 14 women who took place in live brainstorming sessions and an additional 23 women who helped sort and rate the data, once again in live in-person group sessions (Burke et al., 2005). In the example examining supported employment for individuals with mental illness, employees of a mental health facility that worked closely with the supported employment services were utilized ( $n=14$; Trochim et al., 1994).

In Burke and colleagues’ project (2005), the women in the study created 46 unique statements that were then divided among seven groups. In addition, each statement was rated on five-point Likert-type scales for: prevalence (strength of perceived relationship between item and intimate partner violence), severity (degree to which item worsens severity of intimate partner violence), perpetration (relationship of item to a man’s perpetration of intimate partner violence), and cessation (degree to which item supports cessation of intimate partner violence). In Trochim, Cook, and Setze’s project (1994), mental health employees working with supportive 
employment developed 18 clusters based on 96 statements; each item was also rated on importance with a five-point Likert-type scale.

Both of these projects (Burke et al., 2005; Trochim et al., 1994) highlight the ability of concept mapping to begin to inform theory. Since neither of the subject areas is pertinent to this review, further exploration of potential for theory development within each area was not warranted. Both projects used stakeholders that were knowledgeable of the subject areas through personal experience. This strengthens the real world applicability of the findings of both projects. National academic experts are often valuable participants for concept mapping studies as many studies described above indicated; however, often it can be useful to get firsthand knowledge. Concept mapping can be one such tool to gather this knowledge in a uniform and structured manner. In both Burke et al.’s (2005) and Trochim et al.’s (1994) projects, participants responded positively and in Burke’s project participants inquired about volunteering further with similar projects. Though the process can be very involved and time consuming for participants, these findings help to strengthen the expectation that this process is manageable and the process often provides something that many research projects do not - a tangible, and potentially meaningful outcome for participants.

These projects taken together show the usefulness of concept mapping within various health-related fields to gather input from large numbers of stakeholders. Concept mapping can also allow for researchers to gather information from individuals who are geographically spread out, this is especially true with the advent of online based software for running concept mapping projects. Though there are many positives to the process and the results can be very useful, the process itself is time consuming and maintaining participation can be a challenge, especially 
when there are a large number of statements created. Nonetheless, concept mapping could be put to good use in many domains related to public health.

\section{Conclusion and Future Recommendations}

Though PA has received considerable attention throughout the past decade much work is still needed. Previous work has been of mixed methodological rigor and findings have been inconsistent. The recent trend of randomized controlled trials has been a positive step in the right direction. However, study outcomes have varied widely and more focused attention may be needed on specific outcomes.

A current weakness of the field is a lack of understanding about what outcomes are needed for oncologists to more widely promote PA. It seems that exercise being safe, tolerable, and desired by patients is not enough. The current findings that increased PA may also be linked to higher survival rates and lower reoccurrences rates have also not driven a change in standard cancer treatment. One of the weaknesses that permeates this line of research and may be keeping exercise from being included in standard cancer care is the lack of understanding about the doseresponse relationship between exercise and various outcomes. Novel methods have been employed in recent studies to try to control for various effects and it is time for methods to be used to compare outcomes based on levels of exercise. In relation to better understanding the frequency, intensity, and duration of exercises best suited for potential positive outcomes, patients' perspectives need to be explored as well. The positive effects of exercise during or after treatment may not be completely due to physical changes. There may be emotional, psychological, and motivational changes that patients experience. To better understand these possible relationships, patients' perceptions and experiences need to be explored and this 
potential avenue represents an area where qualitative procedures could be used to add depth and even target areas for quantitative investigation.

One way to address the above mentioned weakness of a lack of understanding of oncologists' perceptions of what needs to happen for PA to be more actively promoted might be to simply ask. Oncologists represent a logical but underused population for investigation. Current behaviors related to the promotion of exercise and perceptions of its usefulness have been explored, but it does not seem that the suggestions of the very individuals who could serve as powerful catalysts for change With regard to cancer survivors' health behaviors have been sought. If the research community within the realm of PA and cancer treatment better understood what the oncologists desired, they could shape their research to better meet the oncologists needs which could lead to changes in practice. Concept mapping could be a means of gathering input from not only oncologists but also from researchers. The findings of such a study could help researchers better tailor their investigations. In addition, ideas for improved dissemination of research findings may be realized. Oncologists could better understand the barriers that researchers perceive and can work to lessen these barriers. Guidelines or suggestions for not only research but also for practice could be developed with input from both the research and the practice camps. Research in this field has and will most likely continue to grow. In addition, the number of cancer survivors will continue to grow. If the goal of research is to inform practice and identify ways to improve the QOL of patients, then the research community would benefit greatly from better understanding the needs of the practicing medical community. And if the goals of oncologists are to promote better health in their patients then a better understanding of how to incorporate research findings would be of great benefit as well. 


\section{References}

Adamsen, L., Quist, M., Andersen, C., Moller, T., Herrstedt, J., Kronborg., . . Rorth, M. (2009). Effect of a multimodal high intensity exercise intervention in cancer patients undergoing chemotherapy: Randomized controlled trial. British Medical Journal, 339, 895-899.

Airley, R. (2009). Cancer chemotherapy: Basic science to the clinic. Chichester, West Sissex, UK: Wiley-Blackwell.

Anderson, L.A., Gwaltney, M.K., Sundra, D.L., Brownson, R.C., Kane, M, Cross, A.W., .. . White, C.R. (2006). Using concept mapping to develop a logic model for the prevention research centers program. Preventing Chronic Disease, 3, 1-09.

Appalachian Regional Commission. (2012). Appalachian regional commission homepage. Retrieved from: http://www.arc.gov/

Burke, J.G., O’Campo, P., Peak, G.L., Gielen, A.C., McDonnell, K.A., \& Trochim, W.M.K. (2005). An introduction to concept mapping as a participatory public health research method. Qualitative Health Research, 15, 1392-1410.

Courneya, K., \& Friedenreich, C. (2001). Framework PEACE: An organizational model for examining physical exercise across the cancer experience. Annals of Behavioral Medicine, 23, 263-272

Courneya, K.S., Segal, R.J., Mackey, J.R., Gelmon, K., Reid, R.D., Friedenreich, C.M., .. . McKenzie, D.C. (2007). Effects of aerobic and resistance exercise in breast cancer patients receiving adjuvant chemotherapy: A multicenter randomized controlled trial. Journal of Clinical Oncology, 25, 4396-4404.

Daley, A.J., Bowden, S.J., Rea, D.W., Billingham, L. \& Carmicheal, A.R. (2008). What advice are oncologists and surgeons in the United Kingdom giving to breast cancer patients 
about physical activity? International Journal of Behavioral Nutrition and Physical Activity, 5, 46. Online publication.

Daley, A.J., Crank, H., Saxton, J.M., Mutrie, N., Coleman, R. \& Roalfe, A. (2007). Randomized trial of exercise therapy in women treated for breast cancer. Journal of Clinical Oncology, 13, 1713-1721.

Flaskerud, J.H., Lesser, J., Dixon, E., Anderson, N., Conde, F., Kim, S., . . Verzemnieks, I. (2002). Health disparities among vulnerable populations. Nursing Research, 2, 74-85.

Goodwin, P.J., Ennis, M., Pritchard, K.I., Trudeau, M.E., Koo, J., Madarnas, Y., ... Hood, N. (2002). Fasting insulin and outcome in early-stage breast cancer: Results of a prospective cohort study. Journal of Clinical Oncology, 20, 42-21

Greene, J.C., Caracelli, V.J., \& Graham, W.F. (1989). Toward a conceptual framework for mixed-methods evaluation design. Educational Evaluation and Policy Analysis, 11, 225274.

Greenlund, K.J., Giles, W.H., Keenan, N.L., Croft, J.B., Mensah, G.A. \& Huston, S.L. (2002). Physician advice, patient actions, and health-related quality of life in secondary prevention of stroke through diet and exercise: The physician's role in helping patients to increase physical activity and improve eating habits. Stroke, 33, 565-571.

Griffith, B.N., Lovett, G.D., Pyle, D.N. \& Miller, W.C. (2011). Self-rated health in rural Appalachia: Health perceptions are incongruent with health status and health behaviors. BMC Public Health, 11(229), 1-8.

Hartley, D. (2004). Rural health disparities, population health and rural culture. American Journal of Public Health, 94, 1675-1678. 
Holick, C.N., Newcomb, P.A., Trentham-Dietz, A, Titus-Ernstoff, L., Bersch, A.J., Stampfer, M.J., . . . Willett, W.C. (2008). Physical activity and survival after diagnosis of invasive breast cancer. Cancer Epidemiological Biomarkers Prevention, 17, 379-386.

Holmes, M.D., Chen, W.Y., Feskanich, D., Kroenke, C.H., \& Colditz, G.A. (2005). Physical activity and survival after breast cancer diagnosis. JAMA, 293, 2479-2486.

Huttlinger, K., Schaller-Ayers, J, \& Lawson, T. (2004). Health care in Appalachia: A populationbased approach. Public Health Nursing, 21, 103-110.

Jones, L.W. \& Courneya, K.S. (2002a). Exercise counseling and programming preferences of cancer survivors. Cancer Practice, 10, 208-215.

Jones L.W. \& Courneya, K.S. (2002b). Exercise discussions during cancer treatment consultations. Cancer Practice, 10, 66-74.

Jones, L.W., Courneya, K.S., Fairy, A.S. \& Mackey, J.R. (2004). Effects of an oncologist’s recommendation to exercise on self-reported exercise behavior in newly diagnosed breast cancer survivors: A single-blind, randomized controlled trial. Annals of Behavioral Medicine, 28, 105-113.

Jones, L.W., Courneya, K.S., Peddle, C. \& Mackey, J.R. (2005). Oncologists’ opinions towards recommending exercise to patients with cancer: a Canadian national survey. Supportive Cancer Care, 13, 929-937.

Kane, M. \& Trochim, W.M.K. (2007). Concept mapping for planning and evaluation. Thousand Oaks, CA: Sage.

Lengerich, E.J., Tucker, T.C., Powell, R.K., Colsher, P., Lehman, E, Ward, A.J., .. W Wyatt, S.W. (2005). Cancer incidence in Kentucky, Pennsylvania, and West Virginia: Disparities in Appalachia. The Journal of Rural Health, 21, 39-47. 
Ligibel, J.A., Campbell, N., Partidge, A., Chen, W.Y., Salinardi, T., Chen, H., . . Winer, E.P. (2008). Impact of a mixed strength and endurance exercise intervention on insulin levels in breast cancer survivors. Journal of Clinical Oncology, 26, 907-912.

Loureiro, M.L. \& Nayga, R.M. (2006). Obesity, weight loss, and physician’s advice. Social Science and Medicine, 62, 2458-2468.

Lowitz, B.B. \& Casciato, D.A. (2009). Principles, definitions, and statistics. In D.A. Casciato \& M.C. Territo (Eds), Manual of clinical oncology (3-18).

Macdiarmid, J.I., Loe, J., Douglas, F., Ludbrook, A., Comerford, C., \& McNeill, G. (2010). Developing a timeline for evaluating public heath nutrition policy interventions. What are the outcomes and when should we expect to see them? Public Health Nutrition, 1, 1-11.

Matthews, C.E., Wilcox, S., Hanby, C.L., Der Ananian, C.D., Heiney, S.P., Gebretsadik, T. \& Shintani, A. (2007). Evaluation of a 12-week home-based walking intervention for breast cancer survivors. Supportive Care Cancer, 15, 203-211.

McBride, C.M., Clipp, E., Peterson, B.L., Lipkus, I.M., \& Demark-Wahnefried, W. (2000). Psychological impact of diagnosis and risk reduction among cancer survivors. PsychoOncology, 9, 418-427.

McGarvey, E.L., Leopn, Verdin, M., Killos, L.F., Guterbock, T. \& Cohn, W.F. (2011). Health disparities between Appalachian and non-Appalachian counties in Virginia USA. Journal of Community Health, 36, 348-356.

Milne, H.M., Wallman, K.E., Gordon, S. \& Courneya, K.S. (2008). Effects of a combined aerobic and resistance exercise program in breast cancer survivors: A randomized controlled trial. Breast Cancer Research and Treatment, 108, 279-288. 
Mutrie, N., Campbell, A.M., Whyte, F, McConnachie, A, Emslie, C, Lee, L., . . Ritchie, D. (2007). Benefits of supervised group exercise programme for women being treated for early stage breast cancer: Pragmatic randomized controlled trial. British Medical Journal, 334, 517-524.

Murray, C.J.L., Kulkarni, S. \& Ezzati, M. (2005). Eight Americans: New perspective on U.S. health disparities. American Journal of Preventive Medicine, 29(5S1), 4-10.

National Cancer Institute, (1999). Chemotherapy and you: A guide to self-help during cancer treatment. Retrieved from http://www.cancer.gov/cancertopics/chemotherapy-andyou/page2

Peeters, C., Stewart, A., Segal, R., Wouterloot, E., Scott, C.G., \& Aubry, T. (2009). Evaluation of a caner exercise program: Patient and physician beliefs. Psycho-Oncology, 18, 898902.

Pinto, B.M., Rabin,C. \& Dunsiger, Shira. (2009). Home-based exercise among cancer survivors: Adherence and its predictors. Psycho-Oncology, 18, 369-376.

Pub Med. (2011). Lymphatic obstruction. Retrieved from: http://www.ncbi.nlm.nih.gov/pubmedhealth/PMH0002106/

Rogers, L.Q., Courneya, K.S., Verhulst, S., Markwell, S., Lanzotti, V. \& Shah, P. (2008). Exercise barrier and task self-efficacy in breast cancer patients during treatment. Supportive Cancer Care, 14, 84-90.

Schmitz, K.H, Courneya, K.S., Matthews, C., Demark-Wahnefried, W., Galvao, D.A., Pinto, B.M., . . . Schwartz, A.L. (2010). American college of sports medicine roundtable on exercise guidelines for cancer survivors. Medicine and Science in Sports and Exercise, 42, 1409-1426. 
Segal, R.J., Reid, R.D., Courneya, K.S., Sigal, R.J., Kenny, G.P., Prud’Homme, D.G., . . . Slovinec D’Angelo, M.E. (2009). Randomized controlled trial of resistance or aerobic exercise in men receiving radiation therapy for prostate cancer. Journal of Clinical Oncology, 27,344- 351.

Speck, R.M., Courneya, K.S., Masse, L.C., Duval, S. \& Schmitz, K.H. (2010). An update of controlled physical activity trials in cancer survivors: a systematic review and metaanalysis. Journal of Cancer Survivorship, 4, 87-100.

Sternfeld, B., Weltzien, E., Quesenberry, C.P., Castiollo, A.L., Kwan, M., Slattery, M.L. \& Caan, B.J. (2009). Physical activity and risk of recurrence and mortality in breast cancer survivors: Findings from the LACE study. Cancer Epidemiological Biomarkers Prevention, 18, 87-95.

Stillman, F. Hoang, M., Linton, B., Ritthiphakdee, B. \& Trochim, W. (2008). Mapping tobacco industry strategies in south east Asia for action planning and surveillance. Tobacco Control, 17, 1-7. Online publication.

Trochim, W.M.K., Milstein, B., Wood, B.J., Jackson, S., \& Pressler, V. (2004). Setting objectives for community and systems change: An application of concept mapping for planning a statewide health improvement initiative. Health Promotion Practice, 5, 8-19.

Trochim, W.M.K., Cook, J.A., \& Setze R.J. (1994). Using concept mapping to develop a conceptual framework of staff's views on a supported employment program for individuals with severe mental illness. Journal of Counseling and Clinical Psychology, 62, 766-775.

Wingo, P.A. Tucker, T.C., Jamison, P.M., Martin, H., McLaughlin, C., Bayakly, R., .. . Richards, T.B. (2007). Cancer in Appalachia, 2001 - 2003. Cancer, 112, 181-192. 